\title{
APPEARANCE AND SPECTROSCOPIC CHARACTERIZATION OF AUTOMOTIVE COATINGS FOR FORENSIC PURPOSES
}

\author{
A Thesis \\ presented to \\ the Faculty of California Polytechnic State University, \\ San Luis Obispo

\begin{abstract}
In Partial Fulfillment
of the Requirements for the Degree

Master of Science in Polymers and Coatings Science
\end{abstract}

by

Kimberly Kha

December 2017 
(C) 2017

Kimberly Kha

ALL RIGHTS RESERVED 
COMMITTEE MEMBERSHIP

TITLE: $\quad$ Appearance and Spectroscopic

Characterization of Automotive Coatings

for Forensic Purposes

AUTHOR: Kimberly Kha

DATE SUBMITTED: December 2017

COMMITTEE CHAIR: Raymond Fernando, Ph.D.

Professor of Chemistry, Director of

Polymers and Coatings Program

COMMITTEE MEMBER: Stephanie Watson, Ph.D.

Polymeric Materials Group

National Institute of Standards and

Technology

COMMITTEE MEMBER: $\quad$ Erik Sapper, Ph.D.

Assistant Professor of Chemistry 


\author{
ABSTRACT \\ Appearance and Spectroscopic Characterization of \\ Automotive Coatings for Forensic Purposes \\ Kimberly Kha
}

Automotive paint is a common type of trace evidence in the forensics community due to the prevalence of vehicular collisions. Forensic examinations of these samples typically involve visual inspection for physical properties such as color. However, these observations are subject to variability due to observer and environmental factors. Therefore, objective methods of color description based on spectrophotometry using coordinate systems are now recommended by US forensics standards in addition to chemical analysis for binder and pigment information. Color meters and microspectrophotometers (MSP) are common instruments for quantifying color descriptions using opponent coordinate systems such as the International Commission on Illumination CIEL ${ }^{*} \mathrm{a}^{*} \mathrm{~b}^{*}$ color space (CIELAB). MSP is used on small fragments encountered in forensic cases, but requires extensive sample preparation and expensive instrumentation while color meters are frequently employed by coating manufacturers and portable for field use. The two methods were compared by calculating color difference, $\mathrm{DE}^{\star}$, between fifteen automotive coating samples and standards to gauge differences and associated errors. A correlation between color meter and reflectance-mode MSP $\mathrm{DE}^{*}$ was found. After physical attributes are noted in forensic analyses, samples are often characterized by molecular spectroscopy. IR and Raman spectroscopic analyses 
were performed on the sample set to determine their utility in binder and pigment identification. Binder chemistries of all layers in the set were classified by IR spectroscopy, and Colour Index (Cl) pigments in the base coats were identified by Raman spectroscopy. Finally, as UV exposure can alter the chemistry of automotive coatings over time, controlled artificial weathering of clear coats from two major manufacturers was performed at $55^{\circ} \mathrm{C}$ and $75 \%$ relative humidity $(\mathrm{RH})$ with $100 \%$ ultraviolet (UV) radiation using the SPHERE (Simulated Photodegradation via High Energy Radiant Exposure) at the National Institute of Standards and Technology. Appearance and spectroscopic measurements of the samples were used to track photodegradation. Color and gloss were used as physical measurements, while IR and Raman spectroscopies were applied to quantify general binder degradation, and transmission-MSP was used to track UV absorber concentration. The combination of color measurements by spectrophotometry and chemical characterization by spectroscopy should enhance the objectivity of forensic examinations of trace automotive coating evidence. 


\section{ACKNOWLEDGMENTS}

I would like to thank Dr. Stephanie Watson, Dr. LiPiin Sung, and everyone in the Polymeric Materials Group at the National Institute of Standards and Technology for their guidance during my time there. I would also like to thank my committee, Dr. Raymond Fernando and Dr. Erik Sapper for their advice and support. Finally, I would like to thank the Kenneth N. Edwards Western Coatings Technology Center, and the Polymers and Coatings graduate program. 


\section{CHAPTER}

1. Overview 1

2. Spectrophotometric Color Analysis of Automotive Coatings 4

2.1 Introduction 4

2.2 Experimental 12

$\begin{array}{lll}2.3 & \text { Results and Discussion } & 15\end{array}$

2.4 Conclusions 24

3. Spectroscopic Analysis of Automotive Coatings 25

$\begin{array}{lll}3.1 & \text { Introduction } & 25\end{array}$

3.2 Experimental 31

3.3 Results and Discussion 33

3.4 Conclusions 56

4. Accelerated Weathering of Automotive Clear Coats 58

$\begin{array}{lll}4.1 & \text { Introduction } & 58\end{array}$

4.2 Experimental 69

$\begin{array}{lll}4.3 & \text { Results and Discussion } & 76\end{array}$

$\begin{array}{lll}4.4 & \text { Conclusions } & 90\end{array}$

5. Final Remarks 91

REFERENCES 93

$\begin{array}{ll}\text { APPENDIX } & 102\end{array}$ 


\section{LIST OF TABLES}

Page

Table 2.1 Automotive Coatings Examined 13

Table 2.2 DE* of Sample Pairs from Same Vehicle 17

Table 2.3 DE* of Same Color Metallic Blue Samples Examined 19

Table 2.4 DE* of Flat / Metallic Standard / Sample Pairs 22

Table 2.5 Correlation Coefficients for Color Meter and MSP DE* 23

Table 3.1 Original Finish Binders on Current Vehicles ${ }^{10} \quad 28$

Table 3.2 Binder Chemistry of Clear Coats and Base Coats Identified by IR

Spectroscopy 35

Table 3.3 Binder Chemistries of Primers Identified by IR Spectroscopy 36

Table 3.4 Composition of Substrates Determined by IR Spectroscopy 36

Table 3.5 Raman Spectroscopy Results $\quad 49$

Table 4.1 Properties of Clear Coats Studied 70

Table 4.2 Raman Spectroscopy Parameters for Clear Coats 76

Table 4.3 Degradation Rate Differences between Instruments 82

Table 4.4 Degradation Rate Differences by Color and Chemistry 82

Table 4.5 Rates of Total Clearcoat Absorbance Decline 85 


\section{LIST OF FIGURES}

Page

$\begin{array}{ll}\text { Figure 2.1 Layers comprising a typical automotive coating. } & 6\end{array}$

Figure 2.2 Visualization of CIELAB system including chroma and hue angle. $\quad 7$

Figure 2.3 Illumination-detection geometries recommended by ASTM E2194 for metal flake pigmented materials. ${ }^{24}$

Figure 2.4 General layer sequences observed in the sample set.

Figure 2.5 T-MSP spectra of (a) metallic blue samples 18A (black) and 19A (red), and (b) flat red samples 12 (black) and 13 (red).

Figure 2.6 T-MSP spectra of (a) metallic black samples 15 (black) and 17 (red), and (b) metallic white samples 58 (black) and 59 (red).

Figure 2.7 White samples measured by MSP in reflection and transmission modes.

Figure 2.8 Scatterplot matrix and histograms of CM, R-MSP, and T-MSP DE*, indicating lack of normality.

Figure 3.1 Structures of common binder components in automotive clear and base coats.

Figure 3.2 FTIR spectrum of the refinish clear coat of sample 11, an "oxithanetype" urethane-modified acrylic binder. Arrows represent key peaks.

Figure 3.3 FTIR spectrum of acrylic clear coats with melamine and styrene modifications. Asterisks represent key peaks.

Figure 3.4 FTIR spectra of acrylic, alkyd, and polyester coating layers. Vertical lines delineate the region of interest for differentiation between these binder types; asterisks represent the highest intensity peak in this region. 
Figure 3.5 FTIR spectrum of the epoxy primer of sample $1 \mathrm{~A}$. Arrows represent key peaks for epoxy identification.

Figure 3.6 FTIR spectrum of the substrates of samples 11 (PU) and 13 (PEPP). Asterisks represent key peaks for identification of these substrate chemistries.

Figure 3.7 FTIR spectra of automotive coating layers containing barium sulfate (black), calcium carbonate (blue), titanium dioxide (red), and talc (green). Asterisks represent the key peaks for identifying these additives in IR spectra. 43

Figure 3.8 Score plot for PCs 1 and 2 of clear coat IR spectra set.

Figure 3.9 Factor loadings for PC 1 of the clear coat FTIR spectra set. Arrows represent peaks corresponding to melamine modification.

Figure 3.10 Score plot of PCs 1 and 2 of base coat IR spectra set.

Figure 3.11 Factor loadings for PC 2 of the base coat FTIR spectra set. Arrow represents the peak corresponding to titanium dioxide.

Figure 3.12 Score plots for PCs 1 and 2 of primer IR spectra set.

Figure 3.13 Factor loadings for PC 1 of the primer FTIR spectra set. Arrows represent peaks indicating presence of epoxy.

Figure 3.14 Score plots for PCs 1 and 2 of substrate IR spectra set (left). The cluster on the left represents the substrate of sample 11. Factor loadings for PCs 1 and 2 of the substrate FTIR spectra set (right). The asterisks indicate key peaks corresponding to PU in PC 1 and talc in PC 2.

Figure 3.15 Factor loadings of the first three PCs for clear coat Raman spectra taken with the $532 \mathrm{~nm}$ laser. Asterisks identify the highest intensity peaks in the factor loadings of each PC.

Figure 3.16 Factor loadings of the first three PCs for clear coat Raman spectra taken with the $785 \mathrm{~nm}$ laser. Asterisks identify the highest intensity peaks in the factor loadings of each PC. 
Figure 3.17 Raman spectra of (top) an oxithane acrylic urethane clear coat, (middle) an acrylic styrene clear coat, and (bottom) an acrylic melamine clear coat taken with the $785 \mathrm{~nm}$ laser. Vertical lines identify chemical composition analyzed in the discussion.

Figure 4.1 Effect of relative humidity on photo-oxidation rate of acrylicmelamine coatings. ${ }^{69}$

Figure 4.2 Action spectrum illustrating the effect of wavelength range on quantum efficiency. ${ }^{70}$

Figure 4.3 General scheme of polyester photo-oxidation. ${ }^{71}$

Figure 4.4 Melamine (a) and carbamate (b) crosslink photodegradation reactions. ${ }^{72}$

Figure 4.5 Structures of common UVAs (a) and HALS (b) in clear coats.

Figure 4.6 Structures of (a) HALS and (b) UVA used in reference film.

Figure 4.7 ATR FTIR (left) and Raman (right) spectra of BASF E10CG081G (top) and R10CG392D (bottom) clear coats. Blue, red, green, pink, and purple arrows indicate peaks ascribed to acrylic, urethane, styrene, melamine, and general aromatic structures, respectively.

Figure 4.8 ATR FTIR (left) and Raman (right) spectra of PPG APO (top), NCT (middle), and TMAC (bottom) clear coats. Blue, red, green, pink, and purple arrows indicate peaks ascribed to acrylic, urethane, styrene, melamine, and general aromatic structures, respectively.

Figure 4.9 Subtraction spectra of a BASF R10CG392D sample after 1000 hours of weathering. Lines indicate the peaks used to track oxidation (left) and mass loss (right).

Figure 4.10 MSP spectra of unweathered samples.

Figure 4.11 Structures of the benzotriazole UVAs used in the BASF clear coats (a) E10CG081G and (b) R10CG392D. 
Figure 4.12 Total clearcoat absorbance with UV exposure of black (left) and white (right) samples.

Figure 4.13 Change in YI with dose of BASF (top) and PPG (bottom) samples. Error bars represent the standard variance. Lines are eye guides.

Figure 4.14 Change in $b^{*}$ with dose of BASF (top) and PPG (bottom) samples. Error bars represent the standard variance. Lines are eye guides.

Figure 4.15 DE* between initial and weathered BASF (top) and PPG (bottom) samples. Error bars represent the standard variance. Lines are eye guides.

Figure 4.16 Gloss retention with increasing dose of BASF (top) and PPG (bottom) clear coats. Error bars represent the standard variance. Lines are eye guides. 


\section{Overview}

Paints are a common type of trace evidence in forensic cases as they can be easily transferred in collisions between objects and people. The Laboratory Division of the Federal Bureau of Investigation (FBI) in the U.S. Department of Justice primarily investigates such evidence in the context of automotive paints, noting fragments and smears are often found on clothing in hit-and-run cases. ${ }^{1}$ The Chemistry Unit examines paint for make/model comparisons and/or determinations, and can perform make, model, and year searches for hit-and-run automotive samples. ${ }^{2}$ Analyses typically involve comparison of a sample to a known or control paint chip, which must be collected from the suspected source and have all layers intact down to the substrate. Examinations note the layer structure including the sequence, relative thickness, color, texture, number, and chemical composition of the sample. ${ }^{1} \mathrm{~A}$ hate crime case in 2011 highlighted the capabilities of the unit for conducting an examination on a sample for which a proper control could not be obtained. ${ }^{3}$ Though color and chemical composition could be confirmed with manufacturer contacts and comparisons to database samples, improved availability of laboratory color analysis techniques may have aided in the investigation.

This work seeks to contribute to the development of color measurement and comparison techniques for automotive paints that may be employed by the forensics community. As color can be very subjective, the focus here is to provide a means of quantification by employing coordinates in defined color spaces as descriptors. The microspectrophotometer (MSP) and macroscopic color meter will be examined by obtaining color coordinates of automotive paint fragments using the 
two methods. Since automotive manufacturers primarily employ color meters during production and MSP is used by forensics laboratories to compare known-questioned samples, a study of how the methods correlate would be useful for the development of color databases by manufacturers from which forensic examiners can draw color information for samples lacking controls. This work seeks to develop a standard procedure for quantification of the color of automotive paints using these techniques by defining color with the Commission on Illumination (CIE) $L^{*} a^{*} b^{*}$ color space system.

Spectroscopic techniques are of considerable importance for chemical characterization in forensics examinations, so sample preparation consistency and overall measurement procedure are crucial to ensuring result accuracy. Current protocol involves qualitative comparison of sample to database spectra, so the goal here is to develop a procedure to obtain good quality spectra from which quantitative data can be drawn to aid in specific vehicle identification. FTIR and Raman spectroscopic measurements of automotive paint samples and fragments from standard paint panels will be collected with an emphasis on sample preparation consistency to obtain high quality spectra. Principal component analysis (PCA) may aid in quantification of binder components from spectra and will be employed on the clear coat spectra collected.

Lastly, specific vehicle identification may be improved with information on the physical and chemical degradation measurable in the clear coat of paint samples. The clear coat on vehicles is directly exposed to environmental factors and endures the most damage of the layers in an automotive paint, so quantifying this 
degradation should provide information on sample and vehicle history. Therefore, controlled weathering and characterization of new automotive original equipment manufacturer (OEM) samples containing all layers will be examined for degradation. These results should enhance quantification in forensics examinations of automotive paint samples for color, chemistry, and vehicle history information. 


\section{Spectrophotometric Color Analysis of Automotive Coatings}

\subsection{Introduction}

Forensic scientists are often called upon to identify trace evidence. Paints are a common artifact transferred by objects such as vehicles, clothing, weapons, and people. Automotive coatings are of interest because of their prevalence in common crimes like hit-and-run accidents. Current standards for forensic examinations of paint have been published by ASTM International in the United States, but application of these standards is not universally required by operating forensic science service providers. ${ }^{4}$ In 2013 , this led to the formation of a partnership between the National Commission on Forensic Science in the Department of Justice and the National Institute of Standards and Technology (NIST) to improve the reliability of practices in the forensics community. ${ }^{5}$ The Organization of Scientific Area Committees for Forensic Science at NIST is performing research to assess currently published standards, and developing a registry of approved standards and guidelines which are recommended to be mandatory, but not required. ${ }^{6}$ The present work is intended to determine the utility and associated errors of techniques presented in published standards and those not currently used by the forensics community, but common in the automotive coatings industry.

The Scientific Working Group for Materials Analysis (SWGMAT) has published guidelines summarizing the practices in published standards with the intent of assisting examiners in the forensic analysis of paint. ${ }^{7}$ The goal of forensics examinations is typically either comparison of a questioned sample to a known, potential match, or specific vehicle identification. ${ }^{8}$ An investigation usually begins by 
microscopic examination of the sample for physical properties. For automotive coatings, the sequence of layers present in the specimen is an important characteristic since they typically consist of multiple layers (Figure 2.1). ${ }^{8}$ Each layer has a specific purpose and its presence or thickness may provide information on the year or make of the vehicle. The electrocoat is applied after zinc phosphate treatment by negatively charging the substrate so the positive paint solids deposit as a layer which is then cured and enhances corrosion resistance on metal parts. The primer improves chip resistance of the coating and protects the electrocoat. The primer surfacer can be applied directly on top of the electrocoat, or on a primer for added protection, and is sometimes pigmented to improve depth of color. ${ }^{9}$ The terms primer and primer surfacer will be used here, with primer referring to the layer directly on the substrate. The base coat contains pigments and other additives to give the coating its final appearance; in special effect coatings, this layer may contain metallic flakes or inorganic particles. In some cases, the effect particles are applied as a separate clear layer on top of a flat base coat. The final clear coat protects the coating and substrate from physical and chemical environmental damage, such as ultraviolet (UV) radiation. ${ }^{8}$ In some older pre-1990 paint systems, the clear coat was pigmented for appearance as well as UV protection. ${ }^{10}$ Automotive coatings vary in layer sequence, so microscopic examination can quickly rule out possibilities in known-questioned scenarios. Visual examination is the recommended first course of action by ASTM International, and is currently utilized by forensic laboratories. ${ }^{11}$ However, fragments obtained from crime scenes may not always contain all the layers in the coating. 


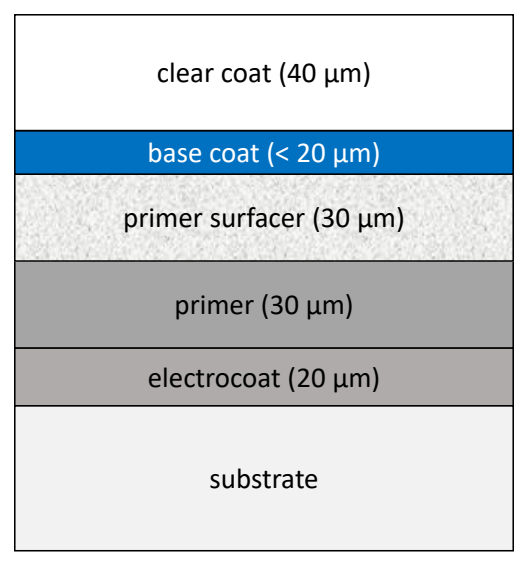

Figure 2.1 Layers comprising a typical automotive coating.

In addition to layer sequence, examiners often note the texture, color, and any other physical characteristics. Color is often an important determinant in known-questioned scenarios. Unfortunately, visual comparisons of samples for color are highly dependent on a variety of factors including the observer, illumination, sample size, viewing distance, and standard. The SWGMAT guidelines recommend use of the International Commission on Illumination (CIE) system of color description, which provides quantitative descriptions for colors in a continuous spatial coordinate system. ${ }^{7}$ The primary benefits of this system include continuity and objectivity, as color is calculated from spectrophotometric data rather than by visual comparison to standards. ${ }^{12}$

The CIE has developed two related coordinate systems that establish color spaces to describe the visual perception of non-self-luminous specimens. ${ }^{13}$ These are the $X, Y, Z$ (tristimulus) and the $L^{*}, a^{*}, b^{*}$ (CIELAB) systems. The CIELAB system was developed in 1976 as an improvement on the tristimulus system because humans perceive differences between darker colors, such as blue and violet, with greater sensitivity than lighter colors, such as green or yellow. ${ }^{13}$ The 
CIELAB color space consists of three axes: $L$ * representing lightness (ranging from black at 0 to white at 100$), a^{*}$ representing green if negative and red if positive, and $b^{*}$ representing blue if negative and yellow if positive; chroma and hue angle can additionally be used to describe the color space (Figure 2.2). ${ }^{13}$

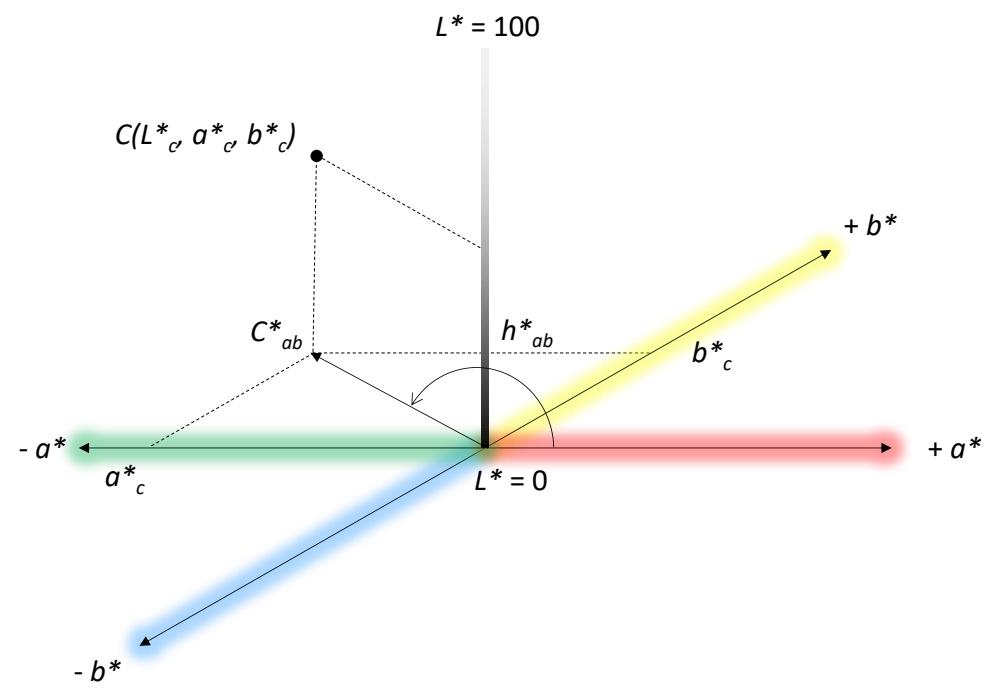

Figure 2.2 Visualization of CIELAB system including chroma and hue angle.

Unfortunately, ASTM standards summarized in published guidelines provide few analysis specifics. ${ }^{11,14,15}$ For instance, the ASTM color standards contain no guidelines for what constitutes two samples as being sufficiently similar to assert the questioned sample originated from the known. ${ }^{16}$ The SWGMAT guide for color determination of paint samples using colorimetry and microspectrophotometry in forensics examinations includes procedural guidelines and refers briefly to the literature citing the colorimetric color difference between different locations of the same vehicle can vary by as much as 1 to 3 CIELAB units. ${ }^{17}$ The simplest CIE color difference formula is shown in Equation 1, and is most commonly used in the current literature to represent the color difference between two automotive coatings. ${ }^{18-20}$ However, visual discrimination of similar colors differs by hue, with non- 
uniformity in the blue region, so $\mathrm{CIE}$ has developed more complex equations to account for these differences and more closely approximate the standard observer's perception of color difference. The most recent of these was established in 2000, and relies on light (L), chroma (C), and hue angle $(\mathrm{H})$ values along with weighting functions (S) and parametric factors $(\mathrm{k})$ for each coordinate to adjust for variation in visual sensitivity under experimental conditions differing from the reference conditions used to develop the equation (Equation 2). ${ }^{21}$ According to CIE, this equation is generally not necessary in industrial practice, so total color difference is typically used as a measurement of observed or perceived difference. Therefore, this equation should not be necessary in forensics laboratories assuming experimental conditions are well controlled. ${ }^{21}$

$$
\begin{aligned}
& \Delta E^{*}=\left[\left(\Delta L^{*}\right)^{2}+\left(\Delta a^{*}\right)^{2}+\left(\Delta b^{*}\right)^{2}\right]^{\frac{1}{2}} \\
& \Delta E_{00}^{*}=\sqrt{\left(\frac{\Delta L^{\prime}}{k_{L} S_{L}}\right)^{2}+\left(\frac{\Delta C^{\prime}}{k_{C} S_{C}}\right)^{2}+\left(\frac{\Delta H^{\prime}}{k_{H} S_{H}}\right)^{2}+R_{T}\left(\frac{\Delta C^{\prime}}{k_{C} S_{C}}\right)\left(\frac{\Delta H^{\prime}}{k_{H} S_{H}}\right)}
\end{aligned}
$$

Since samples in forensic cases are typically fragments or smears, the use of microspectrophotometry (MSP) is heavily emphasized in color examination. Recent literature has shed some light on the use of MSP for color analysis of automotive base coats. However, color determined by MSP often conflicts with color determined by macroscale spectrophotometers used by automotive coating manufacturers with spot sizes on the $5 \mathrm{~cm}^{2}$ scale. ${ }^{17}$ These macroscale spectrophotometers or color meters can be more convenient for examiners in the field as they provide immediate color values and require minimal training and sample prepara- 
tion. Databases of automotive coating spectra have been developed utilizing commercial color meters, so comparison of the two methods is necessary to develop a standard protocol for their use in the field. ${ }^{17,22}$

The foremost complication with forensic color analyses is the presence of metallic flakes or ceramic additives. This is of utmost importance in automotive coating examinations because effect coatings comprise $80 \%$ of the automotive finishes industry. ${ }^{23}$ Metallic flakes reflect light, which can lead to interference in MSP spectra. Their orientation also affects the macroscale appearance of the coating by altering how light is reflected at different viewing angles. ${ }^{23}$ Metallic flakes in a sample also typically increases the lightness coordinate in color meter measurements. Thus, color meters must provide measurements at multiple viewing angles to comprehensively characterize metallic coatings, as described by current ASTM standards. ${ }^{24,25}$ However, these special additives can sometimes be avoided in MSP analysis by utilizing higher magnification objectives and smaller apertures. Similar complications arise with pearlescent coatings. Although color differences obtained by the two methods will never be identical, color meters are low-cost, commercially available, and already widely used by automotive manufacturers. Therefore, the color difference between similar samples calculated from data collected by these two methods should be assessed to determine their utility for initial field use.

Color meters are available in various illumination-observer geometries to account for special effect coatings. According to ASTM standards, color coordinates should be measured with $45^{\circ}$ illumination at three aspecular $\left(15^{\circ}, 45^{\circ}\right.$, and 
$110^{\circ}$ ) detection angles (Figure 2.3)..$^{24}$ Generally, current standards state any objects that change color with observation angle should be described by measurements at five aspecular angles, with $25^{\circ}$ and $75^{\circ}$ recommended in addition to $15^{\circ}$, $45^{\circ}$, and $110^{\circ} .{ }^{25}$ Multi-angle color meters are available and in commercial use; however, the result is color coordinates for each observation angle. Since effect additives in automotive coatings could differ in orientation based on application, sampling location, and other manufacturing variations, this introduces errors in known-questioned comparisons. This method also complicates color difference characterization as each sample would be described by a set of coordinates for each observation angle. However, instruments employing a diffuse illumination geometry utilizing a white coated hemispherical chamber have been designed to evenly illuminate non-uniform, textured samples. In conjunction with $8^{\circ}$ measurement geometry, the effects of stray light, texture and other direction-dependent effects should be minimized. ${ }^{12}$ Therefore, the color meter with spherical diffuse illumination and $8^{\circ}$ measurement geometries with specular component included 
(d/8 spin) will be used for initial comparisons between color meter and MSP color differences.

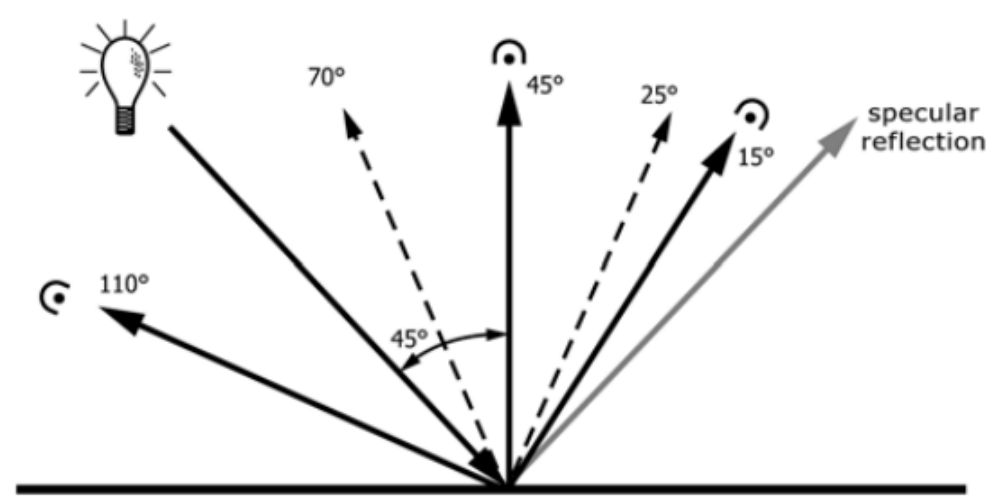

Figure 2.3 Illumination-detection geometries recommended by ASTM E2194 for metal flake pigmented materials. ${ }^{24}$

Recent literature has demonstrated the use of MSP for color analysis of automotive base coats. MSP spectra can be collected in transmission (T) mode, or reflectance $(R)$ mode on the cross section (RCS) or surface (RS) of a specimen. T-MSP is typically performed on cross sections prepared by microtomy; this can be significantly more time-consuming and require additional training. ${ }^{18,19}$ However, studies employing all three techniques on the same samples have found transmission spectra are more varied than reflectance spectra, and may permit differentiation between extremely similar, but different samples more effectively than only reflectance mode. The authors of these studies recommend use of transmission in conjunction with at least one reflectance technique. ${ }^{18}$ RS-MSP directly measures samples in-situ after cleaning the surface, which is the most comparable of all three methods to color meters in terms of working principle. MSP spectra of automotive base coats have been collected for special effect coatings of various hues and 
neutrals (white, grey, and black). ${ }^{26}$ Analysis of neutral coatings is especially important since the popularity of automotive coating colors reported at the International Forensic Science Managers Symposium in 2013 in descending order were white, black, silver, and grey before hues like red and blue. ${ }^{27}$ Though spectra of grey and black paint with notable features have been obtained in transmission mode, white base coats yield only noise in the visible region; this was explained by the scattering ability of brightening pigments typically used in white paints such as titanium dioxide. ${ }^{26} \mathrm{~A}$ group at the Institute of Forensic Research in Krakow have obtained CIELAB coordinates from their spectra of non-neutral automotive base coats, and used the color difference formula to gauge the sensitivity of the method for discriminating similar samples and replicates of the same sample. ${ }^{18,19,28}$ This group found most significant differences in transmission spectra between similar colored paints. Forensic examinations should require R-MSP measurements to compare with color meter measurements, as well as T-MSP measurements for analysis of significant spectral differences. The goals here were to (1) quantify the errors associated with color differences calculated from color meter, RS-MSP, and T-MSP measurements, and (2) provide recommendations for utilizing these instruments in forensic laboratories.

\subsection{Experimental}

Automotive coating samples were chosen to represent a variety of coating types: different hues, substrates, and flat and effect (Table 2.1). Samples ending with a "P" were unexposed standards obtained as full systems (clear coat, base 
coat, primer surfacer, primer) on steel ACT panels, numbered with their corresponding sample. The remaining samples were obtained as fragments or as automobiles parts with unknown histories from which fragments were removed. Each sample is categorized according to three general layer sequences (Figure 2.4). Refinish indicates the number of duplicate base coat/clear coat layers on top of the first clear coat closest to the substrate.

A

\begin{tabular}{|c|}
\hline CLEAR COAT \\
\hline BASE COAT \\
\hline PRIMER SURFACER \\
\hline PRIMER (ELECTROCOAT) \\
\hline
\end{tabular}

B

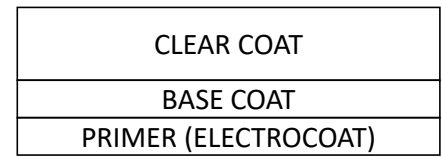

C

\begin{tabular}{|c|}
\hline CLEAR COAT \\
\hline EFFECT (METALLIC FLAKE) LAYER \\
\hline PRIMER SURFACER \\
\hline PRIMER (ELECTROCOAT) \\
\hline
\end{tabular}

Figure 2.4 General layer sequences observed in the sample set.

Table 2.1 Automotive Coatings Examined

\begin{tabular}{|c|c|c|c|c|c|c|c|c|}
\hline Sample & Make & Country & Year & Substrate & Color & Effect & Refinish & Sequence \\
\hline $1 \mathrm{~A}$ & VW & Germany & 2012 & metal & white & metallic & 1 & C \\
\hline $2 A^{*}$ & VW & Germany & 2012 & plastic & white & metallic & 2 & C \\
\hline 3 & Toyota & Japan & 2005 & metal & red & metallic & 0 & A \\
\hline 10 & Dodge & US & 2007 & metal & black & metallic & 0 & $B$ \\
\hline $10 \mathrm{P}^{*}$ & N/A & $\mathrm{N} / \mathrm{A}$ & $\mathrm{N} / \mathrm{A}$ & metal & black & flat & $\mathrm{N} / \mathrm{A}$ & $A$ \\
\hline $11^{*}$ & Dodge & US & 2007 & plastic & black & metallic & 1 & B \\
\hline $12^{*}$ & Saab & Sweden & 2001 & metal & red & flat & 1 & $A$ \\
\hline $13^{*}$ & Saab & Sweden & 2001 & plastic & red & flat & 2 & $B$ \\
\hline $15^{*}$ & Scion & Japan & 2006 & metal & black & metallic & 0 & A \\
\hline $17^{*}$ & Scion & Japan & 2006 & plastic & black & metallic & 0 & $\mathrm{~B}$ \\
\hline 18 & Ford & US & 2006 & metal & red & metallic & 0 & $\bar{A}$ \\
\hline $18 A^{*}$ & Ford & US & 2013 & metal & blue & metallic & 1 & $A$ \\
\hline $19 A^{*}$ & Ford & US & 2013 & plastic & blue & metallic & 0 & $B$ \\
\hline 34PS* & $\mathrm{N} / \mathrm{A}$ & $\mathrm{N} / \mathrm{A}$ & $\mathrm{N} / \mathrm{A}$ & metal & blue & metallic & $\mathrm{N} / \mathrm{A}$ & $A$ \\
\hline 34PW* & $N / A$ & $\mathrm{~N} / \mathrm{A}$ & $\mathrm{N} / \mathrm{A}$ & metal & blue & metallic & $N / A$ & $A$ \\
\hline $34^{*}$ & Ford & US & 2014 & metal & blue & metallic & 1 & $A$ \\
\hline $35^{*}$ & Ford & US & 2014 & plastic & blue & metallic & 0 & $A$ \\
\hline 58 & Dodge & US & 2013 & metal & white & metallic & 0 & $A$ \\
\hline $58 \mathrm{P}^{*}$ & $\mathrm{~N} / \mathrm{A}$ & $\mathrm{N} / \mathrm{A}$ & $\mathrm{N} / \mathrm{A}$ & metal & white & flat & $N / A$ & $\mathrm{~A}$ \\
\hline $59^{*}$ & Dodge & US & 2013 & plastic & white & metallic & 1 & $B$ \\
\hline D782 & Mazda & Japan & 2014 & plastic & grey & metallic & 0 & $B$ \\
\hline D786 & Chevrolet & US & 2012 & metal & grey & metallic & 0 & $A$ \\
\hline
\end{tabular}


CIELAB coordinates of the samples were obtained with a BYK spectroguide sphere gloss color meter employing $\mathrm{d} / 8$ geometry with the specular component included, using D65 illuminant and $10^{\circ}$ standard observer functions. The instrument aperture was $11 \mathrm{~mm}$ in diameter, so only samples at least this large and the standard panels were measured; these samples are indicated with asterisks in Table 2.1. Samples were gently cleaned with distilled water and lint-free wipes prior to measurement. Ten measurements at randomly chosen locations were taken per sample. The instrument collected spectral data from $400 \mathrm{~nm}$ to $700 \mathrm{~nm}$ in $10 \mathrm{~nm}$ increments to calculate color coordinates according to ASTM E308..$^{29,30}$

For microscopic analysis, spectra were obtained from $380 \mathrm{~nm}$ to $780 \mathrm{~nm}$ (0.4 nm step size) on a CRAIC MP-2 microspectrophotometer. Wavelength and photometric accuracies were confirmed with NIST traceable holmium oxide, didymium oxide, and neutral density filters at the start of each session. The $36 \mathrm{X}$ objective (NA 0.5) was preferred and a variable aperture employed to avoid metallic flakes in effect samples when measuring cross sections. For each mode of MSP analysis, ten measurements were taken at random locations. Dark and reference scans were collected prior to each sample. Spectra were obtained in percent transmittance, and CRAIC ColorPro software used to obtain CIELAB coordinates from each spectrum. These were calculated using the $400 \mathrm{~nm}$ to $700 \mathrm{~nm}$ spectral data with D65 illuminant and $10^{\circ}$ standard observer functions.

For reflectance-mode MSP (R-MSP), measurements were made on the surface of the paint chip in situ. Samples were cleaned with methanol and lint-free wipes, and taped to a glass slide for analysis. For transmission-mode MSP 
(T-MSP), a fragment of each sample was embedded in epoxy (LECO resin and hardener, 5 to 1 by mass), cured at $40^{\circ} \mathrm{C}$ overnight, and microtomed at room temperature to obtain cross sections of $5 \mu \mathrm{m}$ thickness. The cross sections were transferred to a quartz slide, flattened with an Excel roller, and mounted with anhydrous glycerol and a quartz coverslip. The coverslip was then flattened with an Excel roller immediately prior to analysis to remove any air pockets.

To calculate the color difference between sample pairs, the CIELAB coordinates for the ten measurements on each sample were split into pairs to form five groups, and each pair averaged. This was due to the lack of true replicates available for each sample as most were obtained from actual vehicles. The five average coordinates were used to calculate five $\mathrm{DE}^{*}$ values for each sample pair. The average and standard deviation of the $\mathrm{DE}^{*}$ values was used to compare the uncertainty in color differences obtained by the color meter, R-MSP, and T-MSP methods. JMP software was used to perform correlation analyses on the $\mathrm{DE}^{\star}$ obtained by the three methods to compare the methods.

\subsection{Results and Discussion}

The average color differences, $\mathrm{DE}^{\star}$, and standard deviations are listed in Table A.1 and Table A.2 of the Appendix, respectively. In both tables, values for each pair from top to bottom correspond to the color meter, R-MSP, and T-MSP measurements. For T-MSP, the CIELAB coordinates for the base coat closest to the surface were used in calculations. The samples are grouped by color (white, black, blue, and red). Sample pairs obtained from the same vehicle at different locations are boxed in black, and sample/matching standard pairs are boxed in red. The 
objective was to gauge similarities between R-MSP and color meter measurements as they both detect reflected light, and between CM and T-MSP as T-MSP spectra have been shown to be more variable.

For an individual vehicle, samples from different locations should ideally have $\mathrm{DE}^{\star}$ close to zero, indicating no color difference within a vehicle. However, this is unlikely due to variations in manufacture. Four sample pairs from the same vehicle were sufficiently large for $\mathrm{CM}$ measurements (Table 2.2). Using $\mathrm{DE}^{\star}$ less than one as the criteria for two samples being from the same source, only one metallic blue pair and the metallic black pair were from the same vehicle based on CM measurements. The general increase in $\mathrm{DE}^{*}$ between $\mathrm{CM}$ and MSP measurements for these samples may be explained by pigment dispersion and other heterogeneities magnified on the microscale in MSP. However, the flexible paint applied to peripheral regions of automobiles is also commonly manufactured by different suppliers than the body, so some color difference may be expected. ${ }^{31}$ Overall, $\mathrm{DE}^{\star}$ determined by R-MSP was lower than T-MSP, except for the flat red pair. This is likely because focus and sample location was controlled in R-MSP to avoid scratched or rough areas of the samples, which influenced the CM measurements. Finally, TMSP DE* only exceeded 10 for the metallic black pair, likely due to lack of significant features in MSP spectra of neutral coatings attenuating the noise in these spectra. Qualitative examination of T-MSP spectra can provide evidence neutral or heavily damaged samples are from the same vehicle when $\mathrm{CM} \mathrm{DE}^{*}$ is greater than R-MSP DE* and T-MSP DE* is unusually large. 
Table 2.2 DE* of Sample Pairs from Same Vehicle

\begin{tabular}{cc|ccc} 
& & \multicolumn{3}{|c}{ Mean \pm Std. Dev. } \\
\hline Color & Pair & CM & R-MSP & T-MSP \\
\hline Red & $12 / 13$ & $5.14 \pm 0.43$ & $2.10 \pm 0.25$ & $9.37 \pm 1.51$ \\
Blue & $18 \mathrm{~A} / 19 \mathrm{~A}$ & $0.42 \pm 0.09$ & $5.32 \pm 3.73$ & $5.76 \pm 1.20$ \\
Blue & $34 / 35$ & $1.49 \pm 0.08$ & $4.26 \pm 1.94$ & $7.91 \pm 1.59$ \\
Black & $15 / 17$ & $0.46 \pm 0.08$ & $7.51 \pm 1.23$ & $34.33 \pm 1.89$
\end{tabular}

The shapes of the T-MSP spectra of pairs 18A / 19A and 12 / 13, respectively, are very similar (Figure 2.5). The minor variations in intensity can be attributed to heterogeneities in pigment dispersion at the ten measurement locations, and sample preparation variations. Though T-MSP DE ${ }^{\star}$ for pair 12 / 13 was 9 , the shape of the actual spectra are quite similar. The discrepancy between the calculated $D^{*}$ value and qualitative similarity of the spectra illustrate why qualitative inspection of TMSP spectra is critical in pairwise comparisons in addition to comparison of CM and R-MSP DE* values. The combined methods of color meter and MSP in both modes provide a more complete characterization of the color differences between two samples than any one measurement alone. 

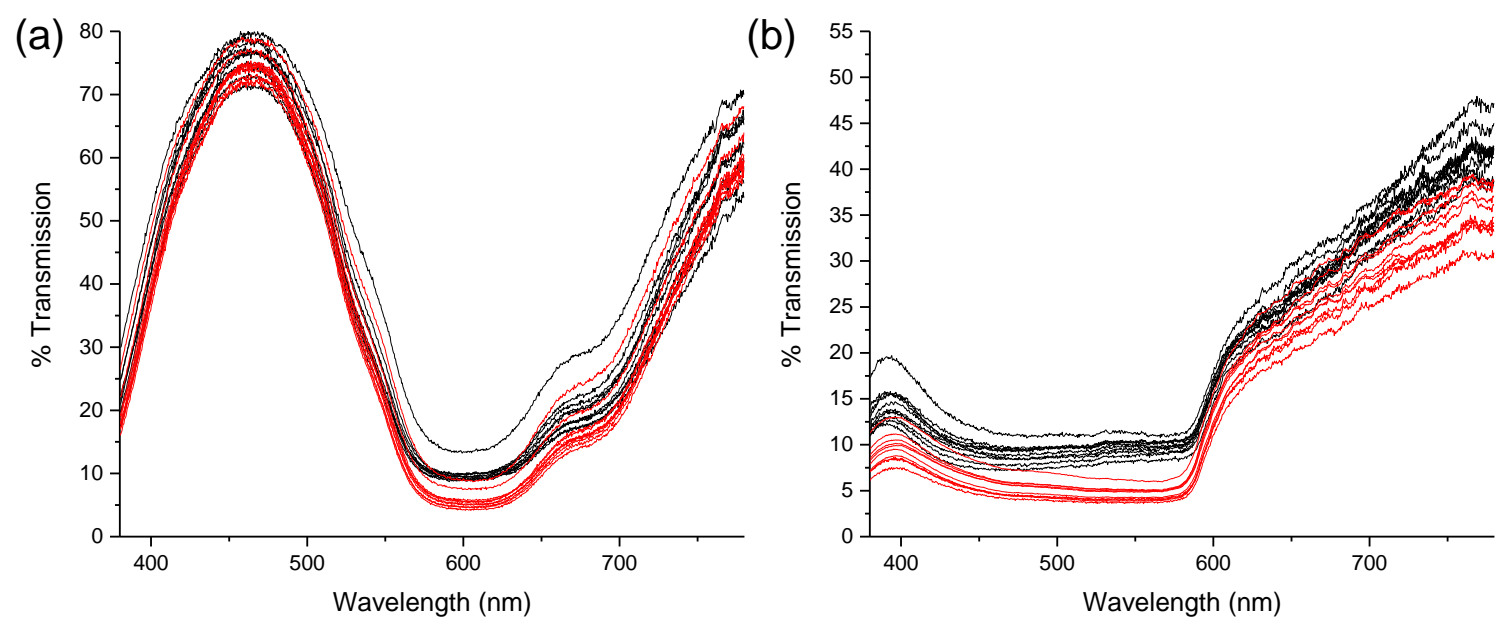

Figure 2.5 T-MSP spectra of (a) metallic blue samples 18A (black) and 19A (red), and (b) flat red samples 12 (black) and 13 (red).

Within each color group, $\mathrm{CM} \mathrm{DE}^{\star}$ between red, blue, and black samples was less than 10 , and R-MSP DE* less than 12 . As the purpose of this investigation was to gauge the utility of the handheld color meter in initial field color comparisons, the similarity in $\mathrm{DE}^{*}$ between the color meter and R-MSP measurements for these hues is promising. The examined metallic blue samples were all intended to be the same color, either from different parts of the same vehicle, different vehicles of the same color, or standard panels of the color (Table 2.3). DE* was less than 10 in most cases, and less than 5 for $\mathrm{CM} \mathrm{DE}^{*}$. The comparatively larger $\mathrm{CM} \mathrm{DE}^{*}$ for sample pairs including 34 are likely because this sample was barely large enough for $\mathrm{CM}$ measurements (11-mm diameter circular aperture), so edge irregularities contributed error. 
Table 2.3 DE* of Same Color Metallic Blue Samples Examined

Mean \pm Std. Dev.

\begin{tabular}{ccccl}
\hline Pair & CM & R-MSP & T-MSP & \multicolumn{1}{c}{ Relation } \\
\hline 18A / 19A & $0.42 \pm 0.09$ & $5.32 \pm 3.73$ & $5.76 \pm 1.20$ & same vehicle \\
34 / 35 & $1.49 \pm 0.08$ & $4.26 \pm 1.94$ & $7.91 \pm 1.59$ & same vehicle \\
18A / 34 & $1.21 \pm 0.08$ & $7.78 \pm 4.14$ & $6.41 \pm 2.90$ & same color, different vehicle \\
19A / 35 & $1.78 \pm 0.24$ & $4.26 \pm 2.35$ & $5.84 \pm 1.49$ & same color, different vehicle \\
34 / 34PS & $2.39 \pm 0.10$ & $6.11 \pm 1.15$ & $6.76 \pm 1.11$ & standard panel for color \\
34 / 34PW & $3.08 \pm 0.14$ & $7.46 \pm 3.55$ & $6.74 \pm 2.10$ & standard panel for color \\
35 / 34PS & $0.97 \pm 0.16$ & $7.76 \pm 1.14$ & $9.99 \pm 1.51$ & standard panel for color \\
35 / 34PW & $1.77 \pm 0.11$ & $8.70 \pm 4.01$ & $11.77 \pm 0.72$ & standard panel for color \\
34PS / 34PW & $0.89 \pm 0.15$ & $5.12 \pm 2.56$ & $4.19 \pm 1.06$ & standard panels for same color
\end{tabular}

An important consideration in MSP analysis of neutral colors is the lack of large, characteristic peaks in the spectra. For example, the metallic black and white pairs from the same vehicle lack any major peaks (Figure 2.6 a). The T-MSP DE* of black pair 15 / 17 was significantly greater than 10 (34) because of this lack of major peaks combined with the difference in intensity. It is possible these two samples were formulated separately as they are from different areas of the same car, but this does not warrant $\mathrm{DE}^{*}$ of this magnitude. The $\mathrm{CM} \mathrm{DE}^{\star}(<1)$ and R-MSP $\mathrm{DE}^{\star}(<10)$ measurements were more reliable in this case. Thus, for non-white 
neutral samples, color meter and R-MSP measurements should be used primarily to gauge color difference.
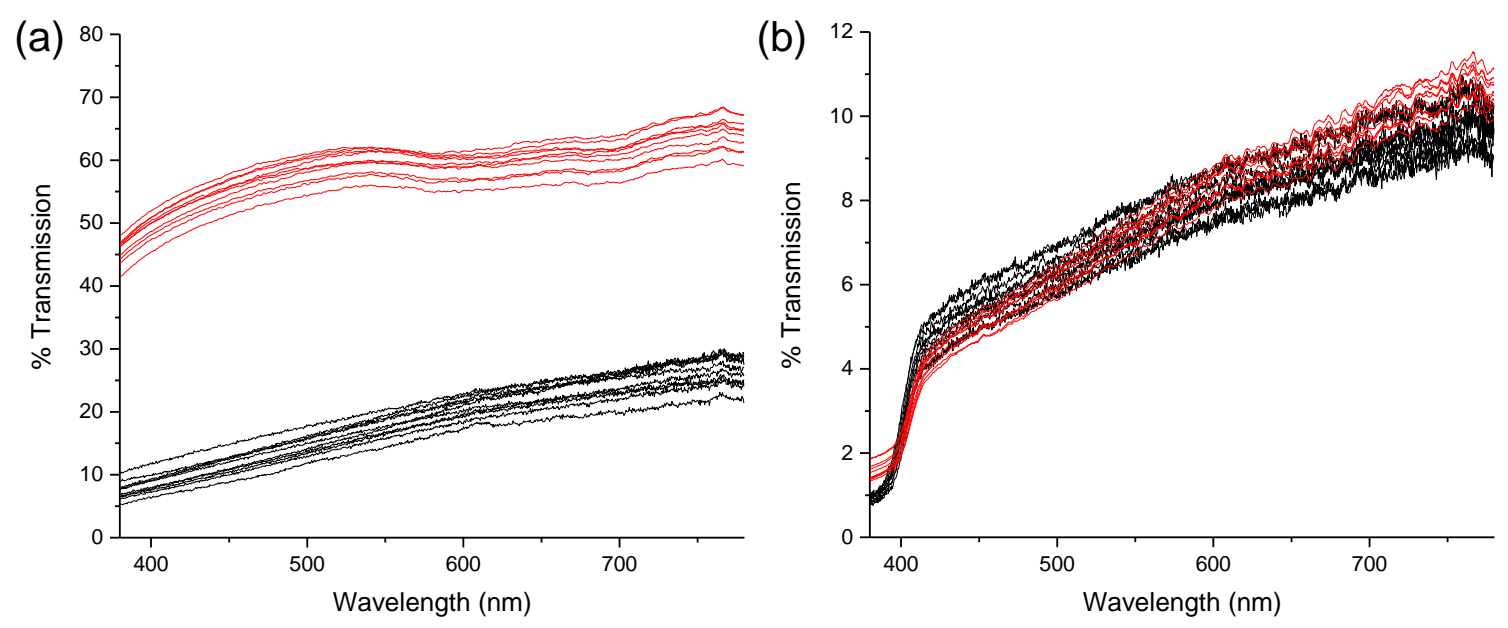

Figure 2.6 T-MSP spectra of (a) metallic black samples 15 (black) and 17 (red), and (b) metallic white samples 58 (black) and 59 (red).

The white samples exhibited similar T-MSP spectra with no distinguishing peaks (Figure 2.6 b). White base coats also contain light-scattering pigments, which decrease transmission and signal-to-noise. This resulted in similar T-MSP $\mathrm{DE}^{*}$ for the different color pair, 2A / 58P, (9) and the same color pair, 59 / 58P (4). In the literature, similar issues with inadequate variation in T-MSP spectra of white samples have been discussed. ${ }^{26}$ R-MSP $D^{*}$ for the white samples was also slightly larger than R-MSP DE* for the other colors. This was likely because the two samples, $2 \mathrm{~A}$ and 59 , were highly metallic (Figure 2.7). The effect of metallic flakes is averaged by the large aperture of the color meter, but impossible to avoid in R-MSP; as a result, R-MSP DE* was 17 for pair 58P / 59, but CM DE* was less 
than 5. Therefore, R-MSP analysis of highly metallic samples and T-MSP analysis of white samples is not recommended.

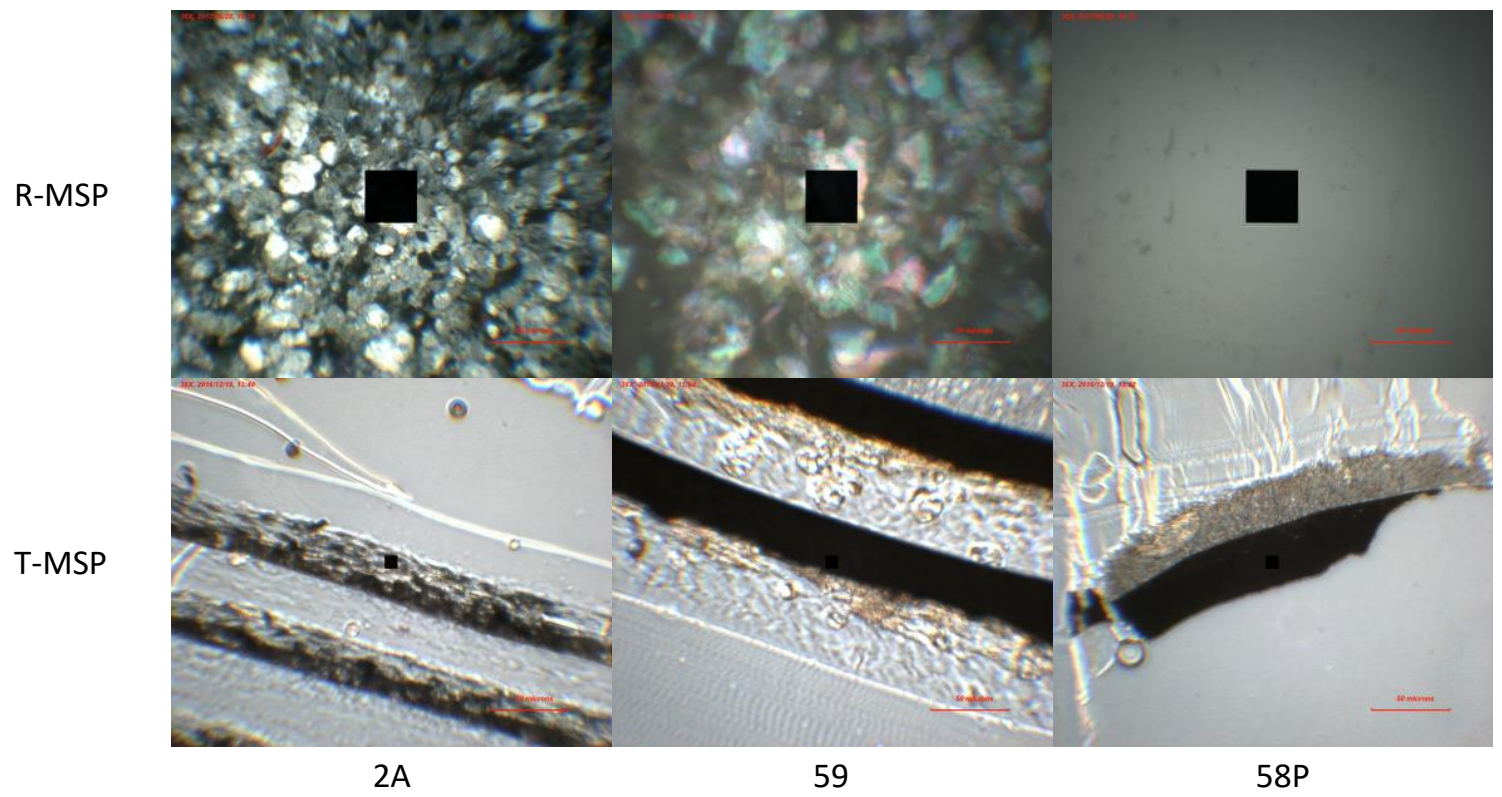

Figure 2.7 White samples measured by MSP in reflection and transmission modes.

$\mathrm{CM}$ and $\mathrm{T}-\mathrm{MSP} \mathrm{DE}^{*}$ of the black and white samples with corresponding standard panels can be examined to gauge the influence of metallic flakes on color as the standard panels were not produced with special effect additives (Table 2.4). The color meter measurements were not significantly impacted by the presence of metallic flakes, as $\mathrm{CM} \mathrm{DE}^{*}$ was less than 5 in both cases, comparable to $\mathrm{CM} \mathrm{DE}^{*}$ for flat samples 12 and 13 from the same vehicle. Although T-MSP DE* for the black pair was large (28), this $\mathrm{DE}^{*}$ value is comparable to T-MSP DE* for the black metallic pair 15 / 17 from the same vehicle. Likewise, T-MSP DE* for the white pair was under 5, comparable to the T-MSP DE* for the white metallic pair 58 / 59 from the same vehicle. The absence of metallic flakes does not appear to influence TMSP DE*; this may be due to the diffuse illumination principle of the color meter 
used. ${ }^{32}$ Therefore, T-MSP may be useful for comparing metallic questioned samples to standard panels from manufacturers lacking special effect additives.

Table 2.4 DE* of Flat / Metallic Standard / Sample Pairs

\begin{tabular}{cc|ccc} 
& & \multicolumn{3}{|c}{ Mean \pm Std. Dev. } \\
\hline Color & Pair & CM & R-MSP & T-MSP \\
\hline black & $10 \mathrm{P} / 11$ & $1.71 \pm 0.08$ & $1.13 \pm 0.67$ & $28.32 \pm 1.05$ \\
white & $58 \mathrm{P} / 59$ & $4.21 \pm 0.18$ & $19.18 \pm 2.39$ & $4.46 \pm 1.18$
\end{tabular}

Error in $\mathrm{DE}^{\star}$ as quantified by standard deviation did not exceed 0.4 for the color meter measurements, and was less than 5 for the MSP measurements. The highest standard deviations were observed for R-MSP DE* of highly metallic blue samples. Heterogeneities in pigment and metal flake dispersion were the cause of this variation; these could not be avoided even by varying aperture and carefully choosing sample location. However, these additives were primarily aligned parallel to the sample surface, and could be avoided in T-MSP. Despite greater variation in T-MSP DE* compared to CM DE* standard deviation was roughly 3 or less in most cases except the metallic neutral samples 17 and 2A. Qualitatively, T-MSP displays good repeatability in spectral shape as previously discussed.

Multivariate nonparametric correlation analysis was performed on $\mathrm{DE}^{*}$ to compare the color meter to the MSP because $\mathrm{DE}^{\star}$ for this sample set was not normally distributed (Figure 2.8). Spearman's $\rho$ ranges from -1 to 1 and provides a measurement of correlation between variables (Table 2.5). $\rho$ is close to unity (0.88) for the CM and R-MSP DE* comparison, providing evidence for a correlation. The evidence for correlation between $\mathrm{DE}^{*}$ determined by T-MSP and the two reflectance methods is not as strong. This difference in correlation may be because 
CM and R-MSP both operate by reflectance while T-MSP operates via transmission. Other factors such as differences in instrumentation, and non-uniformity of $D E^{\star}$ for hues of different lightness (e.g. blue versus yellow) likely contribute to the differences in correlation between the methods.

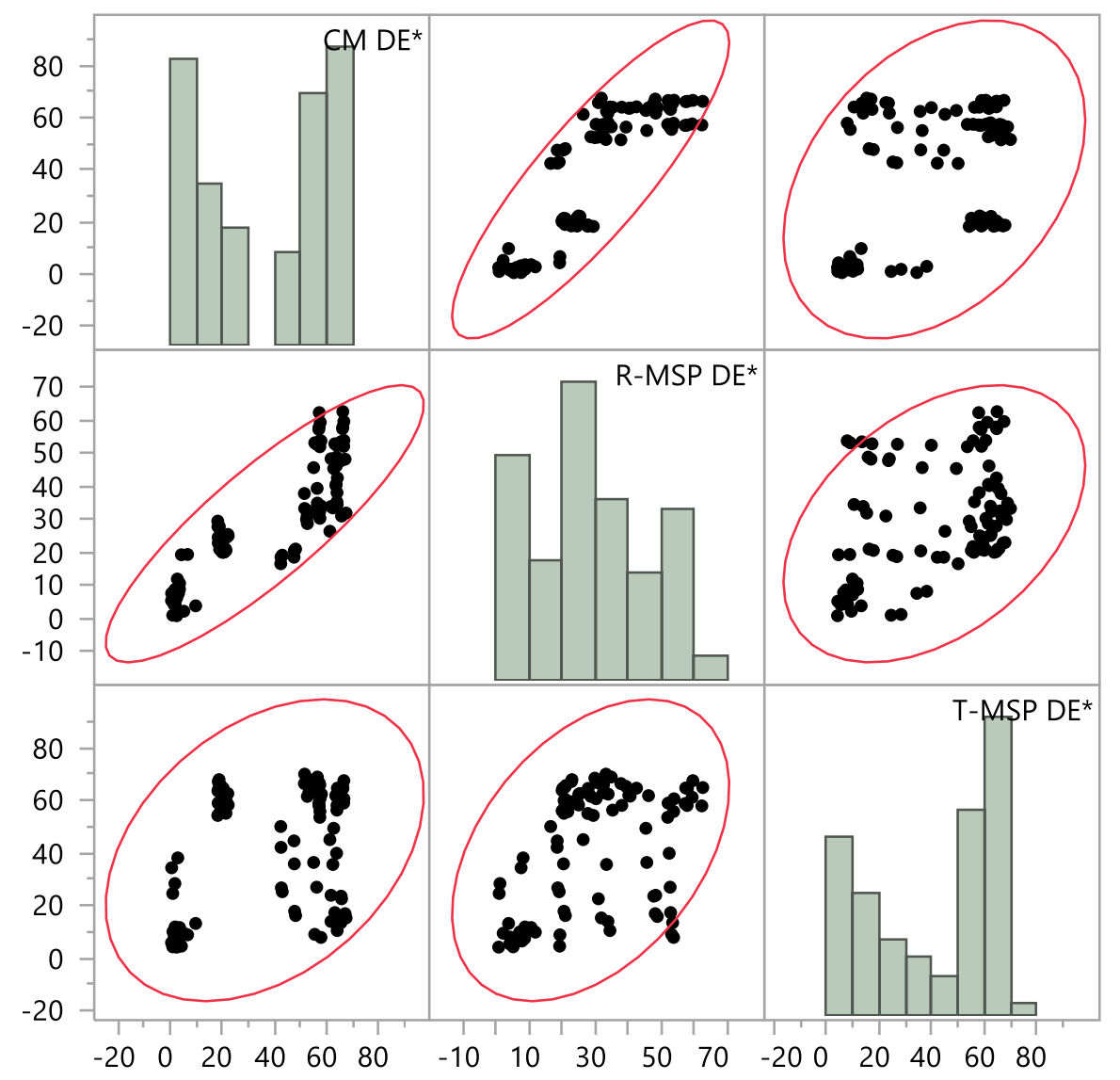

Figure 2.8 Scatterplot matrix and histograms of CM, R-MSP, and T-MSP DE*, indicating lack of normality.

Table 2.5 Correlation Coefficients for Color Meter and MSP DE*

\begin{tabular}{c|c|c|c} 
Method 1 & Method 2 & Spearman $\rho$ & p-value \\
\hline R-MSP & CM & 0.88 & $<0.0001$ \\
\hline T-MSP & CM & 0.38 & $<0.0001$ \\
\hline T-MSP & R-MSP & 0.47 & $<0.0001$
\end{tabular}




\subsection{Conclusions}

$D E^{*}$ is frequently used to quantify color difference of automotive coatings and may be determined from spectrophotometric data gathered by a color meter as in the industry or microspectrophotometer in forensics. For samples from the same vehicle, $C M D^{*}$ and $\mathrm{R}-\mathrm{MSP} \mathrm{DE}^{*}$ were less than 1 and 5 , respectively, and T-MSP spectra were qualitatively similar. This indicates samples obtained from the same vehicle analyzed by R-MSP should yield low $D^{\star}$ (quantitatively similar) via this method. For metallic samples and their flat, corresponding standards, T-MSP DE* was similar to T-MSP $\mathrm{DE}^{\star}$ for same color metallic sample pairs from the same vehicle. Therefore, T-MSP is a good technique for avoiding the influence of effect additives. Though the two microspectrophotometric methods utilize the same instrumentation, the data showed a stronger correlation between $\mathrm{CM} \mathrm{DE}^{*}$ and $\mathrm{R}$ MSP than between CM DE* and T-MSP. Though a larger, more diverse sample set is required to quantify this correlation for practical use, this data illustrated color

meter analysis may be useful in initial field analyses of automotive coating fragments for forensic purposes. 


\section{Spectroscopic Analysis of Automotive Coatings}

\subsection{Introduction}

After examination of physical characteristics, chemical analysis is necessary to further assess the likelihood that a questioned sample matches a known. Spectroscopic analysis via Fourier transform infrared (FTIR) spectroscopy, Raman spectroscopy, x-ray fluorescence (XRF) spectroscopy, and scanning electron microscopy-energy dispersive x-ray (SEM-EDX) spectroscopy have historically been utilized. ${ }^{11,33,34}$ The Federal Bureau of Investigation (FBI) and ASTM forensics standards provide guidelines for other analytical methods such as pyrolysis gas chromatography-mass spectrometry (PGC-MS), and fluorescence microscopy. ${ }^{11}$ However, the overwhelmingly most utilized method of chemical analysis for forensic examinations of automotive coatings remains FTIR spectroscopy. This has encouraged the development of spectral databases for the resins, pigments, and other additives present in these samples. Recent literature has focused on employing two or more spectroscopic methods in conjunction to differentiate pigments that provide similar spectra by a single method. Raman spectroscopy complements FTIR spectroscopy by detecting the polarizability change in symmetric molecules as the latter can detect only changes in dipole moment in asymmetric molecules. FTIR spectroscopy is typically used to identify binder chemistry and major fillers, while Raman spectroscopy is used to characterize pigments present.

Compounds that impart color to the base coat of automotive coatings can be inorganic or organic pigments, or organic dyes. Specific definitions of "dye" and "pigment" are provided by the Ecological and Toxicological Association of Dyes 
and Organic Pigment Manufacturers (ETAD) and Color Pigment Manufacturers Association (CPMA), but the term pigment is frequently used in the automotive coatings literature to describe all color-imparting additives. ${ }^{35}$ In neutral coatings, inorganic fillers are often used in addition to pigments to increase the solids content and hiding power as they are typically less expensive. Common inorganic fillers employed that have been analyzed by FTIR spectroscopy are clays such as talc and kaolin, barium sulfate, calcium carbonate, anatase and rutile titanium dioxide, and natural and synthetic silica. ${ }^{33,36,37}$ Inorganic pigments used in blue and green base coats such as iron ferrocyanide have also been discussed in the literature. ${ }^{38}$ Yellow, orange, and red inorganic pigments such as nickel titanate, chrome titanate, and hydrous ferric oxide have been studied. ${ }^{34}$ However, organic pigments are sometimes used in conjunction with inorganics in the same base coat to achieve certain colors or avoid health hazards associated with common pigments. For example, chrome yellow was replaced by isoindoline yellow with hydrous ferric oxide and bismuth vanadate in the 1990s because of health concerns. ${ }^{34}$

Spectra become complex with multiple pigments, and organic pigments tend to exhibit lower intensity peaks in FTIR spectroscopy. This can make identifying organic pigments in spectra of unknowns difficult due to the dominance of resin and inorganic pigment peaks. ${ }^{36}$ Furthermore, some inorganic pigments are symmetrical coordination complexes such as phthalocyanine blue, which do not produce signals in FTIR spectra. ${ }^{39}$ Thus, FTIR spectroscopy is not sufficient for characterizing all automotive coating samples. Therefore, Raman spectroscopy is an important complementary technique for base coat pigment analysis. 
Raman spectroscopy has many additional advantages over FTIR spectroscopy. Generally, spectra can extend to lower frequencies well below $600 \mathrm{~cm}^{-1} \mathrm{com}$ pared to the roughly $600 \mathrm{~cm}^{-1}$ lower limit of mercury cadmium telluride (MCT) detectors used in FTIR spectrometers. ${ }^{39}$ This is important because many pigments exhibit distinct bands in the lower frequency region around $500 \mathrm{~cm}^{-1}$ to $600 \mathrm{~cm}^{-1}$. Furthermore, Raman bands generally do not overlap and spectra are not affected by environmental interferences such as carbon dioxide and water. ${ }^{39}$ Current literature illustrates the discriminating power of FTIR and Raman spectroscopies combined for characterizing the binder and pigments of automotive base coats. ${ }^{34,40-45}$ In one study, the low intensity peaks from an organic pigment in the IR spectrum of a red automotive base coat were clearly identified in the Raman spectrum of the sample. ${ }^{36}$

Nonetheless, FTIR spectroscopy is the primary method of binder identification in the literature. As the primary purpose of base coats is aesthetic, the binder chemistries used can vary greatly. In one study of 52 automotive coatings, eight binder chemistries were identified: amino, acrylic, epoxy, vinyl perchloride, nitrocellulose, alkyd, and polyurethane. ${ }^{40}$ Binder chemistries can be identified based on prior analysis of different binder types due to subtle differences in the fingerprint region below roughly $1800 \mathrm{~cm}^{-1} .{ }^{10}$ Binders of coatings result in fewer peaks of higher intensity compared to pigments due to their relative abundance. Therefore, characteristic peaks can be ruled out to permit identification of inorganic pigment peaks. ${ }^{40}$ Manufacturers also have tendencies to preferentially utilize certain binder chemistries, and these have been noted in the literature ( 
Table 3.1). ${ }^{10}$ These chemistries differ slightly when coatings are refinished; nitrocellulose lacquers (sometimes acrylic-modified), solution acrylic lacquers, alkyd, acrylic-alkyd, acrylic-urethane, and flexible urethane enamels are common. Urethane hardeners are also often used with the refinish acrylic and alkyd enamels employed.

Table 3.1 Original Finish Binders on Current Vehicles ${ }^{10}$

\begin{tabular}{|c|c|c|c|}
\hline \multirow{3}{*}{ Acrvlic lacauers } & \multirow[t]{2}{*}{ Domestic } & \multicolumn{2}{|c|}{ Foreign } \\
\hline & & Nonmetallic & Metallic \\
\hline & \multicolumn{3}{|c|}{ Acrylic lacquers } \\
\hline solution & GM cars & & \\
\hline Acrylic-melamine enamels & & $\begin{array}{l}\text { Some Asian man- } \\
\text { ufacturers (Nis- } \\
\text { san, Honda, Hyun- } \\
\text { dai, Subaru) }\end{array}$ & $\begin{array}{l}\text { Most Asian base } \\
\text { and clear coats } \\
\text { (Honda, Mazda, Nis- } \\
\text { san, Mitsubishi, } \\
\text { Subaru, Suzuki, } \\
\text { Toyota) } \\
\text { Most European clear } \\
\text { coats } \\
\text { Some European } \\
\text { base coats }\end{array}$ \\
\hline $\begin{array}{l}\text { solution } \\
\text { dispersion } \\
\text { waterborne }\end{array}$ & $\begin{array}{l}\text { All cars and trucks } \\
\text { All cars and trucks } \\
\text { GM in California }\end{array}$ & & \\
\hline Polyester-melamine enamels & Ford & $\begin{array}{l}\text { Some Asian non- } \\
\text { clear coat models } \\
\text { (Honda, Nissan, } \\
\text { Toyota) }\end{array}$ & $\begin{array}{l}\text { Most European base } \\
\text { coats } \\
\text { Some European } \\
\text { clear coats (BMW, } \\
\text { Fiat, SAAB, VW, } \\
\text { Volvo) }\end{array}$ \\
\hline Polyurethane enamels & All flexible fascia & & \\
\hline \multirow{2}{*}{ Alkyd-melamine enamels } & & $\begin{array}{l}\text { Many Asian manu- } \\
\text { facturers (Honda, } \\
\text { Mazda, Nissan, } \\
\text { Mitsubishi, Sub- } \\
\text { aru, Toyota) }\end{array}$ & $\begin{array}{l}\text { Some Mercedes- } \\
\text { Benz with acrylic- } \\
\text { melamine clear } \\
\text { coats }\end{array}$ \\
\hline & & $\begin{array}{l}\text { Most European } \\
\text { manufacturers } \\
\text { (BMW, Fiat, Mer- } \\
\text { cedes-Benz, MG, } \\
\text { SAAB, VW, Volvo) }\end{array}$ & \\
\hline
\end{tabular}

Clear coats analysis is often for subtle differences in the binder chemistry, such as the presence or quantity of hardener or crosslinking component. Sample 
preparation is simpler compared to full system analysis due to the absence of lightabsorbing pigments. Sample preparation methods reported in the literature utilize scalpels to obtain scrapings of the clear coat off samples; however, this method requires a large sample to obtain sufficient scrapings. ${ }^{46-48}$ ATR FTIR spectroscopy can also be performed on the sample in situ as the depth of penetration of common crystals is sufficiently shallow compared to the thickness of most clear coats.

Currently, high resolution FTIR spectra of common automotive pigments are available on the SWGMAT website, and the Federation of Societies for Coatings Technology published An Infrared Spectroscopy Atlas for the Coatings Industry in 1980 , recently revised in 1991 , and available as a spectral library. ${ }^{49,50}$ These resources provide spectra of pure, individual coating components such as pigments, fillers, and resins. However, the most comprehensive spectral database of automotive coating layers from actual samples is the Paint Data Query (PDQ), managed by the Royal Canadian Mounted Police. ${ }^{51}$ PDQ is a text-based search system with information on the color, layer sequence, and chemical composition of each layer as determined by FTIR spectroscopy. The organization collects over 1500 samples each year, over 500 of which are selected for inclusion in the database. ${ }^{52}$ However, the text-based system does not permit facile comparison of quantities of compounds present due to differences in collection parameters. This becomes an issue with clear coat analysis as all currently manufactured clear coats utilize either acrylic melamine styrene or urethane-modified acrylic melamine styrene as the binder. ${ }^{53}$ Direct comparison of spectra collected under identical parameters is essential to differentiating similar coatings from different sources. 
Most FTIR spectra in published databases is taken in transmission or attenuated total reflectance (ATR) modes. The merits of ATR over transmission mode have been discussed in the literature, including simpler sample preparation and increased intensity of minor peaks permitting discrimination of compounds present in small quantities. ${ }^{46,54}$ Transmission mode also typically requires time-consuming microtomy of thin sections to achieve sufficient transmission through opaque under coats. As FTIR spectroscopy detects functional groups, spectra collected in transmission and ATR modes should exhibit comparable peak locations. Unfortunately, the mode of FTIR spectroscopy has been shown to have significant effects on locations of major peaks by as much as $12 \mathrm{~cm}^{-1}$; this increases variation in knownquestioned scenarios if different methods are used to collect the spectra. ${ }^{55}$ Though analysis of clear coats in the literature is mixed on use of transmission and ATR, under coats are primarily analyzed in transmission mode as contamination between layers can occur with ATR on cross sections. Spectra in the current literature have been collected with a variety of sampling and instrument conditions since no standardized protocols for analysis of automotive coatings has been established. Similarly, no standard procedure for pigment identification in automotive base coats utilizing Raman spectroscopy has been determined.

Forensic examinations require analysis of samples in their entirety, which often include opaque under layers. Therefore, transmission FTIR spectroscopy will be used here, as all layers can be analyzed simultaneously and the disadvantages of ATR avoided. The goal is to develop recommendations for a protocol on forensic 
analyses of automotive coating fragments using transmission FTIR and Raman microspectroscopic methods.

\subsection{Experimental}

The sample set analyzed was previously discussed in Section 2.2 (Table 2.1). Samples for transmission FTIR microspectroscopy were prepared and analyzed in a low-pressure diamond anvil cell (DAC). Cross sections of $5 \mu \mathrm{m}$ thickness were microtomed from the epoxy-embedded samples, flattened with an Excel roller, and transferred to one diamond window of the DAC; the DAC was fully assembled with an O-ring and upper diamond window, and the cross section compressed. The upper window was removed and the cell reassembled so spectra were collected through only one diamond window to reduce noise from the absorbance of diamond. For Raman microspectroscopy, the epoxy-embedded samples were microtomed for surface smoothness, and spectra obtained directly on the embedded samples.

FTIR spectra were obtained on a Thermo Scientific Nicolet iN10 MX IR microscrope/spectrometer employing a KBr beamsplitter and liquid-nitrogen cooled MCT-A detector. The microscope component was a permanently aligned 15X (NA 0.7) objective. Spectra of $4 \mathrm{~cm}^{-1}$ resolution were collected in transmission mode from $500 \mathrm{~cm}^{-1}$ to $4000 \mathrm{~cm}^{-1}$ at three different locations for each layer. Though the lower limit of the detector provided by the manufacturer is $650 \mathrm{~cm}^{-1}$, some inorganic fillers exhibit intense, characteristic peaks in this region, so the range was extended and only large peaks in this region noted. Each spectrum was an average of 256 scans, and variable aperture was used to avoid interference from adjacent 
layers. Spectra of all visible layers were obtained with OMNIC Picta software in units of absorbance. Spectra were processed and analyzed with OriginLab software. The spectra of each layer were baseline corrected by subtracting the same baseline for the three locations. The spectra were then truncated to the fingerprint region of $675 \mathrm{~cm}^{-1}$ to $1800 \mathrm{~cm}^{-1}$ where the most useful peaks for binder and pigment identification occur, and absorbance normalized from 0 to 1 . Spectra were separated into five groups by layer type (clear coats, base coats, primer surfacers, primers, and substrates) before principal component analysis (PCA) was carried out with GNU Octave software.

Raman spectra were obtained with a Bruker Senterra spectrometer equipped with $532 \mathrm{~nm}$ and $785 \mathrm{~nm}$ lasers, and a Peltier-cooled silicon CCD detector. Only clear coats and base coats of the samples were analyzed as these layers exhibited the greatest spectral variation, and are most likely to be transferred in automotive collisions. Layers were examined using both lasers as different pigments used in base coats can exhibit preferential Raman scattering based on excitation source. ${ }^{43}$ A 20X (NA 0.40) objective was preferred for larger depth of field, resulting in a laser spot size of roughly $5 \mu \mathrm{m}$, but 50X (NA 0.45) and 100X (NA 0.90 ) objectives were employed if samples consisted of layers less than $10 \mu \mathrm{m}$ thick. ${ }^{56,57}$ The aperture size was kept constant at $50 \mu \mathrm{m}$ by $1000 \mu \mathrm{m}$. Power of the $532 \mathrm{~nm}$ laser was kept between $0.2 \mathrm{~mW}$ and $2 \mathrm{~mW}$ for most base coats and increased up to $10 \mathrm{~mW}$ for some clear coats. Power of the $785 \mathrm{~nm}$ laser was kept between $1 \mathrm{~mW}$ and $10 \mathrm{~mW}$ for base coats, and increased up to $100 \mathrm{~mW}$ for some clear coats. Sample degradation was prevented by adjusting the integration time 
and number of co-additions. In some cases, photobleaching was employed to decrease fluorescence; total sampling time was maintained under 3 minutes for the majority of spectra and under 5 minutes for all spectra. Spectra at three locations of each layer were collected with resolution of $9 \mathrm{~cm}^{-1}$ to $15 \mathrm{~cm}^{-1}$ utilizing a 400 lines/mm grating monochromator. The full spectral range for each laser was collected: $50 \mathrm{~cm}^{-1}$ to $4442 \mathrm{~cm}^{-1}$ for the $532 \mathrm{~nm}$ laser and $70 \mathrm{~cm}^{-1}$ to $3200 \mathrm{~cm}^{-1}$ for the $785 \mathrm{~nm}$ laser. Neon lamp calibration and background spectra were collected before analysis of each layer. Each spectrum was baseline corrected using Bruker OPUS software employing concave rubberband correction with 20 iterations. Further processing was performed with OriginLab software. Spectra obtained with the $532 \mathrm{~nm}$ and $785 \mathrm{~nm}$ lasers were truncated to $950 \mathrm{~cm}^{-1}$ to $1950 \mathrm{~cm}^{-1}$ and $550 \mathrm{~cm}^{-1}$ to $1850 \mathrm{~cm}^{-1}$, respectively. The Raman intensity of each spectrum was normalized from 0 to 1. PCA was performed on the clear coat spectra obtained with both lasers using GNU Octave software to identify major peaks and differences. Cl pigments were identified in the base coat spectra by comparison to literature spectra. ${ }^{58}$

\subsection{Results and Discussion}

The transmission FTIR spectra of the samples were used to identify the binder of each layer. These results are summarized in Table 3.2 for the clear coats and base coats, Table 3.3 for the primer surfacers and primers, and Table 3.4 for the substrates. The layers are abbreviated as follows: $\mathrm{C}=$ clear coat, $\mathrm{B}=$ base coat, $\mathrm{E}=$ effect layer, $\mathrm{PS}=$ primer surfacer, $\mathrm{P}=$ primer, and $\mathrm{S}=$ substrate. Only two samples contained effect layers separate from the base coat; these were grouped with the base coats. The number following the layer designation refers to 
the order of the layer in the sequence from substrate to surface for refinished samples; for instance, "18A C1" refers to the original clear coat of sample $18 \mathrm{~A}$ while "18A C2" refers to the refinish clear coat. The abbreviations for the structures identified are as follows: $\mathrm{A}=$ acrylic, $\mathrm{U}=$ urethane, $\mathrm{M}=$ melamine, $\mathrm{S}=$ styrene, $\mathrm{P}=$ polyester, $\mathrm{K}=$ alkyd, $\mathrm{E}=$ epoxy, $\mathrm{PE}-\mathrm{PP}=$ polyethylene-polypropylene copolymer, and $\mathrm{PU}=$ polyurethane. General structures of common binder components found in automotive coatings are provided for reference (Figure 2.1).<smiles>[R2]OC(=O)C([R17])(CC(C)C)C(C)(C)C</smiles><smiles>CCOC(=O)C(C)(C)C</smiles>

acrylic<smiles>[R2]C(COC(=O)[R]C(C)=O)OC</smiles>

$$
R_{2}=\text { fatty acid }
$$

polyester<smiles>CNC(=O)OC</smiles>

urethane

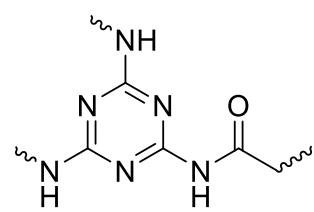

melamine

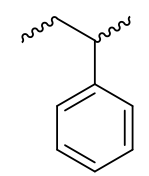

styrene

Figure 3.1 Structures of common binder components in automotive clear and base coats. 
Table 3.2 Binder Chemistry of Clear Coats and Base Coats Identified by IR Spectroscopy

\begin{tabular}{|c|c|c|c|c|}
\hline & Binder & & Binder & Additives \\
\hline $1 \mathrm{~A} \mathrm{C1}$ & A U M S (oxithane) & $1 \mathrm{AE} 1$ & $A \cup M S$ & \\
\hline $1 \mathrm{~A} C 2$ & A U M S (oxithane) & $1 \mathrm{~A} E 2$ & $A \cup M S$ & \\
\hline $2 \mathrm{~A} \mathrm{C1}$ & A U S (oxithane) & $2 A$ B1 & $\mathrm{A} \cup \mathrm{M}$ & \\
\hline $2 \mathrm{~A} \mathrm{C} 2$ & A U S (oxithane) & $2 A B 2$ & $A \cup M$ & \\
\hline $2 \mathrm{~A} \mathrm{C} 3$ & A U S (oxithane) & $2 A$ B3 & $A \cup M$ & \\
\hline $2 \mathrm{~A} \mathrm{C} 4$ & A U S (oxithane) & $2 \mathrm{~A} E 1$ & $A \cup M S$ & \\
\hline $3 \mathrm{C}$ & AS & $2 A$ E2 & $A \cup M S$ & \\
\hline $10 \mathrm{C}$ & A U S (oxithane) & $2 A$ E3 & $A \cup M S$ & \\
\hline $10 P \mathrm{C}$ & A U M S & $3 \mathrm{~B}$ & A S & \\
\hline $11 \mathrm{C} 1$ & A M & $10 \mathrm{~B}$ & $A \cup S$ & \\
\hline $11 \mathrm{C} 2$ & A U S (oxithane) & 10P B & $A \cup M$ & \\
\hline $12 \mathrm{C} 1$ & AMS & $11 \mathrm{~B} 1$ & A M & \\
\hline $12 \mathrm{C} 2$ & A U S (oxithane) & $11 \mathrm{~B} 2$ & A U S (oxithane) & \\
\hline $13 \mathrm{C} 1$ & A U S (oxithane) & $12 \mathrm{~B} 1$ & $A \cup M$ & \\
\hline $13 \mathrm{C} 2$ & A U S (oxithane) & $12 \mathrm{~B} 2$ & $A \cup M S$ & \\
\hline $13 \mathrm{C} 3$ & A U S (oxithane) & $13 \mathrm{~B} 1$ & $A \cup M S$ & \\
\hline $15 \mathrm{C}$ & A S & $13 \mathrm{~B} 2$ & A U S & \\
\hline $17 \mathrm{C}$ & $A \cup S$ & $15 \mathrm{~B}$ & $\mathrm{AM}$ & $\mathrm{BaSO}_{4}$ \\
\hline $18 \mathrm{C} 1$ & $\mathrm{AM}$ & $17 \mathrm{~B}$ & K & \\
\hline $18 \mathrm{C} 2$ & A M & $18 \mathrm{~B} 1$ & $A \cup M$ & \\
\hline $18 \mathrm{~A} \mathrm{C} 1$ & A U M S & 18 B2 & $A \cup M$ & \\
\hline 18A C2 & A U S (oxithane) & 18A B1 & $A \cup M$ & \\
\hline $19 A C$ & A U S (oxithane) & 18A B2 & A K U & \\
\hline 34PS C & $A \cup M S$ & $19 A B$ & A K U & \\
\hline $34 \mathrm{PW} \mathrm{C}$ & $A \cup M S$ & $34 P S B$ & $A \cup M S$ & \\
\hline $58 \mathrm{C}$ & A M & 34PW B & $A \cup M$ & \\
\hline $58 \mathrm{P} \mathrm{C}$ & $A M$ & $58 \mathrm{~B}$ & A M & $\mathrm{TiO}_{2}$ \\
\hline $59 \mathrm{C} 1$ & A M & $58 \mathrm{P} B$ & A M & $\mathrm{TiO}_{2}$ \\
\hline $59 \mathrm{C} 2$ & A M & 59 B1 & A M & $\mathrm{TiO}_{2}$ \\
\hline D782 C & A U S (oxithane) & 59 B2 & $\mathrm{A} M$ & $\mathrm{TiO}_{2}$ \\
\hline \multirow[t]{2}{*}{ D786 C } & $A M$ & D782 B & $A \cup S$ & \\
\hline & & D786 B & $\mathrm{A} M$ & $\mathrm{BaSO}_{4}$ \\
\hline
\end{tabular}


Table 3.3 Binder Chemistries of Primers Identified by IR Spectroscopy

\begin{tabular}{|c|c|c|c|c|c|}
\hline & Binder & Additives & & Binder & Additives \\
\hline 1A PS & $A \cup M$ & $\mathrm{TiO}_{2}, \mathrm{BaSO}_{4}$ & $1 \mathrm{AP}$ & $E$ & \\
\hline 2A PS & $A \cup M$ & & $2 \mathrm{AP}$ & $A \cup M$ & \\
\hline 3 PS & A M & $\mathrm{TiO}_{2}$ & $3 P$ & E & $\mathrm{TiO}_{2}$ \\
\hline 10P PS & A M & & $10 P$ & $E$ & \\
\hline 12 PS & P M & $\mathrm{TiO}_{2}$ & $10 \mathrm{P} P$ & $E$ & \\
\hline $15 \mathrm{PS}$ & A M & $\mathrm{BaSO}_{4}$ & $11 \mathrm{P} 1$ & A M & \\
\hline $18 \mathrm{PS}$ & A M & $\mathrm{BaSO}_{4}$ & $11 \mathrm{P} 2$ & A M & \\
\hline 18A PS & P M & $\mathrm{TiO}_{2}, \mathrm{BaSO}_{4}$ & $12 \mathrm{P}$ & $E$ & $\mathrm{TiO}_{2}$ \\
\hline 34PS PS & A M & $\mathrm{TiO}_{2}$ & $13 P$ & $E$ & $\mathrm{CaCO}_{3}, \mathrm{BaSO}_{4}$ \\
\hline $34 \mathrm{~W}$ PS & A M & $\mathrm{TiO}_{2}$ & $15 P$ & $E$ & $\mathrm{BaSO}_{4}$ \\
\hline 58 PS & P M & $\mathrm{TiO}_{2}$ & $17 \mathrm{P}$ & $\mathrm{K}$ & $\mathrm{BaSO}_{4}$ \\
\hline 58P PS & $E$ & & $18 \mathrm{P}$ & $E$ & \\
\hline \multirow[t]{10}{*}{ D786 PS } & P M & & $18 \mathrm{~A} \mathrm{P}$ & $E$ & \\
\hline & & & $19 A P$ & A & $\mathrm{CaCO}_{3}, \mathrm{BaSO}_{4}$ \\
\hline & & & 34PS P & E & $\mathrm{BaSO}_{4}$ \\
\hline & & & 34PW P & $E$ & $\mathrm{BaSO}_{4}$ \\
\hline & & & $58 \mathrm{P}$ & $E$ & \\
\hline & & & $58 \mathrm{P} P$ & $E$ & \\
\hline & & & 59 P1 & A M & \\
\hline & & & 59 P2 & A M & \\
\hline & & & D782 P & $A \cup S$ & \\
\hline & & & D786 P & E & \\
\hline
\end{tabular}

Table 3.4 Composition of Substrates Determined by IR Spectroscopy

\begin{tabular}{l|cc} 
& Binder & Additives \\
\hline $2 \mathrm{~A} \mathrm{~S}$ & PE-PP & talc \\
$11 \mathrm{~S}$ & PU & \\
$13 \mathrm{~S}$ & PE-PP & \\
$17 \mathrm{~S}$ & PE-PP & \\
$19 \mathrm{~A} \mathrm{~S}$ & PE-PP & talc \\
$59 \mathrm{~S}$ & PE-PP & talc \\
D782 S & PE-PP & talc
\end{tabular}

The binders of automotive clear coats are generally some form of acrylic melamine, though urethane modification is not unusual to impart higher gloss, hardness, and ruggedness. ${ }^{10} \mathrm{~A}$ new generation of "oxithane-type" urethane-modi- 
fied acrylic clear coat is being employed in more refinishes with a distinct IR spectrum present in this sample set (Figure 3.2). ${ }^{10}$ This type of binder was identified by the doublet of nearly equal intensity at roughly $1690 \mathrm{~cm}^{-1}$ and $1730 \mathrm{~cm}^{-1}$, and the sharp peak at roughly $1463 \mathrm{~cm}^{-1}$ of similar intensity. The peak at $1730 \mathrm{~cm}^{-1}$ corresponds to the carbonyl stretching vibration common to all the components in automotive clear coats. The peak at $1690 \mathrm{~cm}^{-1}$ corresponds specifically to the carbamate carbon-oxygen single bond stretching vibration of the urethane component. The $1463 \mathrm{~cm}^{-1}$ peak corresponds to methyl and methylene deformation vibrations in the acrylic backbone. ${ }^{10}$ The presence of melamine as a crosslinking agent in many acrylic clear coats was noted by the characteristic peaks at $815 \mathrm{~cm}^{-1}$ and $1550 \mathrm{~cm}^{-1}$ (Figure 3.3). The $815 \mathrm{~cm}^{-1}$ peak corresponds to the triazine ring carbonhydrogen bending vibration while the $1550 \mathrm{~cm}^{-1}$ peak is due to in-plane vibration of the triazine ring. ${ }^{46}$ Many acrylic clear coats are also styrene-modified, resulting in sharp peaks at $700 \mathrm{~cm}^{-1}$ and $760 \mathrm{~cm}^{-1}$ from the aromatic carbon-carbon double bond bending vibrations (Figure 3.3). Though the example spectra provided are of clear coats, urethane, melamine, and styrene modifications were observed in a number of the base coats spectra as well (see Table 3.2). 


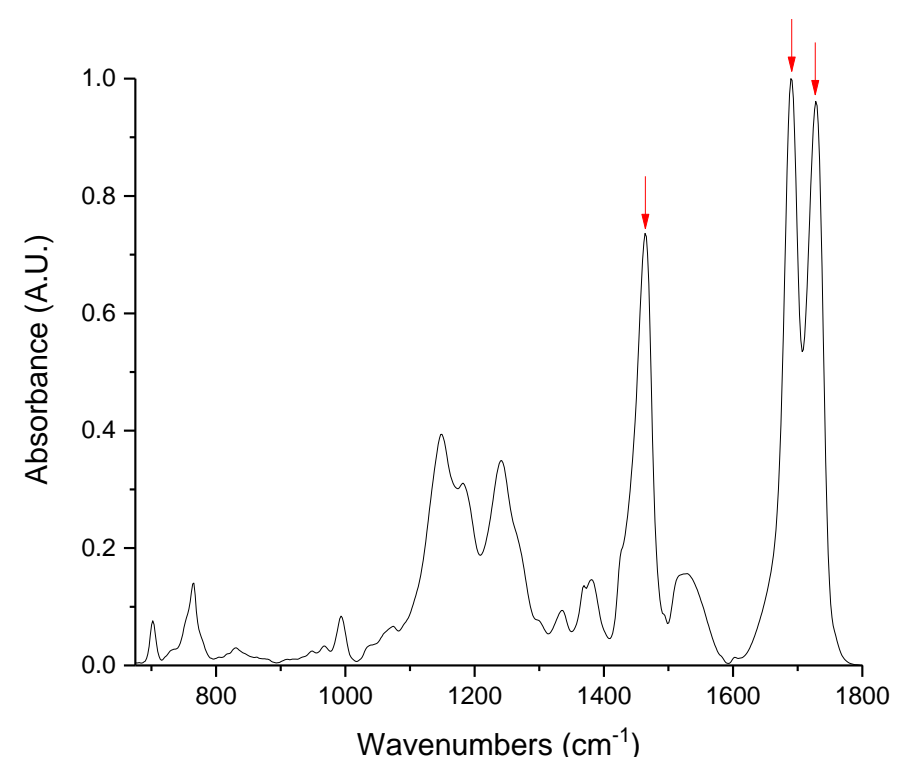

Figure 3.2 FTIR spectrum of the refinish clear coat of sample 11, an "oxithanetype" urethane-modified acrylic binder. Arrows represent key peaks.

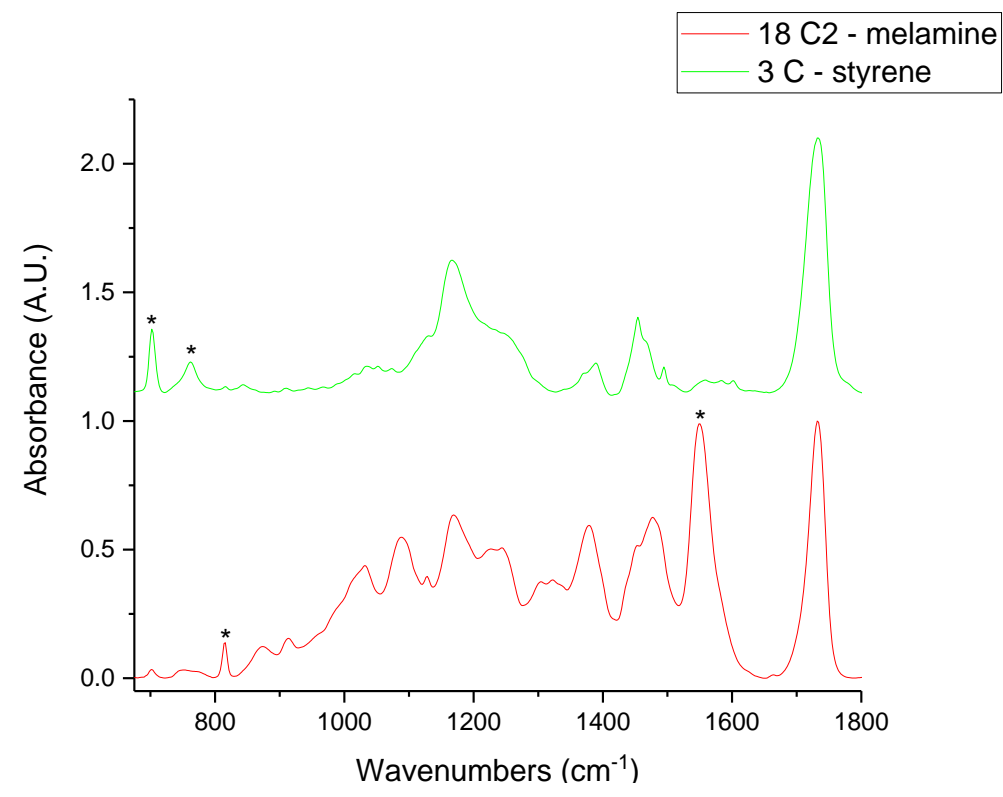

Figure 3.3 FTIR spectrum of acrylic clear coats with melamine and styrene modifications. Asterisks represent key peaks.

The primary binder chemistries of automotive base coats and clear coats can be broadly classified as either acrylic, alkyd, or polyester, as these have been used by both domestic and international manufacturers in recent history (see 
Table 3.1$) \cdot{ }^{10}$ As the main functional groups of these binders are all very similar (ester-based), the fingerprint region between $1100 \mathrm{~cm}^{-1}$ and $1300 \mathrm{~cm}^{-1}$ is extremely important in differentiating these binders. If the highest intensity peak in this region lies between $1150 \mathrm{~cm}^{-1}$ and $1180 \mathrm{~cm}^{-1}$, the binder is likely acrylic. If this peak is between $1235 \mathrm{~cm}^{-1}$ and $1245 \mathrm{~cm}^{-1}$, the binder is likely polyester, and if this peak is between $1260 \mathrm{~cm}^{-1}$ and $1280 \mathrm{~cm}^{-1}$, the binder is likely alkyd-based. ${ }^{10}$ This method was based on examination of known coating spectra for general trends in major absorptions. These binder types were encountered in the present sample set (Figure 3.4). The clear coat of sample 15 was acrylic with the key peak at $1165 \mathrm{~cm}^{-1}$. The base coat of sample 17 was characterized as alkyd-based with the highest intensity peak in this region at $1265 \mathrm{~cm}^{-1}$. Finally, the spectra of the primer surfacer of sample 12 exhibited the highest intensity peak in this region at $1238 \mathrm{~cm}^{-1}$. 


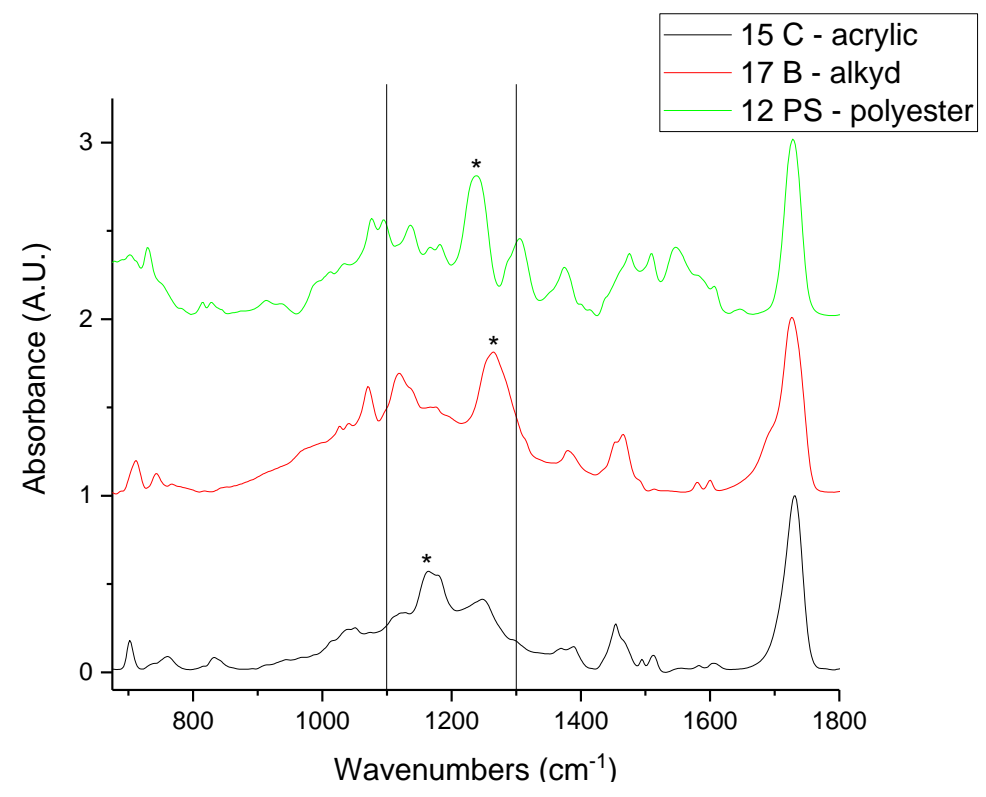

Figure 3.4 FTIR spectra of acrylic, alkyd, and polyester coating layers. Vertical lines delineate the region of interest for differentiation between these binder types; asterisks represent the highest intensity peak in this region.

Epoxy was not observed as a binder in any of the clear coats and base coats examined. This was expected given the history of clear coat chemistries employed by automotive manufacturers. However, epoxy was observed as the main chemistry for primers in this sample set. The FTIR spectrum of epoxy is easily recognized and differentiated from that of the previously mentioned binders because it is not ester-based and varies only slightly between samples. However, the $1730 \mathrm{~cm}^{-1}$ ester carbonyl peak can be present in spectra of many alkyd or acrylicmodified epoxy primers. ${ }^{10}$ The FTIR spectra of epoxy primers exhibit characteristic peaks at $830 \mathrm{~cm}^{-1}$ and $1510 \mathrm{~cm}^{-1}$ (Figure 3.5). The peak at $830 \mathrm{~cm}^{-1}$ is the result of out-of-plane bending of adjacent hydrogens in bisphenol A, while the peak at $1510 \mathrm{~cm}^{-1}$ is the carbon-carbon double bond stretch of the bisphenol aromatic ring. ${ }^{10}$ Due to the intense characteristic epoxy bands in the $1100 \mathrm{~cm}^{-1}$ to $1300 \mathrm{~cm}^{-}$ 
1 region, it is difficult to determine what other resin chemistries (acrylic or alkyd) are present if in small quantities. In the present sample set, the only non-primer epoxy resin identified was the primer surfacer of sample 58P. This standard panel corresponds to sample 58, which had a polyester primer surfacer. However, the primer surfacers of the other three standard samples examined (10P, 34PS, and 34PW) did not match the primer surfacers of their corresponding samples (10 and 34) either.

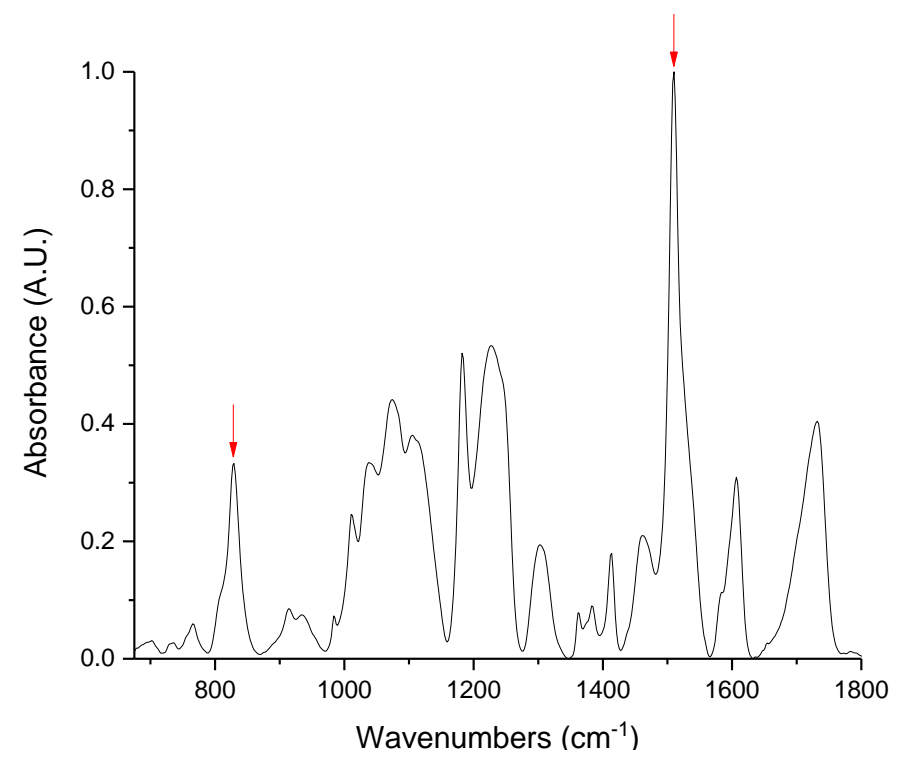

Figure 3.5 FTIR spectrum of the epoxy primer of sample 1A. Arrows represent key peaks for epoxy identification.

Finally, the substrates of the samples on plastic were analyzed to see if any differentiating information could be gained. The primary chemistry observed was polyethylene-polypropylene copolymer, with only sample 11 having a polyurethane substrate. The characteristic peaks for PE-PP occur at $1014 \mathrm{~cm}^{-1}, 1377 \mathrm{~cm}^{-1}$, and $1460 \mathrm{~cm}^{-1}$ (Figure 3.6). The primary peaks for PU occur at $1103 \mathrm{~cm}^{-1}$ and 
$1539 \mathrm{~cm}^{-1}$, based solely on the substrate of sample 11 when compared to reference spectra available in spectral libraries.

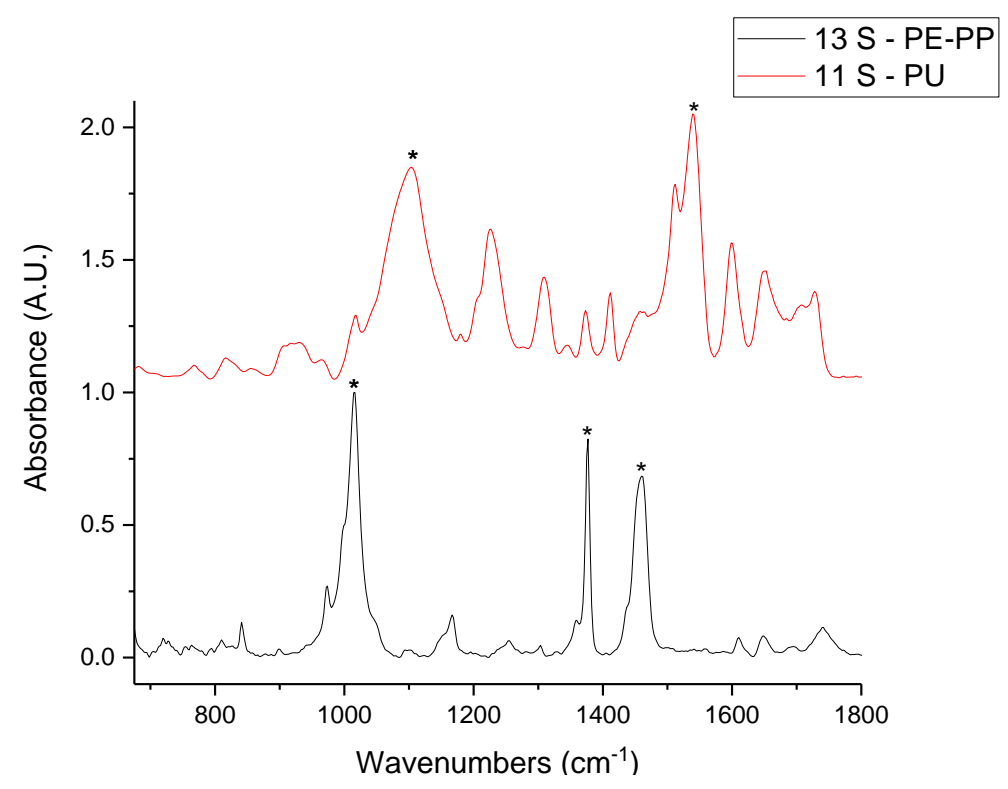

Figure 3.6 FTIR spectrum of the substrates of samples 11 (PU) and 13 (PE-PP). Asterisks represent key peaks for identification of these substrate chemistries.

As previously mentioned, FTIR spectroscopy is a poor method for detecting pigments as the binder routinely exhibits intense absorbance, obscuring any characteristic peaks. However, some inorganic fillers commonly employed in the under layers of automotive coatings exhibited peaks of sufficient intensity for identification. These included titanium dioxide in all the white base coats, barium sulfate, calcium carbonate, and talc in some of the substrates; this indicates FTIR spectroscopy of substrates may provide useful differentiating information based on inorganic filler presence. Titanium dioxide exhibits a characteristic broad peak centered at roughly $640 \mathrm{~cm}^{-1}$ as observed in the spectrum of the base coat of sample 58P (Figure 3.7). Barium sulfate exhibits characteristic peaks at roughly $611 \mathrm{~cm}^{-1}$ and $638 \mathrm{~cm}^{-1}$, visible in the spectrum of the primer of sample 19A. Also present in 
this layer was calcium carbonate which exhibits distinct peaks at $875 \mathrm{~cm}^{-1}$ and $1454 \mathrm{~cm}^{-1}$. Finally, many of the PE-PP substrates contained talc, which exhibits a distinct peak at $1022 \mathrm{~cm}^{-1}$.

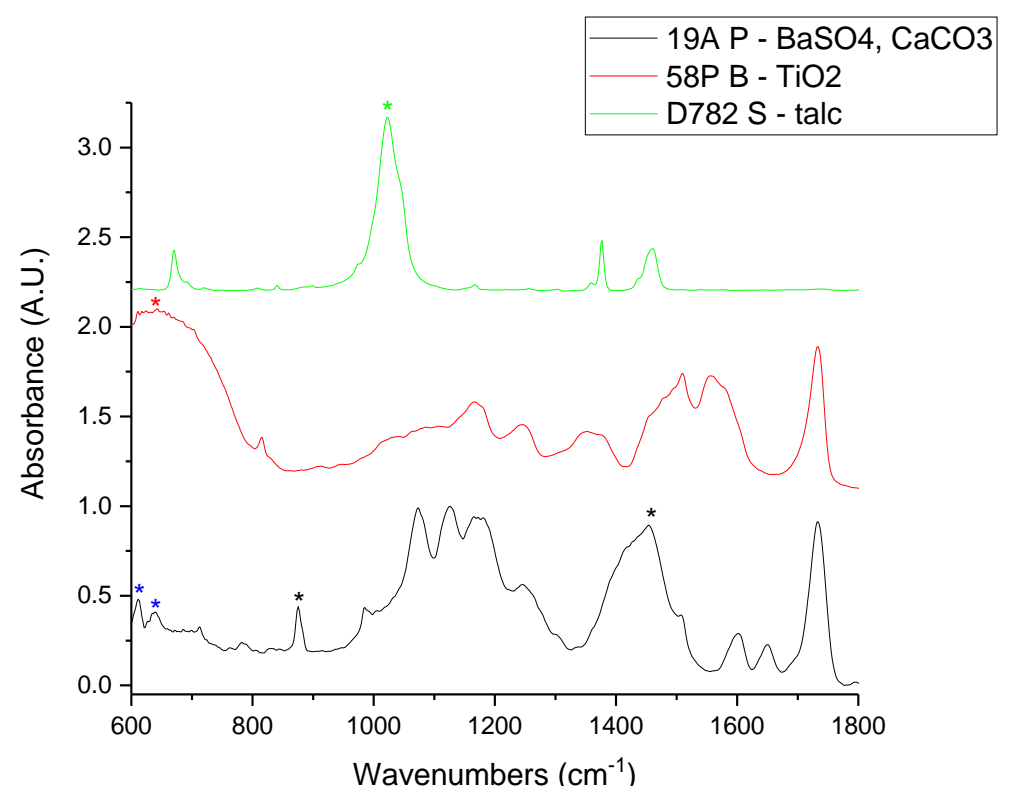

Figure 3.7 FTIR spectra of automotive coating layers containing barium sulfate (black), calcium carbonate (blue), titanium dioxide (red), and talc (green). Asterisks represent the key peaks for identifying these additives in IR spectra.

The binders of the examined clear coats and base coats were primarily acrylic with urethane, melamine, and styrene modifications. The primer surfacers were primarily acrylic-melamine with some polyester-melamine layers identified, and the primers were nearly all epoxy. Though the present sample set was too small to obtain quantitative data for make or model identification from principal component analysis, the score plots of the spectra grouped by layer type were observed to identify any significantly different spectra. Score plots are visual representations of the data in the new coordinate system. In the score plot of the first 
two principal components (PCs) of the clear coat spectra, all of the samples were fairly scattered (Figure 3.8). The factor loadings of PC 1 indicated the main differentiating features observed in this spectral set were at $815 \mathrm{~cm}^{-1}, 1487 \mathrm{~cm}^{-1}$, and $1550 \mathrm{~cm}^{-1}$, characteristic of melamine; this indicated the primary differentiating factor for the clear coats was melamine-modification (Figure 3.9). The score plot of the base coat set were scattered, but a large cluster of samples with positive PC 2 correlation emerged (Figure 3.10). The factor loadings for PC 2 indicated the samples with negative PC 2 differed primarily in having a large, broad peak in the $675 \mathrm{~cm}^{-1}$ region; this is the characteristic titanium dioxide peak, so this was the group of white samples (Figure 3.11). The primer surfacer set did not yield any clusters based on the new coordinate system. However, a group exhibiting positive correlation with PC 1 was evident from the score plot of the primer spectra set (Figure 3.12). This group had significant peaks at $830 \mathrm{~cm}^{-1}$ and $1510 \mathrm{~cm}^{-1}$ based on the factor loadings for PC 1, which are characteristic of epoxy; this separated the primer set into two groups of epoxy-based or other (Figure 3.13). The substrate set was previously grouped by observation into PE-PP and PU substrates; this was illustrated by the score plot in which PC 1 corresponded to the PU substrate of sample 11 (Figure 3.14). The PC 1 factor loadings confirmed this observation, as the positive correlations were basically the spectrum of the PU substrate. The group with negative PC 2 was the PE-PP substrates lacking talc based on the 1024 $\mathrm{cm}^{-1}$ peak in the factor loadings. 


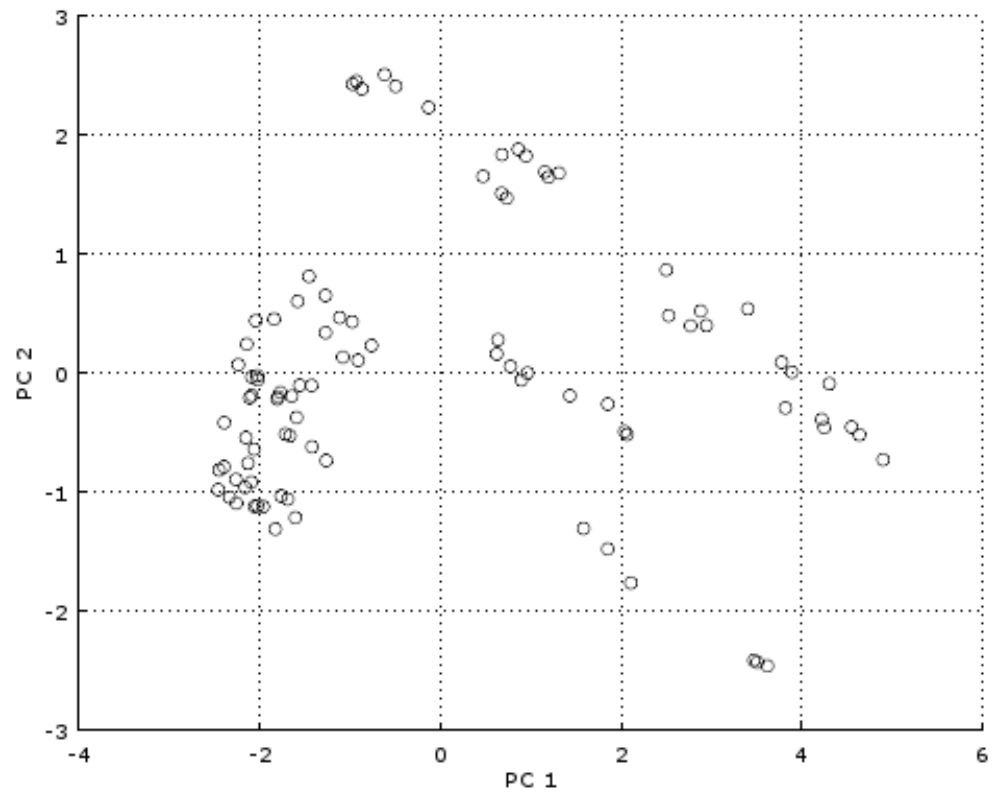

Figure 3.8 Score plot for PCs 1 and 2 of clear coat IR spectra set.

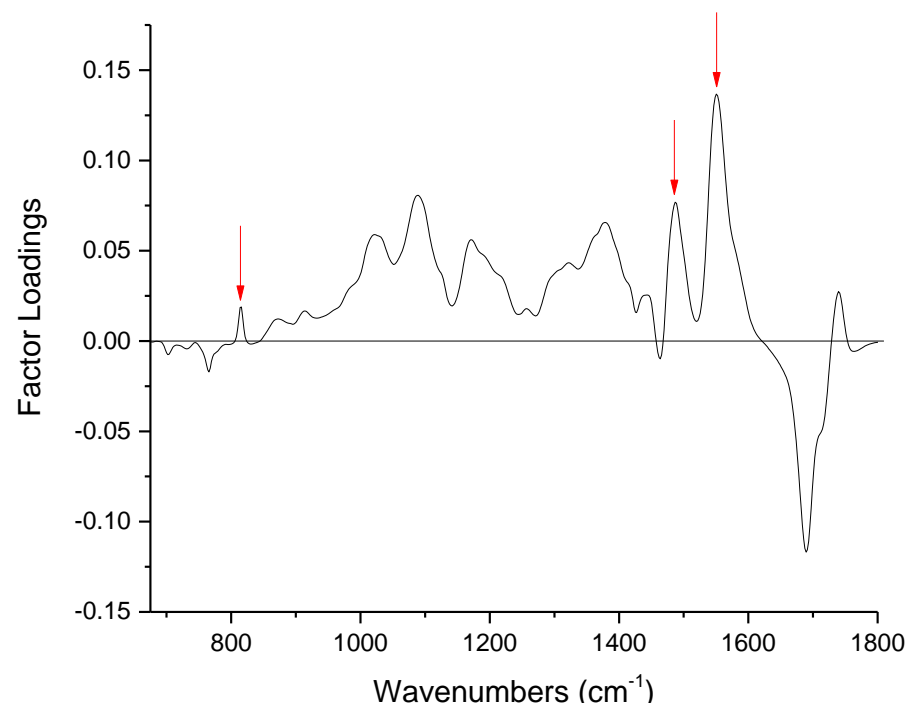

Figure 3.9 Factor loadings for PC 1 of the clear coat FTIR spectra set. Arrows represent peaks corresponding to melamine modification. 


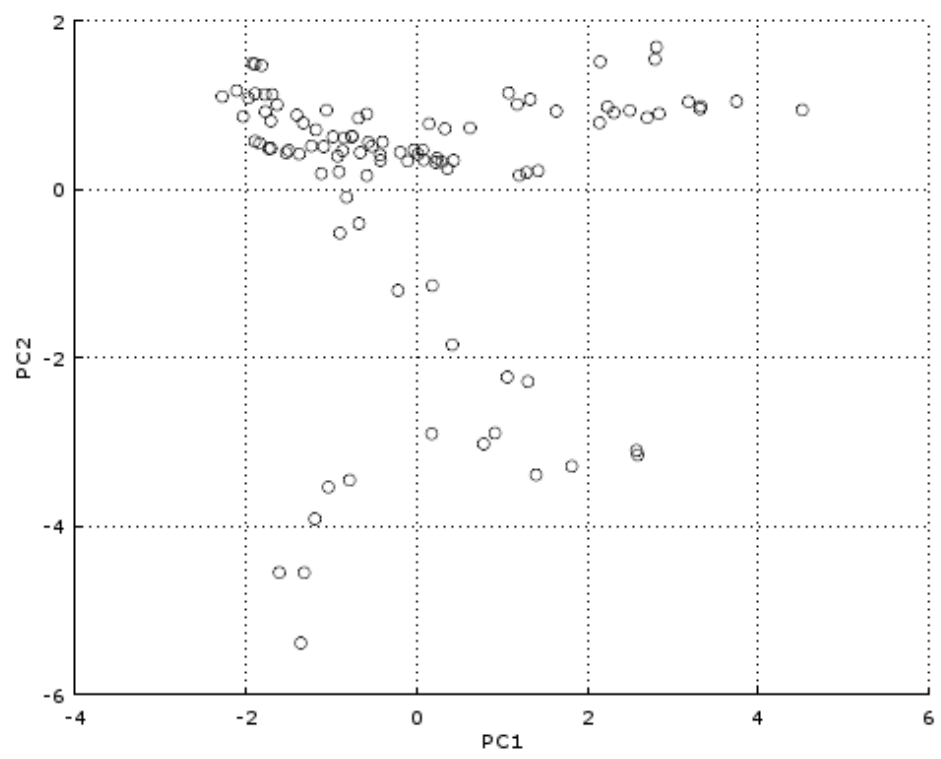

Figure 3.10 Score plot of PCs 1 and 2 of base coat IR spectra set.

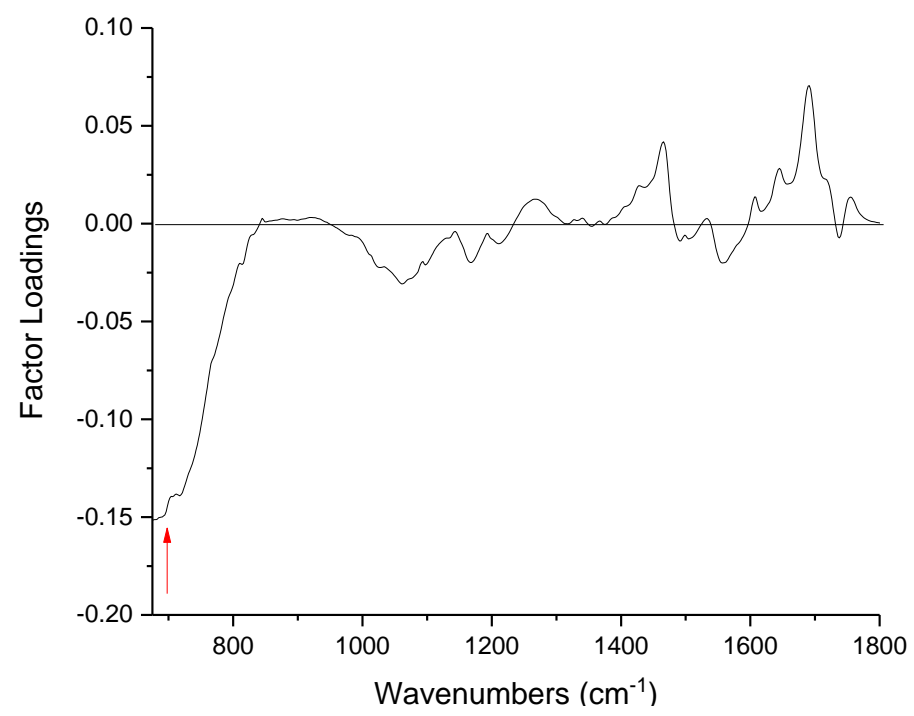

Figure 3.11 Factor loadings for PC 2 of the base coat FTIR spectra set. Arrow represents the peak corresponding to titanium dioxide. 


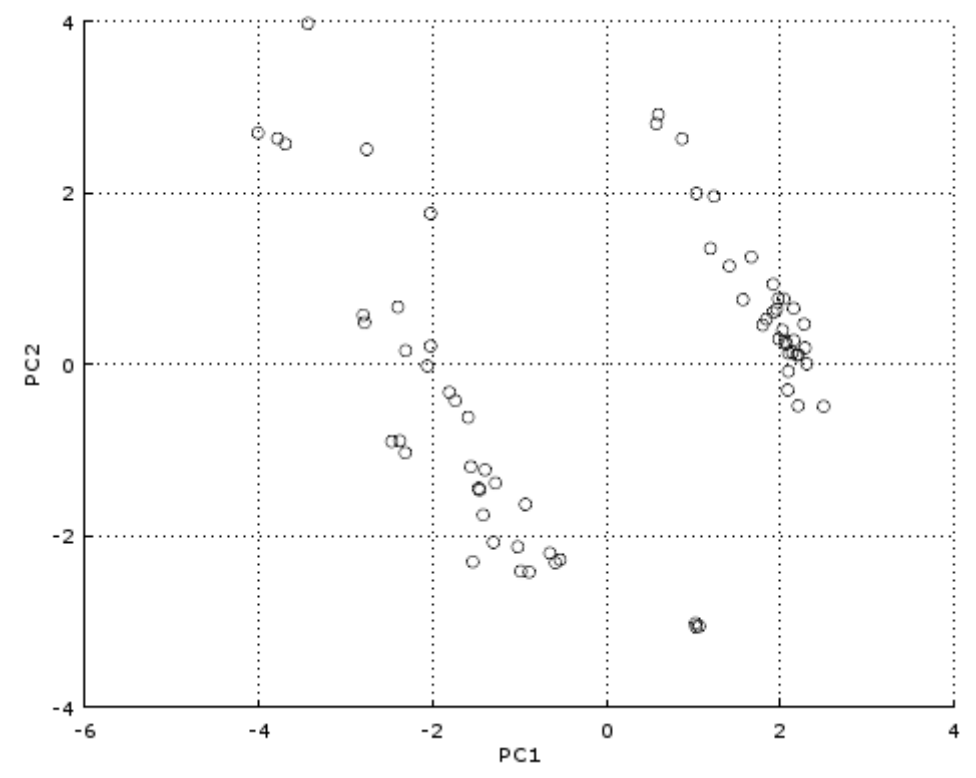

Figure 3.12 Score plots for PCs 1 and 2 of primer IR spectra set.

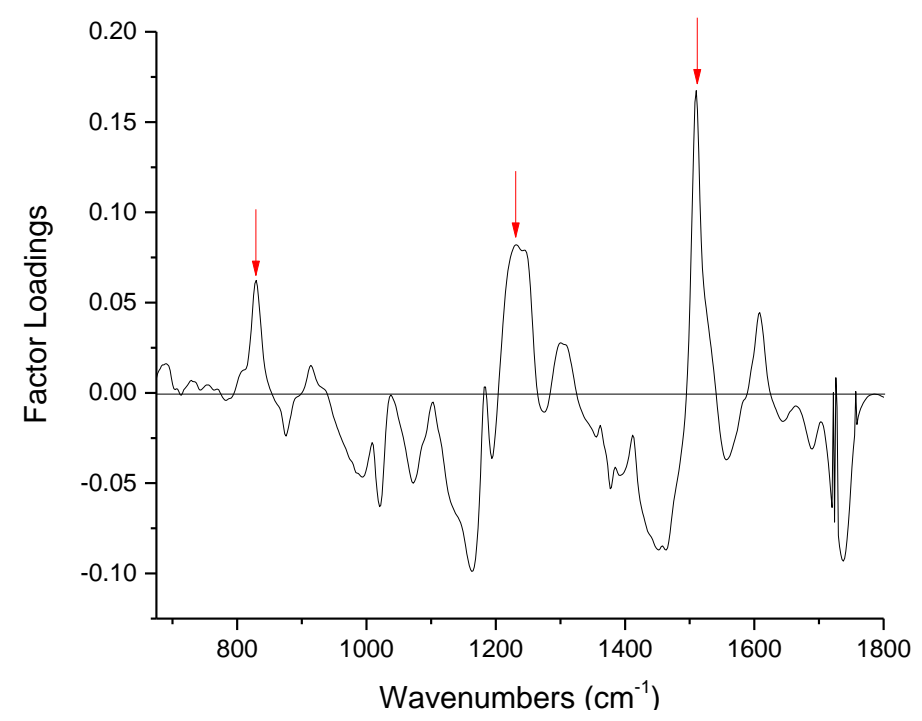

Figure 3.13 Factor loadings for PC 1 of the primer FTIR spectra set. Arrows represent peaks indicating presence of epoxy. 

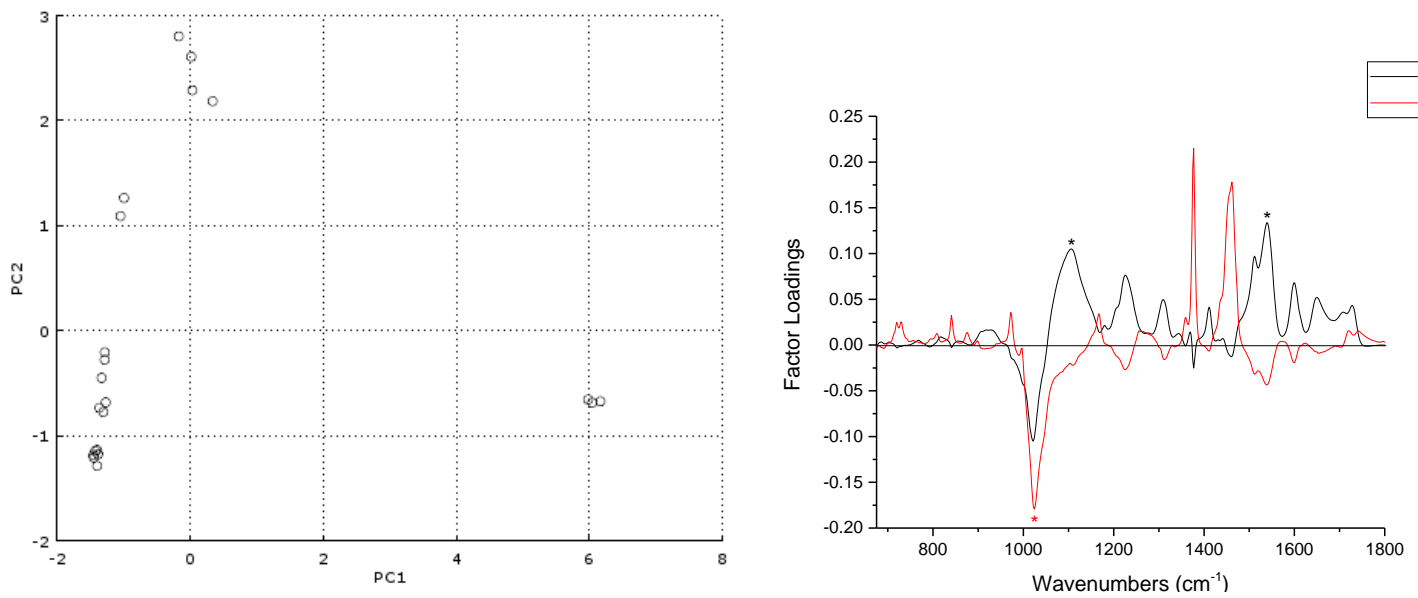

Figure 3.14 Score plots for PCs 1 and 2 of substrate IR spectra set (left). The cluster on the left represents the substrate of sample 11. Factor loadings for PCs 1 and 2 of the substrate FTIR spectra set (right). The asterisks indicate key peaks corresponding to PU in PC 1 and talc in PC 2.

The Raman spectroscopy results for the clear and base coats are summarized in Table 3.5. In the clear coat portion, "+" indicates spectra with relatively low or no fluorescence background were obtained, "-" indicates spectra with some fluorescence, but significant peaks were obtained, and " $x$ " indicates the sample exhibited only fluorescence with no significant peaks. Raman spectra of 14 clear coats were obtained with the $532 \mathrm{~nm}$ laser and 20 with the $785 \mathrm{~nm}$ laser. This was expected as near-IR lasers have been shown to induce less fluorescence in the literature..$^{43,59}$ The pigments identified in the base coat spectra are noted with their Cl name. 
Table 3.5 Raman Spectroscopy Results

\begin{tabular}{|c|c|c|c|c|c|}
\hline Sample & $532 \mathrm{~nm}$ & $785 \mathrm{~nm}$ & Sample & $532 \mathrm{~nm}$ & $785 \mathrm{~nm}$ \\
\hline $1 \mathrm{~A} \mathrm{C1}$ & + & $x$ & $1 \mathrm{~A} \mathrm{E} 1$ & PB60, fluorescence & fluorescence \\
\hline $1 \mathrm{~A} \mathrm{C} 2$ & - & $x$ & $1 \mathrm{~A} E 2$ & PB60 & fluorescence \\
\hline $2 \mathrm{~A} \mathrm{C1}$ & - & $x$ & $2 A B 1$ & fluorescence & PW6, fluorescence \\
\hline 2A C2 & - & $x$ & $2 A B 2$ & fluorescence & PW6, fluorescence \\
\hline $2 \mathrm{~A} \mathrm{C} 3$ & - & $x$ & $2 A B 3$ & fluorescence & PW6, fluorescence \\
\hline $2 \mathrm{~A} \mathrm{C} 4$ & - & + & $2 \mathrm{~A} E 1$ & fluorescence & fluorescence \\
\hline $3 \mathrm{C}$ & $x$ & + & $2 A E 2$ & fluorescence & fluorescence \\
\hline $10 \mathrm{C}$ & + & + & 2A E3 & fluorescence & fluorescence \\
\hline $10 \mathrm{P} C$ & $x$ & + & $3 \mathrm{~B}$ & fluorescence & PR254(a) \\
\hline $11 \mathrm{C} 1$ & $x$ & $x$ & $10 \mathrm{~B}$ & PBK6, fluorescence & PBK6, fluorescence \\
\hline $11 \mathrm{C} 2$ & + & + & $10 P B$ & fluorescence & PBK7 \\
\hline $12 \mathrm{C} 1$ & $x$ & + & $11 \mathrm{~B} 1$ & fluorescence & PB60 \\
\hline $12 \mathrm{C} 2$ & $x$ & + & $11 \mathrm{~B} 2$ & PBK6 & PBK6 \\
\hline $13 \mathrm{C} 1$ & $x$ & + & $12 \mathrm{~B} 1$ & fluorescence & PR254(a) \\
\hline $13 \mathrm{C} 2$ & $x$ & + & $12 \mathrm{~B} 2$ & fluorescence & PR254(a) \\
\hline $13 \mathrm{C3}$ & $x$ & + & $13 \mathrm{~B} 1$ & fluorescence & PR254(a) \\
\hline $15 \mathrm{C}$ & $x$ & - & $13 \mathrm{~B} 2$ & fluorescence & PR254(a) \\
\hline $17 \mathrm{C}$ & $x$ & + & $15 B$ & PBK7, fluorescence & PBK7 \\
\hline $18 \mathrm{C} 1$ & $x$ & $x$ & $17 \mathrm{~B}$ & fluorescence & PBK9 \\
\hline 18 C2 & $x$ & + & $18 \mathrm{~B} 1$ & PR149, fluorescence & fluorescence \\
\hline $18 \mathrm{~A} \mathrm{C1}$ & + & $x$ & 18 B2 & PR149, fluorescence & fluorescence \\
\hline 18A C2 & + & + & $18 \mathrm{~A} \mathrm{~B} 1$ & PB60 & PB60 \\
\hline $19 A C$ & $x$ & + & 18A B2 & PB15, PV23( $\beta)$ & PB15, PV23( $\beta)$, fluorescence \\
\hline 34PS C & $x$ & $x$ & $19 A B$ & PV23(ß), fluorescence & PB15, PB60 \\
\hline 34PW C & $x$ & $x$ & 34PS B & PB60 & PB60, fluorescence \\
\hline $58 \mathrm{C}$ & - & + & 34PW B & PB60 & PB60, fluorescence \\
\hline $58 \mathrm{P} \mathrm{C}$ & - & - & $58 \mathrm{~B}$ & PW6, fluorescence & PW6 \\
\hline 59 C1 & $x$ & - & $58 \mathrm{P} B$ & PW6 & PW6, fluorescence \\
\hline 59 C2 & $x$ & - & 59 B1 & PW6 & PW6 \\
\hline D782 C & - & + & 59 B2 & PW6 & PW6 \\
\hline D786 C & - & $x$ & $\begin{array}{l}\text { D782 B } \\
\text { D786 B }\end{array}$ & $\begin{array}{l}\text { PV23 } \\
\text { fluorescence }\end{array}$ & $\begin{array}{l}\text { PB15, fluorescence } \\
\text { fluorescence }\end{array}$ \\
\hline
\end{tabular}

Since the clear coat binders were already identified by IR spectroscopy, PCA was done on the spectral sets collected with the two lasers to determine if any significant differences useful for differentiation existed between the Raman spectra. An important consideration in analysis of these spectra was how the $532 \mathrm{~nm}$ and $785 \mathrm{~nm}$ lasers necessarily produced different photons even if the laser powers were comparable. Raman shift is still relative to the laser used as opposed 
to the absolute frequency measurement in IR spectroscopy. ${ }^{60}$ The Raman shift ranges of common functional groups found in the literature are fairly wide, and the vibrations labeled here differed slightly depending on the dataset.

The factor loadings for the first three PCs of the clear coat Raman spectral set collected with the $532 \mathrm{~nm}$ laser exhibited highest intensity positive correlations for PC 1 at $1128 \mathrm{~cm}^{-1}, 1407 \mathrm{~cm}^{-1}$, and $1623 \mathrm{~cm}^{-1}$ (Figure 3.15). This first vibration corresponds to the acyclic aliphatic carbon-carbon chain bending, a general vibration of the acrylic backbone. The second vibration at $1400 \mathrm{~cm}^{-1}$ corresponds to the asymmetric methyl and $\mathrm{CH}_{2}$ stretching, another result of the acrylic backbone. The vibration at $1623 \mathrm{~cm}^{-1}$ corresponds to a carbon-nitrogen double bond vibration, likely the result of melamine modification and in agreement with the IR spectroscopy clear coat analysis. The high intensity correlations for PC 2 occurred at Raman shifts similar to PC 1 , but with a negative factor loading at roughly $1623 \mathrm{~cm}^{-1}$ representing samples lacking melamine modification. The PC 3 positive loadings at roughly $1020 \mathrm{~cm}^{-1}$ and $1048 \mathrm{~cm}^{-1}$ were most intense, and likely the result of styrene modification as the trigonal ring breathing of the aromatic ring manifests as a peak at roughly $1031 \mathrm{~cm}^{-1}$. 


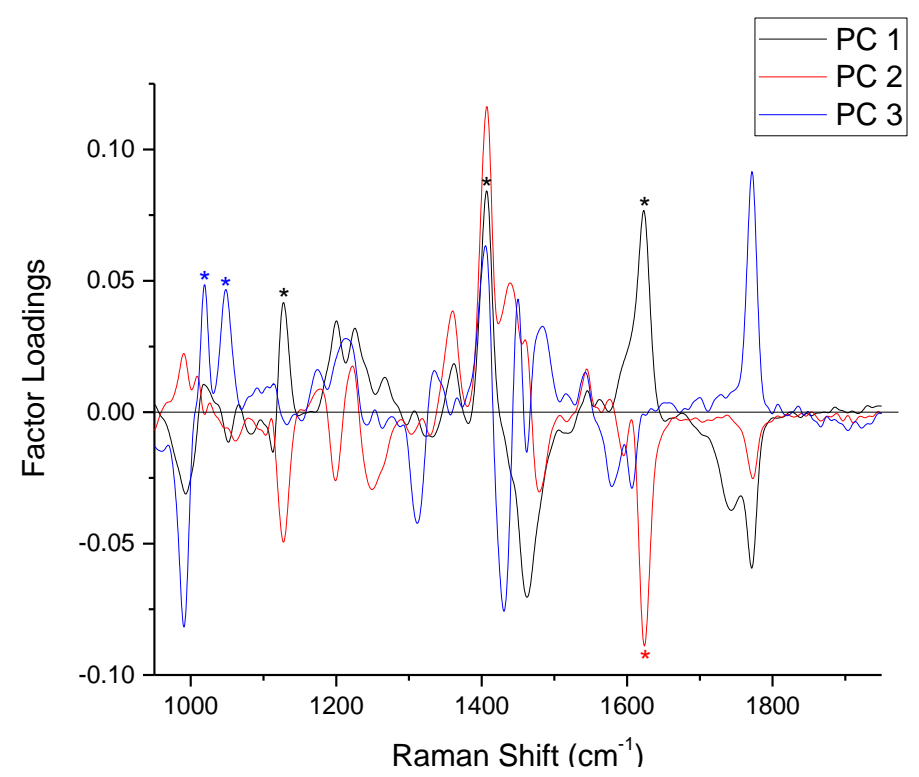

Figure 3.15 Factor loadings of the first three PCs for clear coat Raman spectra taken with the $532 \mathrm{~nm}$ laser. Asterisks identify the highest intensity peaks in the factor loadings of each PC.

The factor loadings for the first three PCs of the clear coat Raman spectra collected with the $785 \mathrm{~nm}$ laser are shown in Figure 3.16. The factor loadings for PC 1 were primarily negative with significant loadings at $978 \mathrm{~cm}^{-1}, 1303 \mathrm{~cm}^{-1}$, $1450 \mathrm{~cm}^{-1}, 1595 \mathrm{~cm}^{-1}$, and $1732 \mathrm{~cm}^{-1}$. The $978 \mathrm{~cm}^{-1}$ peak is the result of triazine ring breathing, indicative of melamine modification. The $1303 \mathrm{~cm}^{-1}$ peak is due to $\mathrm{CH}_{2}$ in-phase twisting vibrations from the acrylic backbone. The $1450 \mathrm{~cm}^{-1}$ peak is also due to the acrylic binder and the result of methyl and $\mathrm{CH}_{2}$ deformations in the backbone. The $1595 \mathrm{~cm}^{-1}$ peak is due to some aromatic derivative, likely the quadrant stretch of some benzene derivative used in the clear coat. ${ }^{59}$ Styrene was ruled out as it exhibits a strong, characteristic peak at $1000 \mathrm{~cm}^{-1}$, which man- 
ifests at a positive factor loading in PC 1 . The $1732 \mathrm{~cm}^{-1}$ peak is the characteristic carbonyl stretch, attributed to the acrylic binder of most of the clear coats examined.

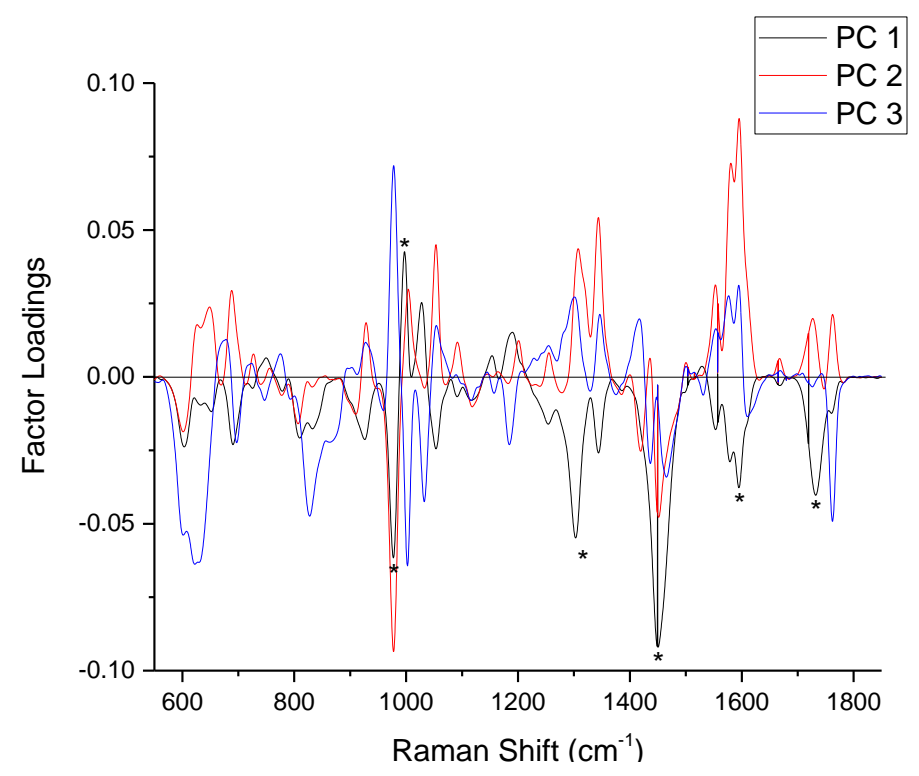

Figure 3.16 Factor loadings of the first three PCs for clear coat Raman spectra taken with the $785 \mathrm{~nm}$ laser. Asterisks identify the highest intensity peaks in the factor loadings of each PC.

Example Raman spectra of the different clear coat chemistries identified in the sample set are shown in Figure 3.17. Annotation colors correspond to the chemistries as follows: acrylic $=$ blue, urethane $=$ red, melamine $=$ pink, styrene $=$ green. The clear coat spectrum of sample 58 (bottom) collected with the $785 \mathrm{~nm}$ laser exhibited prominent peaks corresponding to the melamine-modified acrylic binder. ${ }^{59}$ The peak at roughly $978 \mathrm{~cm}^{-1}$ is ascribed to melamine triazine ring breathing. The clear coat spectrum of sample 3 (middle) exhibited the characteristic acrylic peaks in the $1300 \mathrm{~cm}^{-1}$ to $1800 \mathrm{~cm}^{-1}$ region, and styrene peaks at lower 
frequencies. The peaks at $1304 \mathrm{~cm}^{-1}, 1448 \mathrm{~cm}^{-1}$, and $1728 \mathrm{~cm}^{-1}$ correspond to $\mathrm{CH}_{2}$ in-phase twisting vibrations, $\mathrm{CH}_{3}$ and $\mathrm{CH}_{2}$ deformations, and carbonyl stretches in the acrylic binder. The peaks at $620 \mathrm{~cm}^{-1}, 1000 \mathrm{~cm}^{-1}, 1030 \mathrm{~cm}^{-1}$, and $1190 \mathrm{~cm}^{-1}$ correspond to ring deformation, trigonal ring breathing vibrations, and the $\mathrm{C}_{6} \mathrm{H}_{5}-\mathrm{C}$ stretching vibration due to the styrene modification..$^{45,59}$ The main peaks for melamine and styrene modification occur in roughly the same Raman shift range, increasing the potential for mistakes if Raman spectroscopy is solely used for spectroscopic analysis. This highlights the importance of utilizing multiple methods in clear coat analysis. In such cases, the most specific peaks should be used for determining presence of these modifications: the $1000 \mathrm{~cm}^{-1} / 1030 \mathrm{~cm}^{-1}$ peak pair for styrene and the $978 \mathrm{~cm}^{-1}$ peak for melamine. The clear coat Raman spectrum of sample 19A (top) was representative of the new generation "oxithane"-type acrylic-urethane binder previously discussed. The primary peak in the Raman spectra differentiating this type of clear coat from a typical acrylic styrene coating occurs at roughly $1760 \mathrm{~cm}^{-1}$, alongside the usual $1730 \mathrm{~cm}^{-1}$ carbonyl acrylic peak. This peak is due to the carbamate carbonyl structure of urethane and would confirm the coating is urethane-based in the absence of IR spectra, though not necessarily "oxithane"-type ${ }^{61,62}$ 


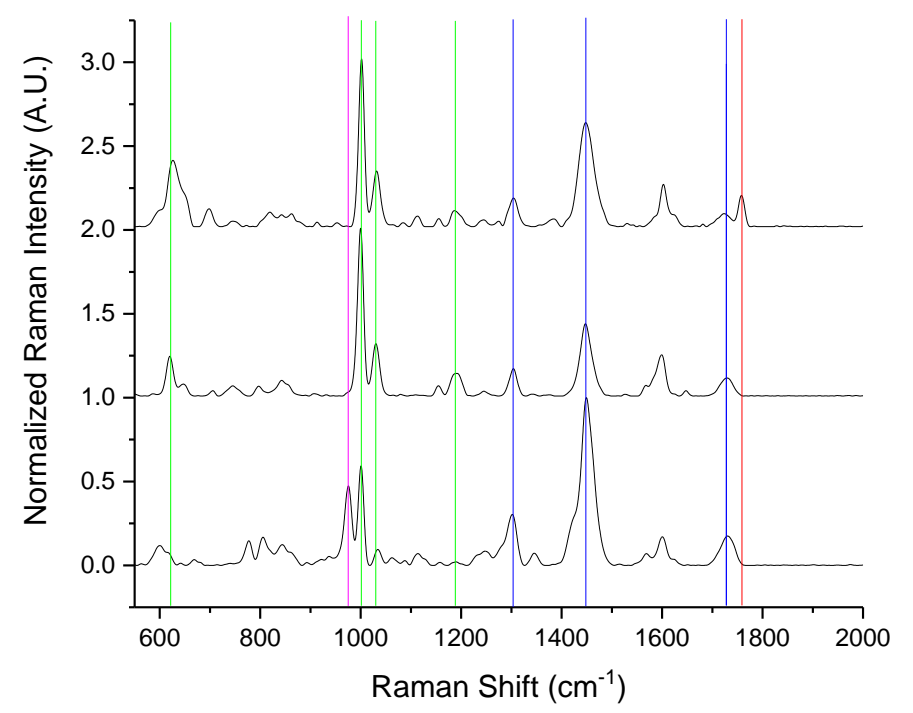

Figure 3.17 Raman spectra of (top) an oxithane acrylic urethane clear coat, (middle) an acrylic styrene clear coat, and (bottom) an acrylic melamine clear coat taken with the $785 \mathrm{~nm}$ laser. Vertical lines identify chemical composition analyzed in the discussion.

The $\mathrm{Cl}$ pigments in the base coats were identified by comparison to reference spectra of pure pigments available in the literature (Table 3.5). ${ }^{58}$ Most literature on Raman spectroscopy of automotive coatings utilize near-IR laser excitation, so the reference spectra were all collected with a $785 \mathrm{~nm}$ laser. Nevertheless, the peak patterns act as fingerprints of the pigments, which can be identified in sample spectra collected with different excitation sources regardless of differences in peak position. The Raman spectrum of the refinish base coat of sample 12 collected with the $785 \mathrm{~nm}$ laser indicated the layer contains PR254(a), an organic red pigment dikeopyrrolo-pyrrole (DPP); the region highlighted in the reference spectra correspond to the range of the sample spectrum shown (Figure A.1). ${ }^{58}$ This pig- 
ment was also found in the original base coat of the same sample, and in the original and refinish base coats of sample 13. The spectrum of the original base coat of sample 18A (Figure A.2) indicated the layer contains PB60, an organic blue pigment indathrone. ${ }^{58}$ This pigment was also found in the base coats of samples 19A, 34PS and 34PW. These results were expected as samples 34PS and 34PW were standards obtained from the manufacturer of the vehicles from which samples $18 \mathrm{~A}$ and $19 \mathrm{~A}$ were obtained. This pigment was also found in the spectra of the effect layers of sample $1 \mathrm{~A}$ collected with the $532 \mathrm{~nm}$ laser, demonstrating how pigments in base coats can be identified even if the reference and sample spectra are collected with different lasers. For all of the white samples, only rutile titanium dioxide (PW6) was observed in the base coat Raman spectra (Figure A.3), which corresponds with the IR spectroscopy results. Low power was used to avoid degradation of the highly absorbent black base coats, resulting in noisy spectra; however, carbon black in various forms was still distinguishable. Carbon black exhibits peaks at roughly $1300 \mathrm{~cm}^{-1}$ and $1600 \mathrm{~cm}^{-1}$, and the spectrum of the base coat of sample 10P taken with the $785 \mathrm{~nm}$ laser exhibited peaks characteristic to the PBK7 form of carbon black (Figure A.4).

Finally, the initial rationale for collecting spectra with both excitation sources was the concept that certain pigments in a sample may exhibit preferential Raman scattering towards a source. This was observed in the base coat of sample 19A, which exhibited spectra containing peaks corresponding to the organic violet pigment dioxazine (PV23( $\beta)$ ) when excited with the $532 \mathrm{~nm}$ laser, and peaks corresponding to the organic blue pigments phthalocyanine (PB15) and PB60 with the 
$785 \mathrm{~nm}$ laser (Figure A.5); the red arrows indicate the PB60 peaks and the blue arrow indicates the peak corresponding to PB15. ${ }^{58}$ This phenomenon has been observed in the literature on Raman spectroscopy of blue automotive paints. ${ }^{43}$

\subsection{Conclusions}

The sample set of automotive coatings analyzed represented vehicles manufactured no earlier than 2000, the five most common colors (black, grey, white, blue, and red), and effect and flat paints on metal and plastic substrates. Though the number of samples was insufficient to identify groups based on manufacturer or plant by PCA, the results support the development of standards for more facile and consistent spectroscopic analysis of automotive coatings in forensic laboratories. By preparing samples with microtomy and using transmission FTIR spectroscopy, cross sections of entire fragments can be analyzed at once. Similarly, for Raman microspectroscopy, embedding the sample and analyzing the cross section allows all layers to be analyzed with one sample preparation. For transmission IR spectroscopy, sections should be microtomed to ensure the sufficient transmission, and the spectral range widened down to roughly $500 \mathrm{~cm}^{-1}$ to ensure all additive peaks are detected. IR spectral analyses should focus primarily on binder identification in all layers, and additive identification in under coats.

For Raman spectroscopy, samples should be embedded in a resin so the cross section of the fragment with all the layers is visible. Microtoming cross sections is not recommended as the depth of penetration of the excitation sources employed in non-confocal Raman instruments can result in substrate interference. All clear coat spectra exhibited low signal to noise ratio and substrate interference 
when Raman microspectroscopy on microtomed cross sections was attempted. By analyzing the fragment cross section along the entire length of the sample, signal from the clear coat is amplified. In the literature, Raman spectroscopy of automotive clear coats has either been performed on compressed thin peels via macroscopic spectroscopy or by suspending the cross section on a sample holder with tape, avoiding substrates altogether. ${ }^{45,59}$ The former method is impractical as only fragments of clear coats may be acquired in forensics cases while the latter requires greater care in sample preparation, increasing overall analysis time. Finally, near IR excitation is recommended for analysis of clear coats to avoid inducing fluorescence and to improve signal to noise in spectra. However, base coats should be analyzed with all available lasers as pigments may exhibit preferential scattering with different excitation sources. If fluorescence overwhelms spectra, photobleaching for at least one minute and at a power greater than the sampling power is recommended to minimize analysis time and obtain some peaks. Comparison of peak pattern to reference spectra available in the literature should be used to identify pigments, bearing in mind shifts in peak location may occur if different excitation sources were used to collect the spectra. Ideally, known samples should be embedded alongside questioned fragments and analyzed in tandem to minimize errors associated with sample preparation. The binder and additive information obtained by IR spectroscopy combined with the pigment information derived from Raman spectroscopy should enhance efficacy of known-questioned analyses of automotive coating fragments in forensic cases. 


\section{Accelerated Weathering of Automotive Clear Coats}

\subsection{Introduction}

A final critical consideration in the characterization of unknown automotive coating fragments is sample age and history. This may be approximated by gauging the level of environmental damage incurred by the clear coat, as this layer incurs the majority of physical and chemical damage during service. The broad term "weathering" refers to all environmental factors that degrade the integrity of the clear coat and its ability to protect the remainder of the coating, including UV radiation, humidity or moisture, and temperature. For automotive coatings, UV radiation is typically the most damaging aspect of exposure. Light-induced damage is generally referred to as photodegradation. For exposed polymeric materials, photolysis and photo-oxidation describe two mechanisms of UV degradation. ${ }^{63}$ Photolysis is the homolytic cleavage of bonds in the absence of oxygen whereby absorption of radiation directly causes chain scission or radical formation in the backbone; this is characterized by Norrish Type I reactions commonly occurring in polyesters with aromatic backbone structures. ${ }^{64,65}$ Photo-oxidation is defined by radiation absorption initiating the formation of radicals that react with oxygen to produce reactive peroxy radicals, which propagate to further degrade the coating matrix; like photolysis, this can also result in chain scission. ${ }^{65}$ In this context, photooxidation will always occur and its mechanisms dominate sample degradation because automotive coatings are exposed in aerobic conditions.

In the presence of water, exposure can degrade the structure of clear coats through hydrolysis. Moisture from humidity and rain contribute in two primary ways 
to coating degradation. First, water acts as a plasticizer to decrease the glass transition temperature of coatings which increases free volume, segmental motion of chains, and allows additional water to penetrate the matrix. Second, in environments with constantly changing humidity, the absorption and evaporation of moisture from the surface leads to the development of internal stresses and mechanical deformation, facilitating further moisture absorption. ${ }^{65}$ Hydrolysis can also induce the creation of UV chromophores that accelerate radiation absorption and photodegradation reactions. ${ }^{66,67}$ In one study, rate of photo-oxidation increased in acrylic melamine coatings exposed to increasing humidity when light intensity and temperature were kept constant. ${ }^{68}$ Precipitation can remove degradation products as they are formed, further exposing the bulk coating. Finally, UV absorption also tends to raise the surface temperature of coatings, accelerating moisture absorption. Moisture is the most difficult exposure factor to quantify and control in accelerated studies due to variations in experimental setup. Studies utilizing physical water sprays and controlled or variable relative humidity can be found ${ }^{65}$ However, models of the effect of relative humidity on photo-oxidation rate have been developed from accelerated exposure experiments (Figure 4.1).69 


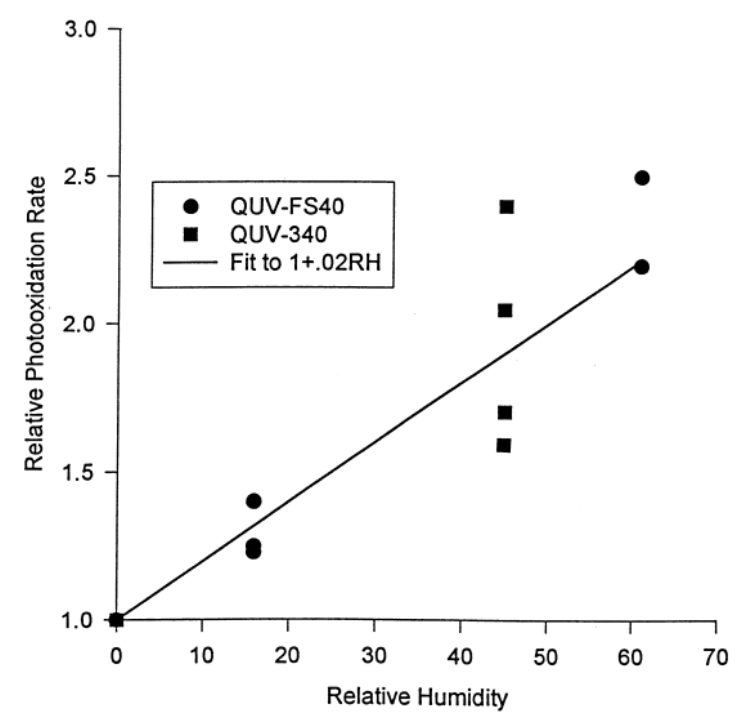

Figure 4.1 Effect of relative humidity on photo-oxidation rate of acrylic-melamine coatings. ${ }^{69}$

Temperature and UV radiation are not independent variables in coatings degradation because absorption of radiation increases the surface temperature of coatings. Differences in surface versus ambient temperature complicate outdoor weathering studies and UV radiation must be measured simultaneously. Temperature influences degradation primarily by providing sufficient energy to activate and accelerate reactions. Total damage (D) incurred over time $(\mathrm{t})$ is related to the light intensity (I), activation energy $\left(E_{a}\right)$, and temperature $(T)$ based on Equation 3, where $\mathrm{R}$ is the gas constant and $\mathrm{A}$ is the Arrhenius pre-exponential factor combining the proportionality constants for pure irradiance. ${ }^{65}$ Units for damage are based on intensity and the Arrhenius factor, which is based on the reaction order. Based on the literature, activation energy for photo-oxidation of polymers used in automotive coatings is roughly $6.5 \mathrm{kcal} / \mathrm{mol} .{ }^{70}$ The effect of temperature on reaction rate has also been studied using a modified form of the Arrhenius equation where 
$\mathrm{K}$ is the reaction rate (Equation 4). ${ }^{65}$ Based on an activation energy of $5 \mathrm{kcal} / \mathrm{mol}$, reaction rate increases $33 \%$ for every $10^{\circ} \mathrm{C}$ increase above ambient temperature. ${ }^{65}$ These are important considerations because the color of the base coat can also affect how strongly the whole coating absorbs radiation, resulting in surface temperatures of $38^{\circ} \mathrm{C}$ to $66^{\circ} \mathrm{C} .^{70}$

$D=\sum_{t} I_{t} A \exp \left(-\frac{E_{a}}{R T_{t}}\right) \Delta t$

$\ln K=-\left(\frac{E_{a}}{1.987}\right)\left(\frac{1}{T}\right)+A$

UV radiation dominates degradation of exposed coatings, so quantifying absorbed radiation is an important focus of many weathering studies. Quantum efficiency is often used to describe the ratio of photons absorbed by a sample to incident photons. Models and controlled studies of apparent or relative quantum efficiency of automotive coatings with radiation intensity and wavelength range have been conducted. ${ }^{63,65}$ One study illustrated the effect of wavelength range on quantum efficiency of acrylic and ester automotive coatings (Figure 4.2). ${ }^{70}$ The quantum efficiency of ester coatings increases significantly below roughly $300 \mathrm{~nm}$. The little radiation observed below $300 \mathrm{~nm}$ in outdoor exposure during the summer is not a significant issue in terms of different material sensitivities to wavelength range. ${ }^{70}$ Therefore, wavelength range should not be a significant issue for accelerated weathering of different automotive coatings if the artificial source is similar to sunlight. Degradation mechanism does not appear to be distorted when radiation is increased in weathering studies; however, surface temperature must remain well controlled to prevent altering photodegradation mechanisms. ${ }^{70}$ Accelerated 
weathering studies must apply aggressive conditions without altering the mechanisms that occur during outdoor exposure.

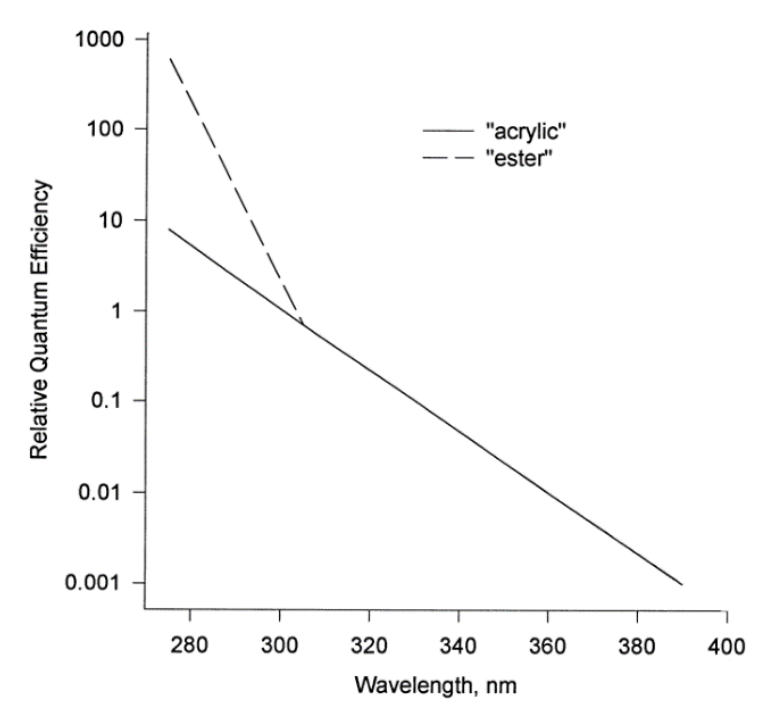

Figure 4.2 Action spectrum illustrating the effect of wavelength range on quantum efficiency. ${ }^{70}$

Since photolysis primarily occurs in polyesters under anaerobic conditions, photo-oxidative mechanisms are the primary concern. However, photolysis plays an important role in initiation of photo-oxidative reactions by reacting with functional groups containing $\pi$-electrons such as carbonyls, carbon-carbon double bonds, and aromatic groups. In acrylic coatings, these are typically carbon-carbon double bonds and aromatic impurities from solvents or other reagents remaining after polymerization. ${ }^{65}$ Photodegradation progresses as a series of radical reactions in three stages, akin to radical polymerization: initiation, propagation and/or transfer, and termination. Initiation occurs when UV radiation is absorbed by the polymer, producing highly reactive radicals. These radicals propagate and react with other species to transfer the radical, or with oxygen to form peroxides that cleave into peroxy radicals. Alkoxy and alkyl radicals also form as intermediates, 
which can lead to chain branching when alkyl radicals form new crosslinks or chain scission occurs. ${ }^{65}$ The series of reactions terminates when two radicals recombine, and this produces different functional groups that can be used to quantify degree of photo-oxidation. This process for a polyester results in shorter, hydroxyl-terminated chains (Figure 4.3)..$^{71}$ These reactions can also result in carboxylation and anhydride formation in polyesters. ${ }^{72}$

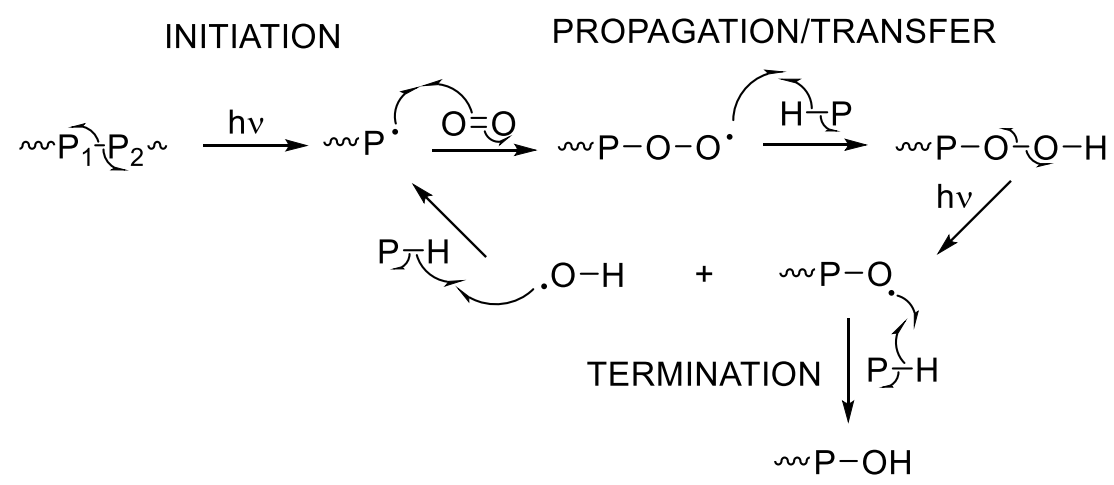

Figure 4.3 General scheme of polyester photo-oxidation. ${ }^{71}$

A final consideration in the photodegradation mechanism is the susceptibility of crosslinks to degradation. The mechanisms and products of melamine and urethane crosslinked acrylic coating degradation have been well characterized. The reactive sites in both of these structures are the $\alpha$-methylene carbon-hydrogen bonds. ${ }^{71,72}$ In melamine-crosslinked polyesters, photo-oxidation reactions can produce formate, amine, and formamide functionalities. In urethane-crosslinked polymers, acetylurethane functionalization can form while the carbamate functionality is retained (Figure 4.4).$^{72}$ By understanding the mechanisms of photodegradation, accelerated weathering of automotive coatings can be studied by quantifying the functional groups formed or degraded. This is important for service life prediction 
of coatings for manufacturers, but could also provide forensic examiners with information on the age and history of unknown samples. 
(a)

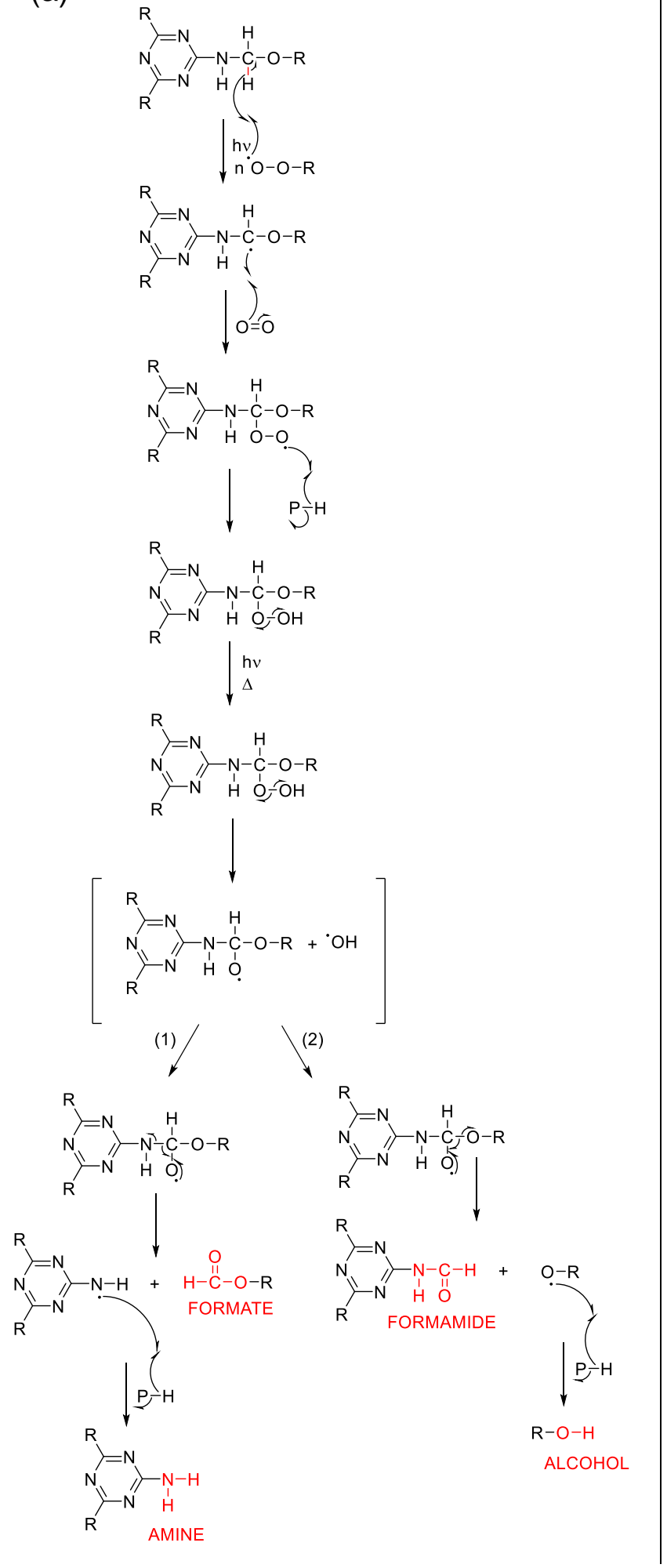

(b)

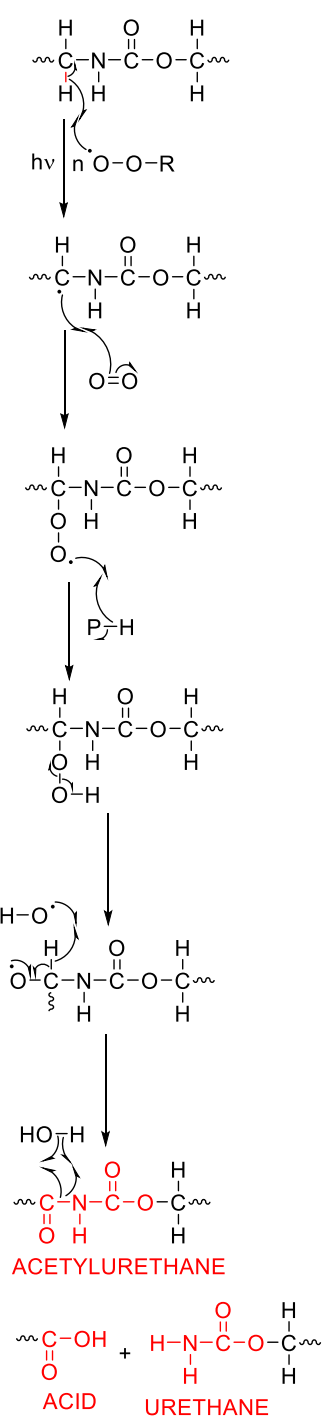

Figure 4.4 Melamine (a) and carbamate (b) crosslink photodegradation reactions. ${ }^{72}$ 
ATR FTIR spectroscopy can be used to track photodegradation. The intensity of bands due to vibrations of major functional groups change as the matrix degrades. This method has been used in the literature to study weathering of a number of polymeric systems including epoxy, acrylic urethane, and polyester urethane. ${ }^{73-75}$ The method involves determining which peaks significantly decrease or increase in intensity during weathering, and tracking the ratio of their intensity during weathering to their initial intensity after normalization to a more stable peak. In thermally-induced photodegradation, chain scission can occur in which the backbone breaks to yield free radicals or a small molecule is eliminated and the backbone reforms a double bond. ${ }^{60}$ These reactions disrupt the methylene backbone structure in acrylates, resulting in a decrease of the peak in the $2900 \mathrm{~cm}^{-1}$ region. A second common peak of interest is the carbonyl peak in the $1720 \mathrm{~cm}^{-1}$ to $1730 \mathrm{~cm}^{-1}$ region. This heterogeneous bond is typically part of an ester group susceptible to photodegradation reactions. The broad band in the $3000 \mathrm{~cm}^{-1}$ region corresponding to hydroxyl and amine vibrations is tracked because the degradation of melamine and polyester structures results in termination of chains with these functional groups. ${ }^{72}$ Since Raman spectroscopy has proven successful for characterizing the binder chemistry of automotive clear coats, it will be used here as well to determine whether peaks corresponding to major binder structures change similarly with weathering.

To prevent and retard the effects of photo-oxidation, manufacturers incorporate UV absorbers (UVAs) and hindered amine light stabilizers (HALS) into clear coats. ${ }^{46}$ UVAs tend to have aromatic structures that absorb and dissipate radiation 
as thermal energy, protecting the binder. Common UVAs include hydroxyphenylbenzotriazole, benzophenone, oxanailide, and triphenyltriazine (Figure 4.5 top). ${ }^{31,76}$ These structures represent the major classes of UVAs currently employed. HALS are derivatives of 2,2,6,6-tetramethyl piperidine and act as radical scavengers to prevent propagation of species formed by photooxidation reactions from further damaging the binder (Figure 4.5 bottom). ${ }^{65} \mathrm{HALS}$ form stable nitroxide radicals from the reaction of hydroxylamine with peroxy radicals resulting in regeneration, thereby providing good, long-term stabilization in small concentrations. ${ }^{65}$ However, the reactive nature of radicals makes this process difficult to observe in accelerated aging studies by facile analytical techniques. Since UVAs absorb radiation, their presence and concentration are easily measured by spectrophotometric methods. Therefore, most exposure studies quantifying stabilizer concentration measure UVA concentration as an estimate of the extent of degradation. ${ }^{77}$

(a)

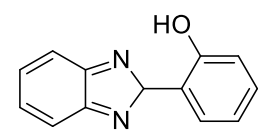

hydroxyphenylbenzotriazole (b)

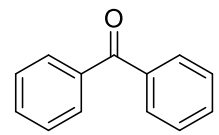

benzophenone

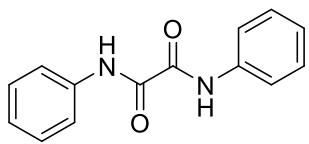

oxanilide

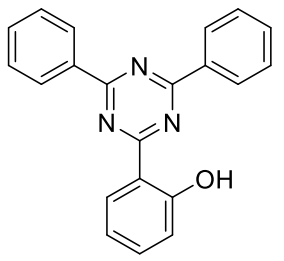

triphenyltriazine

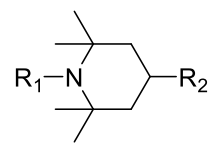

2,2,6,6-tetramethyl piperidine: $\mathrm{R}_{1}=\mathrm{H}, \mathrm{R}_{2}=\mathrm{H}$

Figure 4.5 Structures of common UVAs (a) and HALS (b) in clear coats.

To characterize the effects of exposure on clear coats, quantification of UVA concentration by T-MSP has been examined. Preparation for adequate transmission is simpler as the layer is transparent. ${ }^{76}$ Liszewski et al. successfully identified 
the UV absorbing compounds in clear coats using T-MSP; however, their chemometric analysis showed no correlation between the combination and quantities of UVAs in a clear coat, and identifying properties of the vehicle such as make, model, and year. ${ }^{76}$ This suggests spectrophotometric analysis of clear coats for forensic purposes may only be suitable for known-questioned comparisons for age and history. The concentration of UVAs in clear coats also vary with depth because the surface is exposed to more radiation over time. T-MSP has proven a suitable method for UVA identification and quantification with increasing exposure. ${ }^{78}$

Weathering also results in visible physical changes to the appearance of clear coats. The most significant and commonly studied properties in the literature are yellowing and gloss decrease. ${ }^{78,79}$ These are based on measurements using commercial color and gloss meters. Yellowing index $(\mathrm{YI})$ is the most commonly reported metric for yellowing, and is defined by ASTM color standards according to spectrophotometric data using the tristimulus values $(\mathrm{X}, \mathrm{Y}$, and $\mathrm{Z})$ and constants ( $C_{x}$ and $C_{z}$ ) based on the standard illuminant and observer functions used (Equation 5). ${ }^{80}$ Yellowing of polyester coatings is reported in the literature, and postulated to be the result of aromatic crosslinking reactions during photodegradation forming a conjugated system of chromophores. ${ }^{66}$ The presence of water in high humidity or moisture environments promotes increased roughness of the coating surfaces. Hydrolytic reactions during photodegradation occur at the hydrophilic regions of the heterogeneous matrix; hydrolysis at these regions results in pits contributing to overall roughness and decreased gloss in weathered coatings. ${ }^{66}$ The heterogeneity of acrylic melamine coatings is the result of low molecular weight, partially 
polymerized gel-like regions remaining within the crosslinked network after curing. ${ }^{81}$

$Y I=\frac{100\left(C_{x} X-C_{Z} Z\right)}{Y}$

A final consideration in weathering studies is that automotive manufacturers typically apply clear coats onto the wet base coat prior to curing the entire system. Since migration of UVAs can occur with this type of application, weathering studies of automotive clear coats should be performed on full coating systems. This section will address the aging of clear coats by employing accelerated artificial weathering on pristine samples received from two of the three coatings manufacturers holding over 90 percent of the North American automotive coatings market. ${ }^{31}$ Chemical and physical degradation will be tracked with spectroscopy and appearance measurements, respectively.

\subsection{Experimental}

Cured clear coats were obtained as part of full paint systems (clear coat, base coat, primer surfacer, and primer) on steel ACT panels directly from the manufacturers (Table 4.1). All the samples had flat, non-metallic white and black base coats except those noted with asterisks. Free films of two samples were also studied. "Type" refers to the designation on the manufacturer's label upon receipt, while "Class" was either provided on the sample label or determined from datasheets. ${ }^{82}$ 
Table 4.1 Properties of Clear Coats Studied

\begin{tabular}{ccccc} 
Sample & Manufacturer & Type & Base Coat Color & Class \\
\hline BEB & BASF & E10CG081G & black & $2 \mathrm{~K} 4$ \\
BEF & BASF & E10CG081G & free film & $2 \mathrm{~K} 4$ \\
BEW & BASF & E10CG081G & white & $2 \mathrm{~K} 4$ \\
BRB & BASF & R10CG392D & black $^{*}$ & $1 \mathrm{~K}$ high solids \\
BRF & BASF & R10CG392D & free film & 1K high solids \\
BRW & BASF & R10CG392D & white & $1 \mathrm{~K}$ high solids \\
PAB & PPG & APO & black & $2 \mathrm{~K}$ \\
PAW & PPG & APO & white & $2 \mathrm{~K}$ \\
PNB & PPG & NCT & black & $2 \mathrm{~K}$ \\
PNW & PPG & NCT & white & $2 \mathrm{~K}$ \\
PTB & PPG & TMAC & black* & \\
PTW & PPG & TMAC & white & \\
* base coat contains metallic flakes & & &
\end{tabular}

Artificial weathering at $55^{\circ} \mathrm{C} \pm 3.5^{\circ} \mathrm{C}, 75 \%$ relative humidity $(\mathrm{RH}) \pm 5 \%$, and with $100 \%$ UV radiation was carried out in the NIST SPHERE. ${ }^{83}$ Metal sample holders with 19-mm diameter circular cut outs in positions of known irradiance were used so cumulative dose for each sample could be calculated. For each fullsystem sample, two $20-\mathrm{mm}$ by $20-\mathrm{mm}$ squares were prepared with a sheet metal shearer. The first sample was maintained for non-destructive color and gloss measurements roughly every three days, while the second was analyzed by ATR FTIR spectroscopy every five days. A third sample was prepared for Raman and MSP analyses in the following manner: strips of the paint panels were cut and bent with pliers to release the system from the substrate, and 4-mm diameter samples were manually punched out. A 19-mm steel disk was covered with double-sided tape and the samples arranged as close as possible on the tape; each disk fit a minimum of 10 full samples (4-mm circles). The disk was flipped upside down on silicone paper and pressed to ensure the samples would remain adhered during 
exposure. A fragment of roughly half a 4-mm sample was removed once a week for Raman and MSP sample preparation.

Free films of some clear coats were additionally weathered to examine the effect of the base coat on photodegradation. These samples were provided as drawdowns on release paper by the manufacturer. Samples were prepared from areas labeled as $60 \mu \mathrm{m}$ thick for ease of handling. One $20-\mathrm{mm}$ by $20-\mathrm{mm}$ sample was cut from the release paper and the film carefully removed with tweezers for ATR FTIR spectroscopy measurements. A second sample larger than 19-mm was removed and taped onto the SPHERE sample holder on top of a steel disk, so samples could be removed for MSP and Raman spectroscopy analyses. As these samples had no substrate, color and gloss measurements were not performed.

Color measurements were obtained with a BYK spectro-guide sphere gloss color meter employing $\mathrm{d} / 8$ geometry with the specular component included, using the D65 illuminant and $10^{\circ}$ standard observer functions. Samples were cleaned with lint-free wipes prior to measurement, and placed in a sample holder with 11-mm diameter sampling area. Four measurements at the same location of each sample at each time point were taken to obtain CIELAB color coordinates and $\mathrm{YI}$, calculated according to ASTM E313-98 by the instrument. ${ }^{29,80}$ The CIELAB coordinates and $\mathrm{YI}$ were averaged for each sample at each time. Change in YI was tracked for the white samples, and change in $\mathrm{b}^{*}$ and $\mathrm{DE}^{*}$ were tracked for all samples. Gloss measurements were taken at $20^{\circ}$ and $60^{\circ}$ geometries with a BYK micro-TRI-gloss meter. The measurement areas reported by the instrument manu- 
facturer for these geometries are $10-\mathrm{mm}$ by $10-\mathrm{mm}$ and $9-\mathrm{mm}$ by $15-\mathrm{mm}$, respectively, so the sample size was sufficient to prevent edge irregularities from affecting measurements. ${ }^{84}$ Four measurements of each sample were taken, averaged, and gloss retention tracked with dose. Standard deviations were calculated for the four measurements at each time point. Error in $\mathrm{YI}$ and $\mathrm{b}^{*}$ change, and $\mathrm{DE}^{*}$ were calculated using the simplified propagation of error formula, where $s$ is the standard deviation for the given variable and $f$ is a function of $x, y$, and $z$ (Equation 6). This is a method for describing error of a function calculated using variables with defined errors when lacking multiple measurements of the function.

$s_{f}=\sqrt{\left(\frac{\partial_{f}}{\partial_{x}}\right)^{2} s_{x}^{2}+\left(\frac{\partial_{f}}{\partial_{y}}\right)^{2} s_{y}^{2}+\left(\frac{\partial_{f}}{\partial_{z}}\right)^{2} s_{z}^{2}+\cdots}$

Macroscopic and microscopic ATR FTIR spectra were obtained using Nicolet iS50 and iN10MX spectrometers employing room-temperature DTGS and liquid nitrogen-cooled MCT-A detectors, respectively. Spectra were collected from (300 to 4000$) \mathrm{cm}^{-1}$ and from ( 675 to 4000$) \mathrm{cm}^{-1}$, respectively. For both instruments, spectra of $4 \mathrm{~cm}^{-1}$ resolution and 128 scans averaged were collected. Pressure on the iN10 MX instrument was set at 40 psi for the full system samples and $15 \mathrm{psi}$ for the free films to prevent the sample from adhering to the crystal between sampling locations. The ATR crystals of the iS50 and iN10 MX instruments were made of diamond and germanium yielding approximate penetration depths of 0.65 and $1.66 \mu \mathrm{m}$, respectively. ${ }^{85}$ As clear coats are typically no less than $10 \mu \mathrm{m}$ thick, interference from the base coats should not occur, and was confirmed by comparing the spectra of the clear coats on white and black taken with both instruments prior 
to the study. At each sampling time, five spectra were collected at random locations on the sample. Background spectra were obtained prior to each new sample, and the crystals were cleaned with methanol and lint-free wipes prior to each background spectrum. Spectra were processed with OriginLab software. The same baseline was manually subtracted from each replicate spectrum, and the same baseline used for samples of the same chemistry regardless of base coat color.

The weathered FTIR spectra were subtracted from the initial spectrum to determine which peaks would be suitable for tracking mass loss and oxidation. Since no peaks remained unchanged in the samples examined, ratios of peaks in similar regions were calculated to track these changes. For mass loss, the peak intensity at $2830 \mathrm{~cm}^{-1}$ was normalized to the $2930 \mathrm{~cm}^{-1}$ peak. For oxidation, the peak intensity at $1680 \mathrm{~cm}^{-1}$ was normalized to the $1455 \mathrm{~cm}^{-1}$ peak. Because the depth of penetration for ATR crystals changes with wavenumber, normalization peaks as close as possible to the peak of interest were chosen. The $2930 \mathrm{~cm}^{-1}$ and $1455 \mathrm{~cm}^{-1}$ peaks represent $\mathrm{sp}^{3} \mathrm{C}-\mathrm{H}$ stretching and bending, respectively, and were present in the spectra of all the samples, permitting comparison between the different chemistries studied. The ratio for each weathered spectrum, $A(t)$, was then divided by the ratio for the unweathered spectrum, $A(0)$. Linear regression was performed in OriginLab to gauge degradation rate differences between samples.

For MSP analysis, the removed fragment was sandwiched between reference films of known UVA concentration prior to microtoming cross sections. This procedure was adapted from the literature and is necessary to account for the difference between the measurement direction (perpendicular to the cross section) 
and direction of interest (parallel to the cross section) for quantification of UVA concentration. ${ }^{77}$ Acrylic urethane reference films with 2 percent UVA and 1.5 percent HALS by weight were prepared with 10 mil wet film thickness on Mylar. Benzotriazole-type UVAs were employed (Figure 4.6). The absorbance of this film at $345 \mathrm{~nm}$ was measured with a Lambda 900 spectrophotometer to obtain an absorbance per micron factor. The weathered samples were sandwiched between two pieces of this reference film with a polyurethane-based solvent-free adhesive (LORD Fusor 148), and cross sections of $5 \mu \mathrm{m}$ thickness were obtained by roomtemperature microtomy (Leica EM UC7) after embedding in epoxy (Section 3.2). Sections were mounted with glycerol on a quartz slide with a quartz coverslip, which was flattened with an Excel roller immediately prior to analysis. Spectra were collected in transmission mode with a CRAIC MP-2 UV-vis spectrophotometer with a 36X (NA 0.5) objective from $200 \mathrm{~nm}$ to $800 \mathrm{~nm}$ employing a square aperture of roughly $7.9 \mu \mathrm{m}, 20 \mathrm{~ms}$ integration time, and 25 averaged scans. Spectra were collected at three different locations. At each location, a spectrum was collected (1) near the surface of the clear coat, (2) in the center of the clear coat, and (3) closest to the clear coat-base coat interface. Lastly, spectra of the reference films were collected. Spectra were processed with OriginLab software. 
(a)

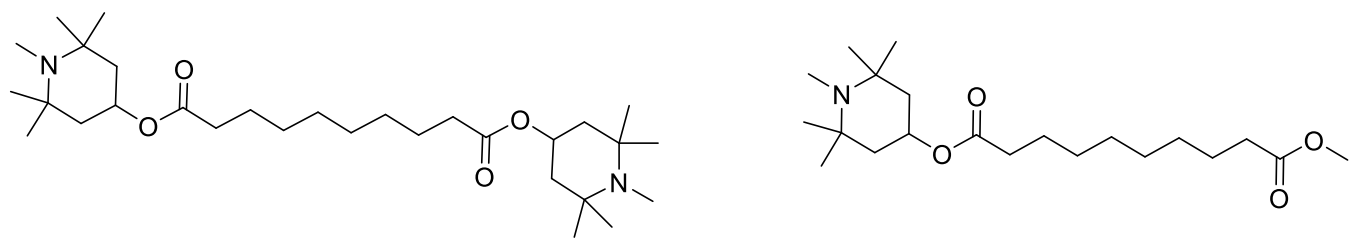

(b)
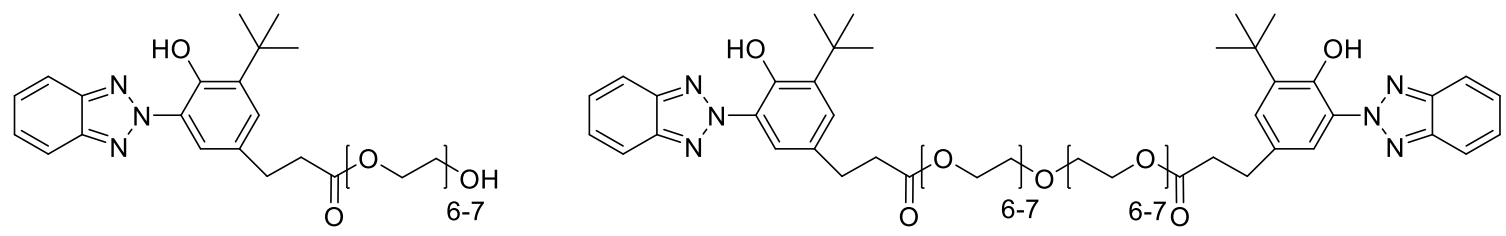

Figure 4.6 Structures of (a) HALS and (b) UVA used in reference film.

For each clear coat chemistry, the raw spectra were first examined to determine the type(s) of UVA present. A peak specific to each UVA was chosen as the absorbance used to calculate total clear coat absorbance (TCA), a quantity representing the concentration of UVAs. For sample thickness correction, each sample spectrum was first normalized using the absorbance per micron factor of the reference film, and absorbance of the reference film in the cross section. The absorbance of the UVA peak of interest in the sample spectrum was multiplied by the ratio of aperture size $(7.9 \mu \mathrm{m})$ to the corrected cross section thickness to obtain absorbance in the direction of interest (along the clear coat depth). The absorbance values from the three spectra collected at each location were summed to obtain TCA. TCA at the three locations was averaged and plotted at each time to track UVA degradation over the course of the weathering experiment.

For Raman microspectroscopy, the embedded MSP sample was microtomed for surface smoothness. Spectra were collected with a Bruker Senterra spectrometer and $785 \mathrm{~nm}$ excitation source using a 50X (NA 0.40) objective. Laser power and integration times used are listed in Table 4.2. As surface roughness can affect Raman intensity, spectra were collected every $10 \mu \mathrm{m}$ from the surface 
along the depth of the clear coat to normalize variations in sample preparation within each sample. Each spectrum was baseline corrected with Bruker OPUS software using the concave rubberband correction method with 20 iterations. Then, the ratio of the peak of interest to a nearby peak was calculated, and this ratio at $10 \mu \mathrm{m}$ from the surface divided by the ratio at $30 \mu \mathrm{m}$ from the surface. Finally, the ratio of the weathered to unweathered samples was calculated.

Table 4.2 Raman Spectroscopy Parameters for Clear Coats

\begin{tabular}{cccc} 
Sample & Laser Power $(\mathbf{m W})$ & Integration Time $(\mathbf{s})$ & Number of Co-additions \\
\hline BEB & 10 & 45 & 2 \\
BEF & 10 & 45 & 2 \\
BEW & 1 & 45 & 2 \\
BRB & 10 & 45 & 2 \\
BRF & 10 & 45 & 2 \\
BRW & 1 & 45 & 2 \\
PAB & 10 & 45 & 2 \\
PAW & 10 & 45 & 2 \\
PNB & 10 & 45 & 2 \\
PNW & 10 & 45 & 2 \\
PTB & 10 & 45 & 2 \\
PTW & 1 & 45 & 2
\end{tabular}

\subsection{Results and Discussion}

The raw ATR FTIR and Raman spectra of the unexposed BASF and PPG clear coats are shown in Figure 4.7 and Figure 4.8, respectively. These spectra were collected of the samples on white base coats except the Raman spectrum of the PPG TMAC clear coat, which was collected using a black sample due to overwhelming fluorescence in the white sample spectrum. 

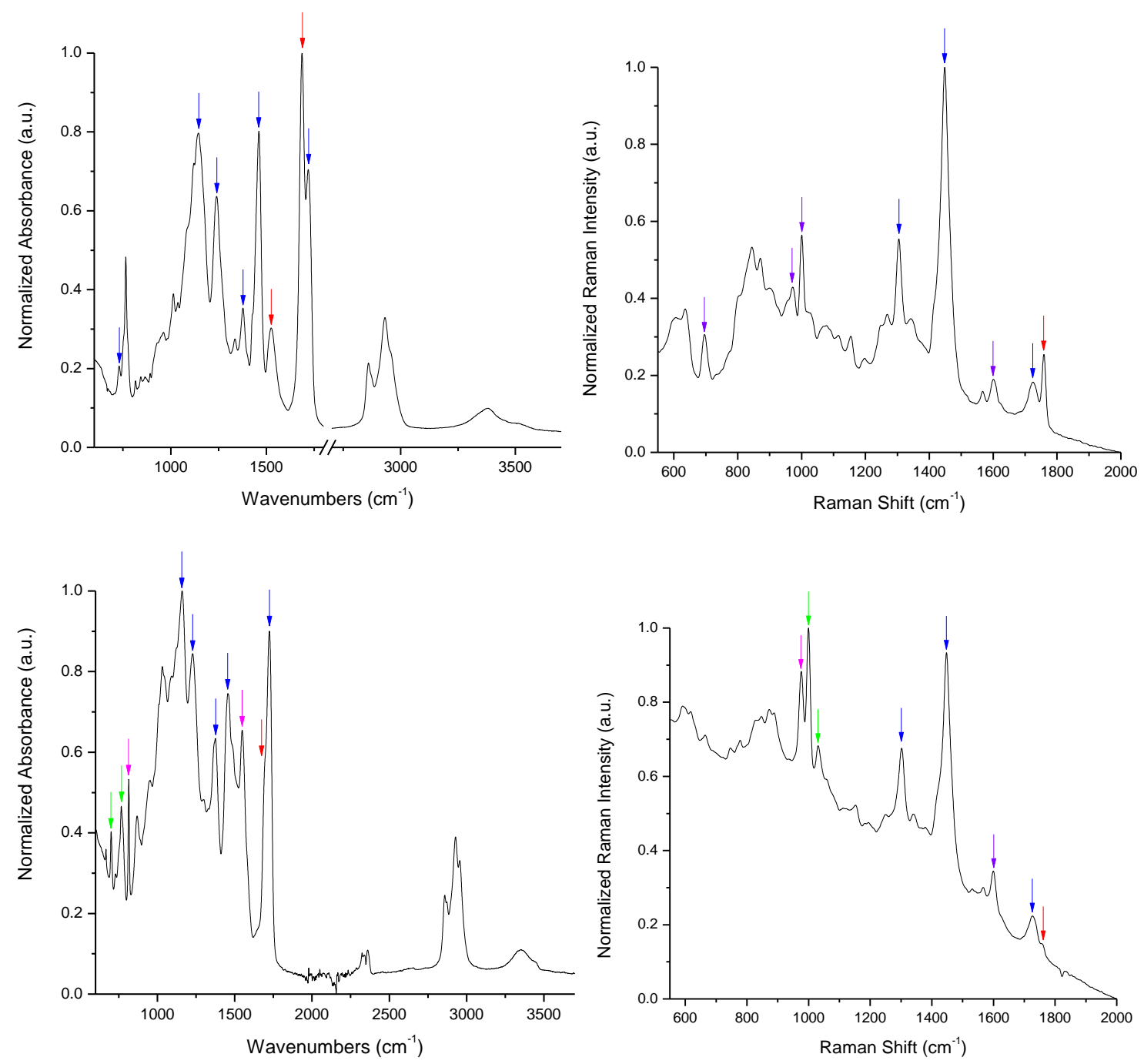

Figure 4.7 ATR FTIR (left) and Raman (right) spectra of BASF E10CG081G (top) and R10CG392D (bottom) clear coats. Blue, red, green, pink, and purple arrows indicate peaks ascribed to acrylic, urethane, styrene, melamine, and general aromatic structures, respectively.

The FTIR spectra of the BASF clear coats indicate the E10CG081G coating is acrylic urethane with some aromatic structures, and the R10CG392D coating is acrylic urethane with melamine and styrene. For the E10CG081G coating, this is based off the characteristic oxithane-type acrylic urethane stretches and the small 
$730 \mathrm{~cm}^{-1}$ peak appearing as a shoulder on the urethane $765 \mathrm{~cm}^{-1}$ peak. ${ }^{10}$ The $730 \mathrm{~cm}^{-1}$ peak is due to aromatic carbon-carbon double bond bending vibrations. For the R10CG392D coating, the urethane modification results in only a slight shoulder on the acrylic carbonyl peak. ${ }^{10}$ The peaks at $1550 \mathrm{~cm}^{-1}$ and $815 \mathrm{~cm}^{-1}$ are triazine vibrations indicating melamine modification, and the sharp peaks at $765 \mathrm{~cm}^{-1}$ and $700 \mathrm{~cm}^{-1}$ are indicative of styrene. These chemistries were confirmed by the manufacturer's datasheets.

The Raman spectra of the BASF clear coats confirms these binder assignments, with major peaks for acrylic, urethane, and aromatic structures present. Both coatings have sharp peaks at $1450 \mathrm{~cm}^{-1}$ and $1300 \mathrm{~cm}^{-1}$ due to methyl and methylene deformations corresponding to acrylic structures. A sharp peak at 1760 $\mathrm{cm}^{-1}$ corresponding to the urethane carbonyl vibration occurs in the E10CG081G spectrum. Urethane manifests as a shoulder on the $1725 \mathrm{~cm}^{-1}$ acrylic carbonyl peak in the R10CG392D spectrum. Intense peaks at $1030 \mathrm{~cm}^{-1}$ and $1000 \mathrm{~cm}^{-1}$ indicate styrene modification, and the sharp peak at $978 \mathrm{~cm}^{-1}$ is due to melamine. 

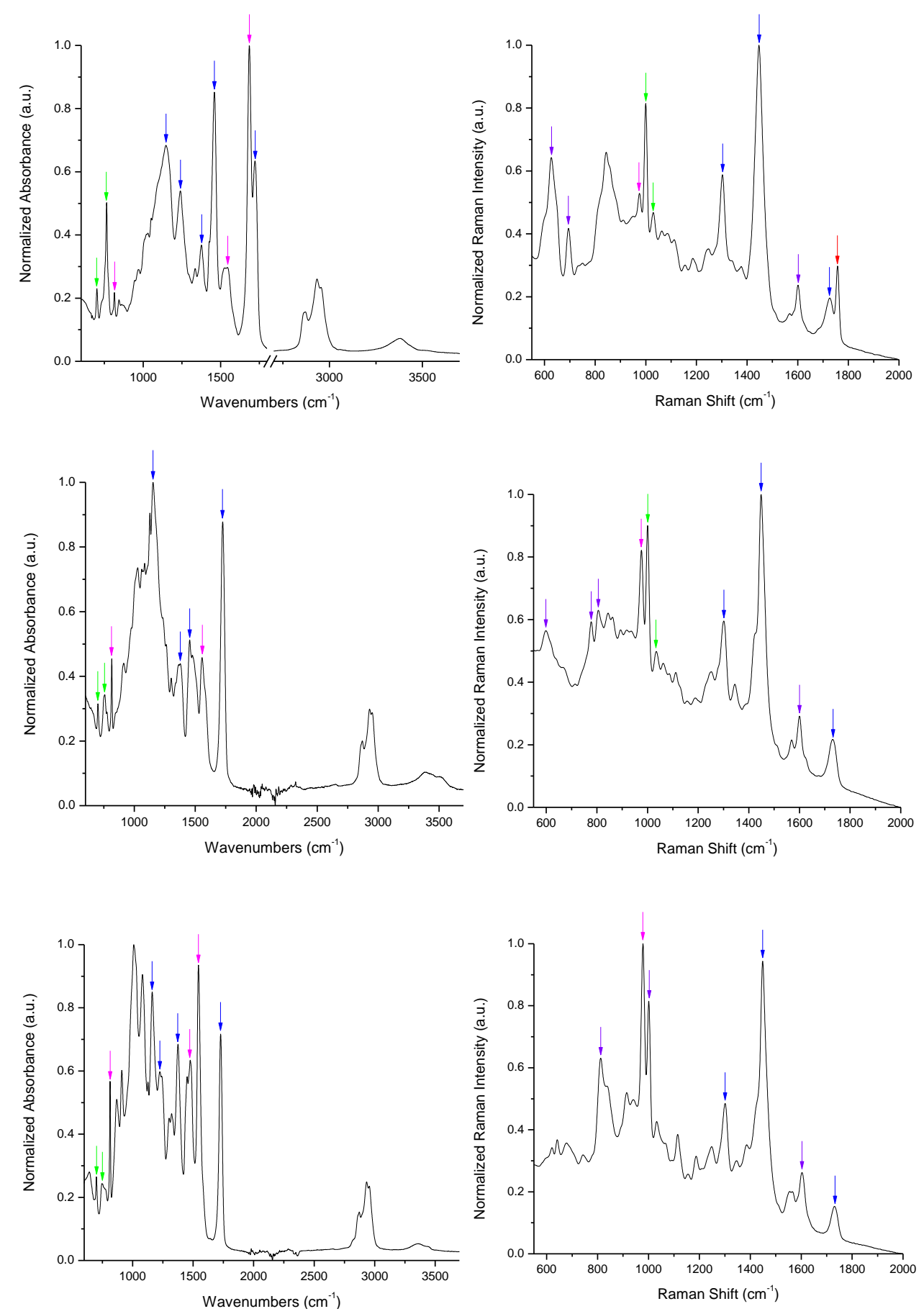

Figure 4.8 ATR FTIR (left) and Raman (right) spectra of PPG APO (top), NCT (middle), and TMAC (bottom) clear coats. Blue, red, green, pink, and purple arrows indicate peaks ascribed to acrylic, urethane, styrene, melamine, and general aromatic structures, respectively. 
Based on the FTIR spectra of the PPG clear coats, the APO coating is acrylic urethane melamine while the NCT and TMAC coatings are both acrylic melamine. All three have some aromatic structures, but the APO coating most likely contains styrene based on the intense $765 \mathrm{~cm}^{-1}$ and $700 \mathrm{~cm}^{-1}$ peaks. The Raman spectrum of the NCT coating indicates possible styrene modification based on the intense $1030 \mathrm{~cm}^{-1}$ and $1000 \mathrm{~cm}^{-1}$ peaks, though these are general phenyl deformation and trigonal ring breathing vibrations. All three coatings exhibit intense $978 \mathrm{~cm}^{-1}$ Raman peaks due to triazine trigonal ring breathing, confirming the presence of melamine.

The FTIR spectra of the clear coats with increasing dose are available in the Appendix (Figure A.6 and Figure A.7). To track mass loss and oxidation, the subtraction spectra of each coating were examined to determine the peaks to use for normalization within each spectrum (Figure 4.9). The peaks corresponding to symmetric methyl and asymmetric methylene acrylic vibrations at $2860 \mathrm{~cm}^{-1}$ and $2930 \mathrm{~cm}^{-1}$, respectively, were used to track mass loss. The $1672 \mathrm{~cm}^{-1}$ and 1460 $\mathrm{cm}^{-1}$ peaks corresponding to amide and methyl and methylene bending vibrations, 
respectively, were used to track oxidation. ${ }^{72}$ Degradation rates by chemical structure and base coat color were compared. Degradation rates from the macroscopic and microscopic ATR FTIR spectrometers were also compared.
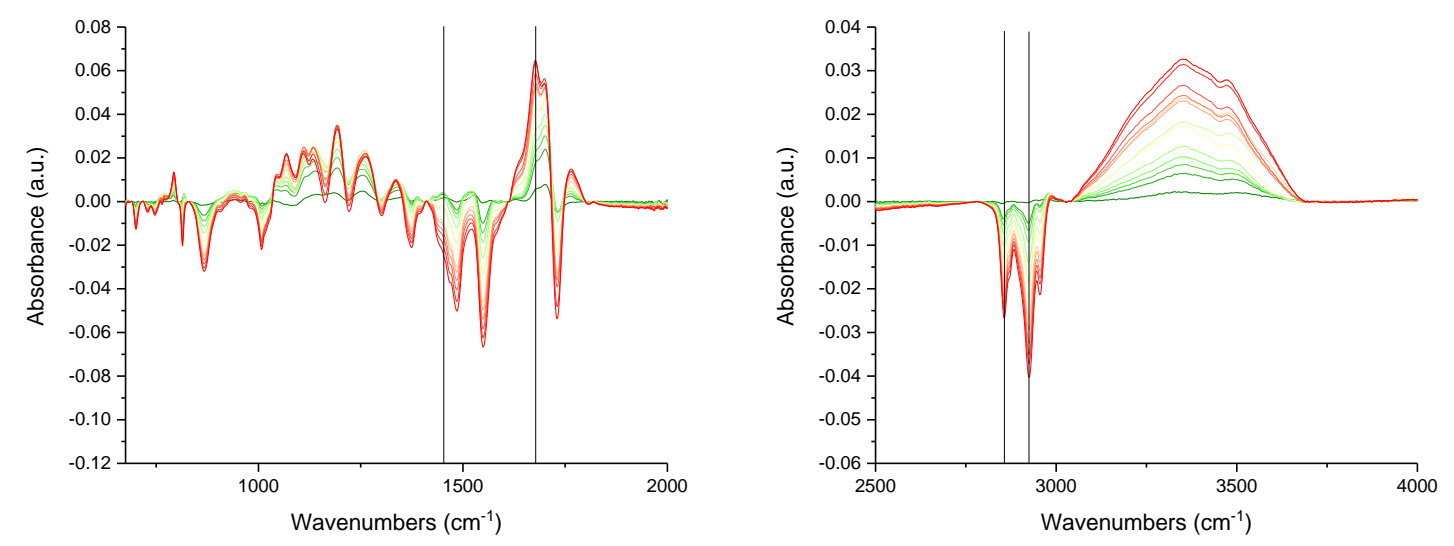

Figure 4.9 Subtraction spectra of a BASF R10CG392D sample after 1000 hours of weathering. Lines indicate the peaks used to track oxidation (left) and mass loss (right).

To compare the spectrometers, the degradation rates obtained from the macroscopic instrument were subtracted from those obtained from the microscopic instrument (Table 4.3). The rates of mass loss determined from the macroscopic instrument appear to be consistently lower (negative difference) than the rates determined by the microscopic instrument. The rate discrepancies are due to several factors, primarily differences in the working mechanisms of the instruments. The ATR crystals, detectors, and differences in the collection parameters all factor into the degradation rate discrepancies. The smaller sampling area of the microscopic instrument amplifies differences in degradation at each location. Therefore, comparisons of the degradation rates of different coatings require use of the same instrument. 
Table 4.3 Degradation Rate Differences between Instruments

\begin{tabular}{l|cc|cr} 
& Mass Loss & & Oxidation & \\
\cline { 2 - 5 } & Rate $\mathbf{( 1 0 ^ { - 5 } \mathbf { ~ m } ^ { \mathbf { 2 } } / \mathbf { M J } )}$ & error $\left(10^{-6}\right)$ & Rate $\left(\mathbf{1 0 ^ { - 5 }} \mathbf{~ m}^{\mathbf{2}} / \mathbf{M J}\right)$ & error $\left(10^{-6}\right)$ \\
\hline BEB & $\mathbf{- 0 . 7 4}$ & 1.2 & $\mathbf{- 1 2 . 0 2}$ & -8.03 \\
BEF & $\mathbf{- 1 . 3 8}$ & 4.9 & $\mathbf{- 2 4 . 4 0}$ & -24.23 \\
BEW & $\mathbf{0 . 0 8}$ & 0.9 & $\mathbf{- 1 3 . 1 5}$ & -10.54 \\
\hline BRB & $\mathbf{- 3 . 0 1}$ & -1.9 & $\mathbf{2 7 . 0 0}$ & -2.55 \\
BRF & -5.26 & -2.0 & $\mathbf{2 5 . 0 0}$ & -11.68 \\
BRW & $\mathbf{- 3 . 0 7}$ & -4.8 & $\mathbf{1 2 . 0 0}$ & 8.22 \\
\hline PAB & $\mathbf{- 1 . 7 6}$ & 2.3 & $\mathbf{1 4 . 1 5}$ & 2.11 \\
PAW & $\mathbf{- 1 . 7 0}$ & -0.4 & $\mathbf{2 0 . 5 6}$ & -7.93 \\
\hline PNB & $\mathbf{0 . 1 8}$ & -0.5 & $\mathbf{- 1 . 3 5}$ & -0.25 \\
PNW & $\mathbf{0 . 5 1}$ & -0.8 & $\mathbf{1 0 . 2 0}$ & -9.44 \\
\hline PTB & $\mathbf{- 1 . 0 9}$ & 0.8 & $\mathbf{5 . 4 8}$ & 16.63 \\
PTW & $\mathbf{- 1 . 6 3}$ & -0.8 & $\mathbf{1 2 . 2 9}$ & -13.39
\end{tabular}

The effect of sample color on mass loss and oxidation rates was more consistent (Table 4.4). In general, samples with black base coats degraded faster via both mass loss and oxidation than samples of the same chemistry with white base coats. Exceptions to this were the BASF E10CG081G and PPG TMAC coatings. The BASF free films both degraded faster than their full system counterparts. This is could be due to the addition of UV stabilizers to the base coats by some manufacturers, providing a reservoir to extend degradation-resistance of the clear coat.

Table 4.4 Degradation Rate Differences by Color and Chemistry

\begin{tabular}{|c|c|c|c|c|c|c|}
\hline & \multicolumn{3}{|l|}{ Mass Loss } & \multicolumn{3}{|l|}{ Oxidation } \\
\hline & Rate $\left(10^{-5} \mathrm{~m}^{2} / \mathrm{MJ}\right)$ & error $\left(10^{-6}\right)$ & $R^{2}$ & Rate $\left(10^{-5} \mathrm{~m}^{2} / \mathrm{MJ}\right)$ & error $\left(10^{-6}\right)$ & $\mathrm{R}^{2}$ \\
\hline BEB & -5.12 & 3.2 & 0.95 & 24.55 & 7.6 & 0.99 \\
\hline BRB & -11.13 & 1.8 & 1.00 & 131.00 & 24.4 & 1.00 \\
\hline PAB & -15.29 & 6.9 & 0.98 & 61.94 & 31.6 & 0.97 \\
\hline PNB & -5.97 & 5.8 & 0.93 & 61.52 & 31.4 & 0.98 \\
\hline PTB & -5.96 & 4.2 & 0.94 & 65.79 & 57.2 & 0.90 \\
\hline BEW & -6.58 & 7.3 & 0.88 & 30.45 & 12.5 & 0.98 \\
\hline BRW & -8.67 & 2.2 & 0.99 & 122.00 & 50.6 & 0.98 \\
\hline PAW & -14.86 & 3.1 & 0.99 & 46.13 & 12.0 & 0.99 \\
\hline PNW & -5.40 & 2.3 & 0.97 & 46.14 & 22.5 & 0.96 \\
\hline PTW & -6.70 & 2.4 & 0.98 & 87.83 & 26.1 & 0.99 \\
\hline BEF & -8.66 & 8.8 & 0.91 & 30.89 & 11.0 & 0.99 \\
\hline BRF & -17.32 & 5.4 & 0.99 & 168.00 & 42.0 & 0.99 \\
\hline
\end{tabular}


For the black samples, rates of mass loss in descending order were PPG APO, BASF R10CG392D, PPG NCT, PPG TMAC, and BASF E10CG081G. For the white samples, rates of mass loss in descending order were PPG APO, BASF R10CG392D, PPG TMAC, BASF E10CG081G, and PPG NCT. Mass loss is typically due to chain scission and backbone fragmentation. Since mass loss is a general indicator of degradation, the different rates may be due to differences in the specific structure of the acrylic backbones. The oxidation rates by chemistry in descending order were BASF R10CG392D, PPG TMAC, PPG APO, PPG NCT, and BASF E10CG081G for the black samples, and BASF R10CG392D, PPG TMAC, PPG NCT, PPG APO, and BASF E10CG081G for the white samples. The major chemical difference between the fastest (BASF R10CG392D) and slowest (PPG APO and BASF E10CG081G) oxidizing samples was the absence of urethane crosslinking in the fastest degrading samples. The latter two samples were new generation "oxithane"-type acrylic urethane coatings, which may be more resistant to photooxidation.

Based on the raw MSP data, the sample set contained only benzotriazoletype UVAs, which exhibit characteristic absorptions at roughly $230 \mathrm{~nm}$ and $340 \mathrm{~nm}$ (Figure 4.10). ${ }^{77}$ The UVAs used in the BASF clear coats were confirmed by manufacturer information (Figure 4.11). Since the UVAs used in the reference film and 
the sample set were also of benzotriazole class, the peak at $345 \mathrm{~nm}$ was used to calculate TCA for all the samples.

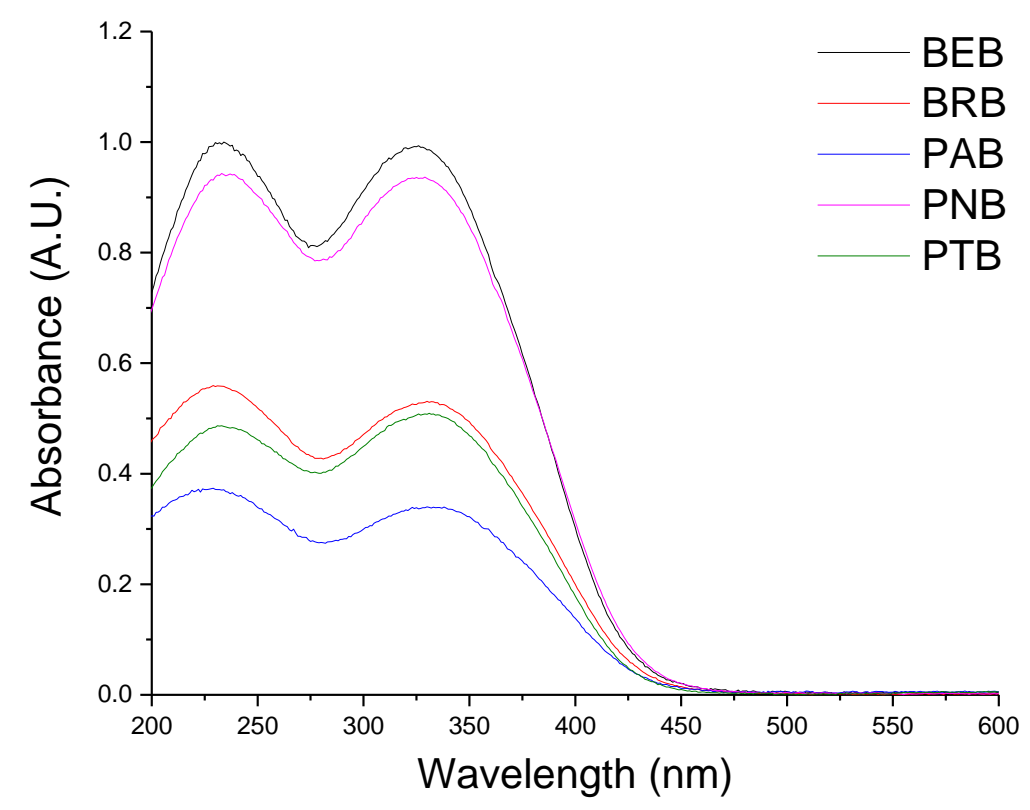

Figure 4.10 MSP spectra of unweathered samples.

(a)

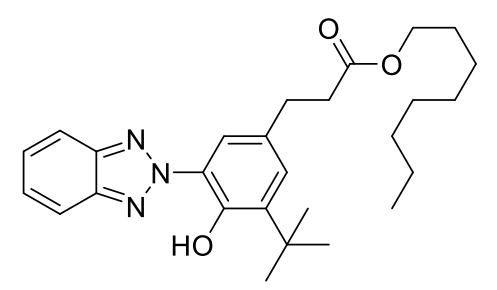

(b)

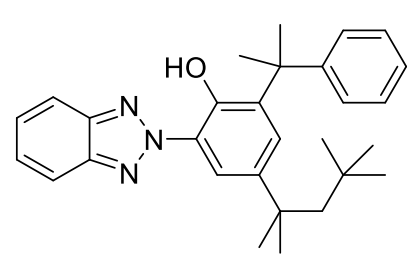

Figure 4.11 Structures of the benzotriazole UVAs used in the BASF clear coats (a) E10CG081G and (b) R10CG392D.

TCA for all the samples decreased with UV exposure as anticipated (Figure 4.12). The acrylic urethane PPG APO coating was the only chemistry to exhibit consistent absorbance decrease between colors. The rates of change determined by linear regression are shown in Table 4.5. In general, TCA of the black samples decreased faster than the white samples. This was likely because black absorbs light more effectively than white, resulting in faster UVA depletion. 

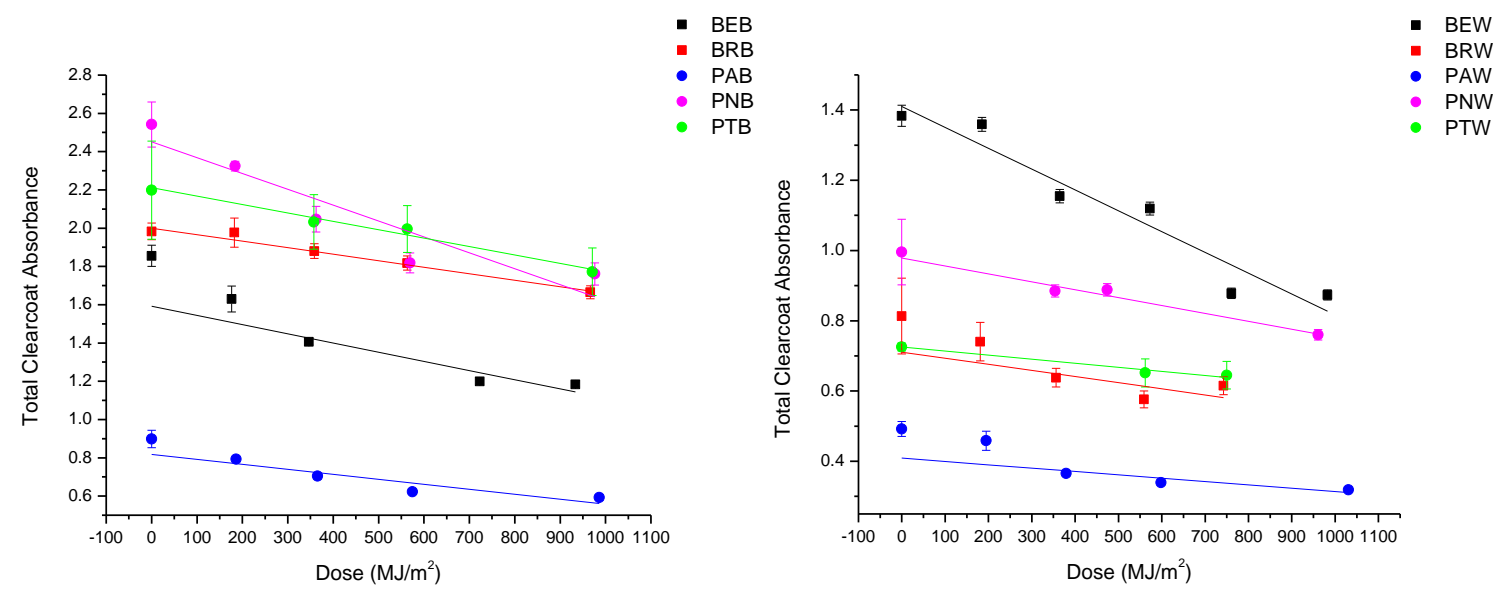

Figure 4.12 Total clearcoat absorbance with UV exposure of black (left) and white (right) samples.

Table 4.5 Rates of Total Clearcoat Absorbance Decline

\begin{tabular}{lccc} 
Sample & Rate $\left(\mathbf{1 0 ^ { - 5 }} \mathbf{~}^{\mathbf{2}} / \mathbf{M J}\right)$ & Error $\left(10^{-6}\right)$ & $R^{2}$ \\
\hline BEB & $\mathbf{- 4 7 . 9 3}$ & 125.0 & 0.91 \\
BRB & $\mathbf{- 3 3 . 9 5}$ & 21.9 & 0.99 \\
PAB & $\mathbf{- 2 5 . 9 4}$ & 64.0 & 0.92 \\
PNB & $\mathbf{- 8 2 . 9 1}$ & 178.0 & 0.94 \\
PTB & $\mathbf{- 4 3 . 9 8}$ & 54.3 & 0.99 \\
BEW & $\mathbf{- 5 9 . 3 4}$ & 92.2 & 0.95 \\
BRW & $\mathbf{- 1 7 . 3 9}$ & 110.4 & 0.67 \\
PAW & -9.53 & 35.6 & 0.84 \\
PNW & $\mathbf{- 2 2 . 5 5}$ & 30.3 & 0.98 \\
PTW & $\mathbf{- 1 1 . 5 8}$ & 12.5 & 0.99
\end{tabular}

Color change was recorded as a physical indicator of weathering. Change in yellowing index, $b^{*}$, and $\mathrm{DE}^{*}$ were calculated. The change in yellowing index for the BASF and PPG clear coats with increasing dose are shown in Figure 4.13. The error bars are the standard error calculated with Equation 6. The BASF R10CG392D sample (BRW) yellowed faster than the other chemistries, in agreement with the degradation rates determined by FTIR spectroscopy. The two "oxithane"-type urethane samples (BEW and PAW) did not exhibit the same yellowing 
trends despite similar chemistries, as yellowing of the PPG APO sample plateaued at roughly $800 \mathrm{MJ} / \mathrm{m}^{2}$ dose.
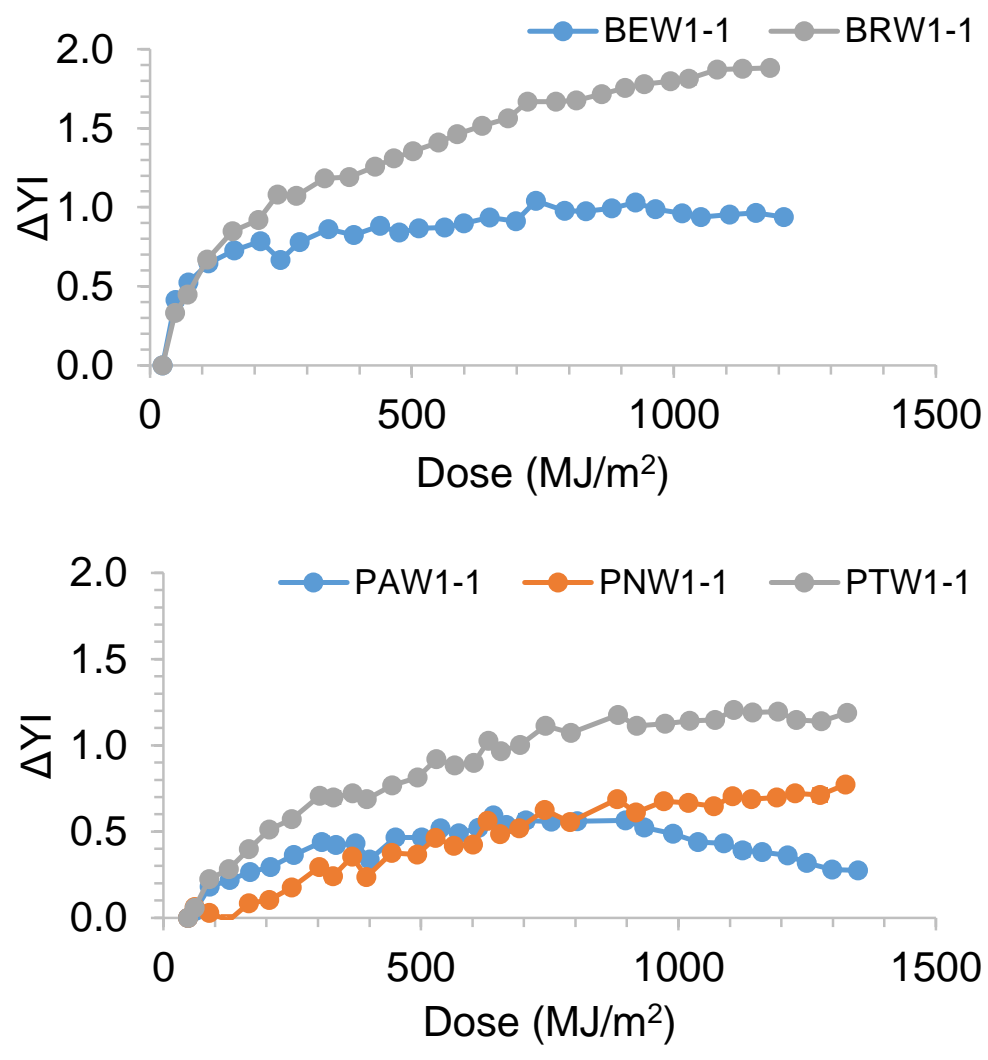

Figure 4.13 Change in YI with dose of BASF (top) and PPG (bottom) samples. Error bars represent the standard variance. Lines are eye guides.

Within the same clear coat type, error in $\mathrm{YI}$ measurements was always greater for the black samples (data not shown) than the white samples. This is because the ASTM color standard for $\mathrm{Yl}$ is based on white or near-white objects. ${ }^{80}$ Therefore, change in $b^{*}$ was used to compare yellowing between the white and black samples, as yellowing should manifest as an increase in $b^{*}$ with dose (Figure 4.14). The black samples exhibited yellowing as $b^{*}$ increased with dose, but this change was not as pronounced as the increase observed in the white samples. 
However, this method of comparing the yellowing of non-white samples is still preferred over $\mathrm{YI}$ change, as $\mathrm{YI}$ was designed for white objects.
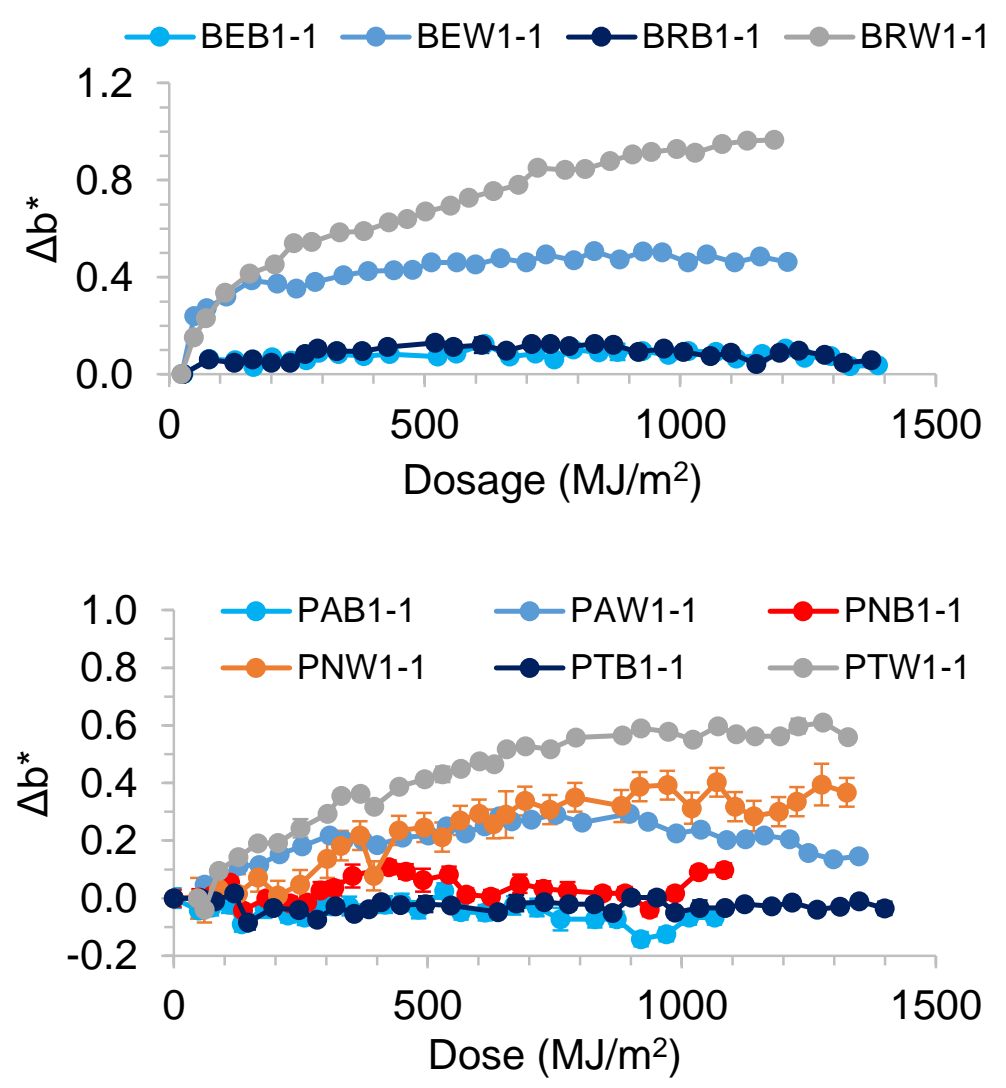

Figure 4.14 Change in $b^{*}$ with dose of BASF (top) and PPG (bottom) samples. Error bars represent the standard variance. Lines are eye guides.

Weathering induces overall color changes as well, affecting lightness or $L^{*}$, so tracking $\mathrm{DE}^{*}$ with dose using the initial and weathered color measurements provides a more general gauge of color change (Figure 4.15). The same trend is seen where the $\mathrm{DE}^{*}$ of the white samples changed with dose faster than the black samples; for example, $\mathrm{DE}^{\star}$ of $\mathrm{BEW}$ increased faster than $\mathrm{BEB}$. If $\mathrm{DE}^{*}$ is considered a physical indicator of chemical degradation, this would mean clear coats on white base coats undergo photodegradation faster than on black base coats under the 
same conditions. However, this is contrary to the concept that black absorbs UV radiation more readily than white. This is likely because gloss and texture also impact color measurements as higher gloss specimens will tend to be detected as darker. In terms of clear coat chemistry, the white PPG NCT and TMAC samples exhibit similar color change trends. These were the only two chemistries not urethane crosslinked, so they may undergo similar degradative pathways.
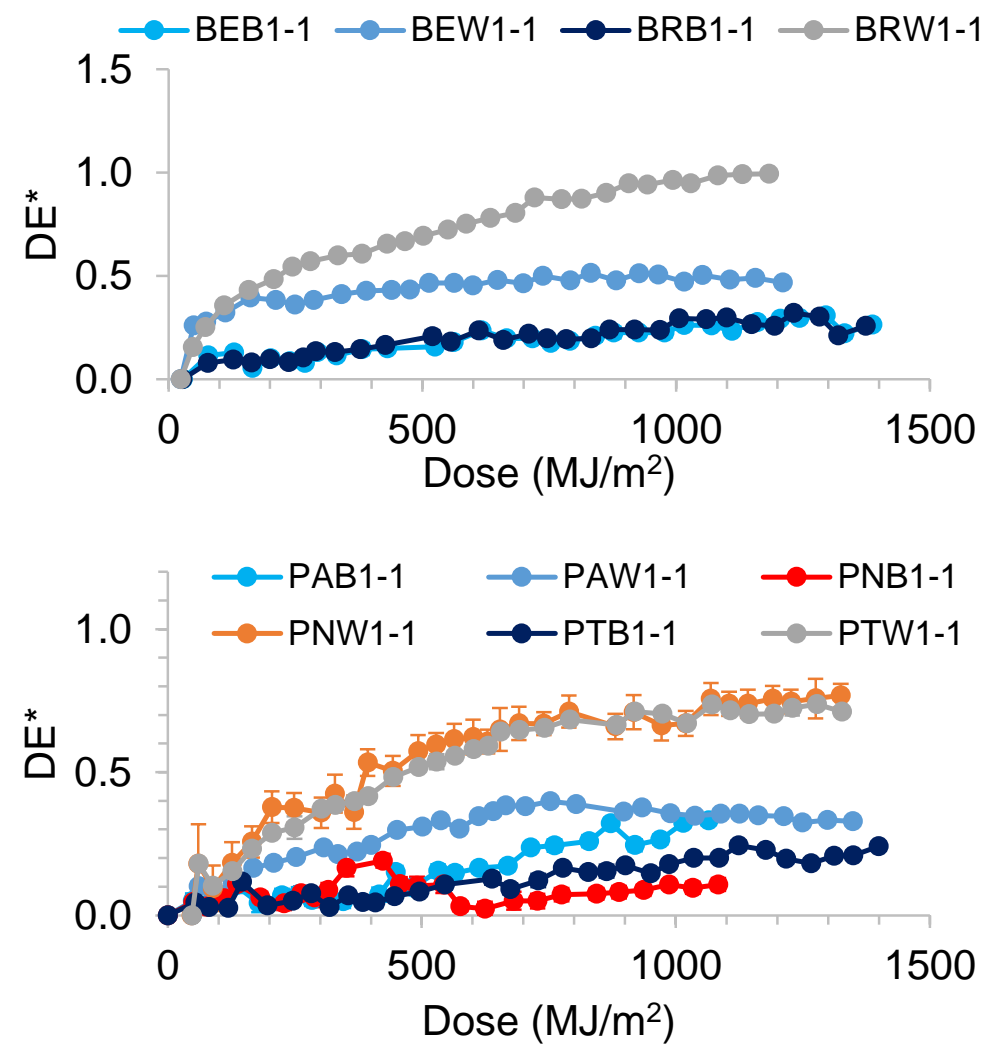

Figure 4.15 DE* between initial and weathered BASF (top) and PPG (bottom) samples. Error bars represent the standard variance. Lines are eye guides.

Gloss did not change with weathering as significantly as color based on $20^{\circ}$ (Figure 4.16) or $60^{\circ}$ gloss retention. Although a slight decrease can be qualitatively observed, the error in the measurements does not permit quantitation of gloss de- 
cline. Trends in the effects of sample chemistry and color are similar to those observed in the color measurements. Gloss decreased sooner for black samples than white based on the PPG TMAC samples (PTB and PTW). This is likely due to the superior ability of black samples to absorb heat, accelerating photodegradation reactions. Within the black samples, the more rapid gloss decline of the TMAC sample compared to the other two PPG samples may be due to the metallic flakes causing the coating to absorb and retain heat more effectively.
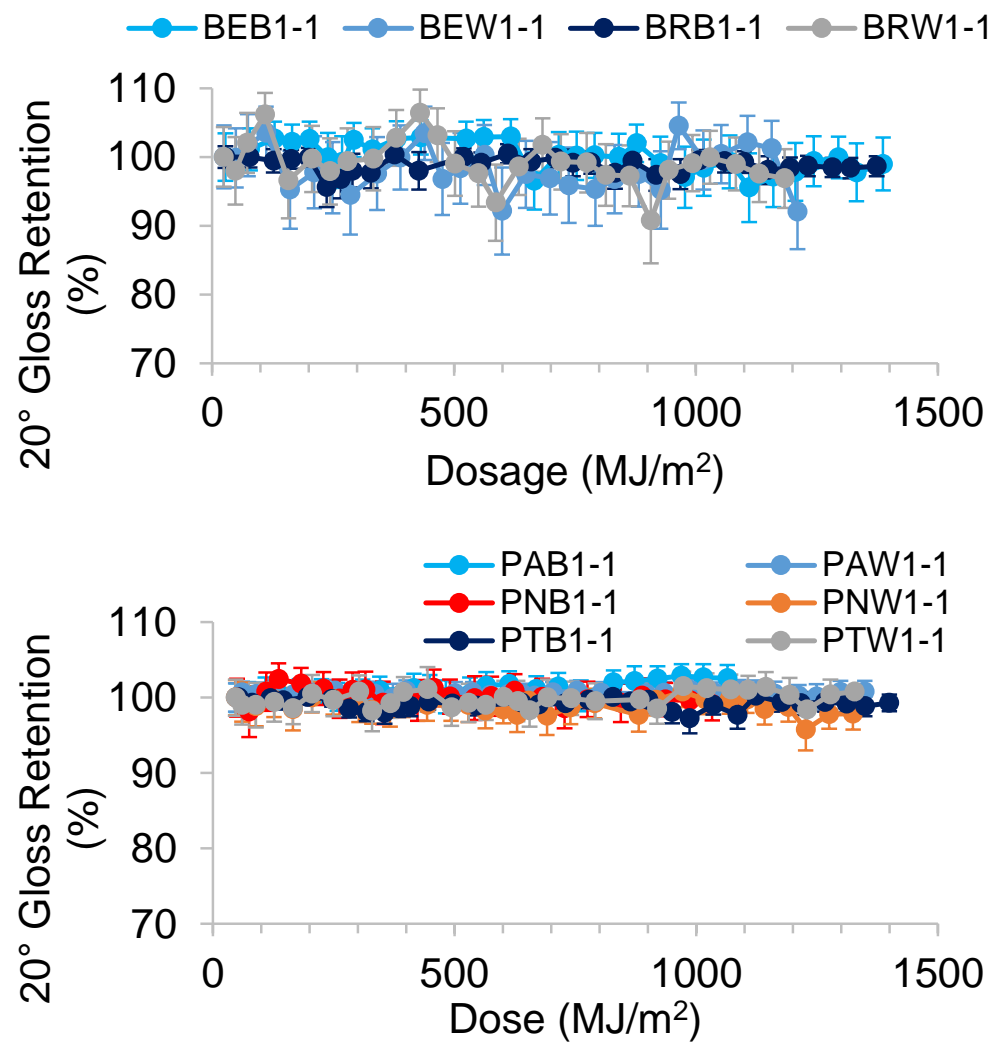

Figure 4.16 Gloss retention with increasing dose of BASF (top) and PPG (bottom) clear coats. Error bars represent the standard variance. Lines are eye guides. 


\subsection{Conclusions}

Accelerated weathering experiments can provide insights on the physical and chemical changes automotive clear coats undergo during exposure. This may allow forensic examiners of such trace evidence to more accurately gauge the age and history of a sample. The three acrylic urethane and two acrylic melamine clear coats weathered in this study exhibited significant chemical degradation via chain scission and photo-oxidation characterized by ATR FTIR and Raman spectroscopies. These samples also exhibited significant physical degradation via yellowing and general color change, though $20^{\circ}$ gloss did not decrease as much. The acrylic urethane "oxithane"-type clear coats chemically degraded slower than the other clear coats, while the acrylic melamine clear coats exhibited the fastest color change. In general, the clear coats on black base coats degraded faster than on white, and the free films degraded the fastest. Further work should focus on replicating and confirming these results, and analysis of chemistries from the third major automotive coatings manufacturer in North America. 


\section{Final Remarks}

The objective of this work was to develop procedures to make forensic examinations more quantitative in order to improve specific vehicle identification. Color measurement and spectroscopic methods employed by automotive manufacturers and the forensics community were examined to progress the development of procedures for quantitative measurements of paints. Additionally, initial weathering experiments were performed on unexposed OEM coatings to gauge uncertainties in physical and chemical characterization measurements and improve current protocols for accelerated exposure experiments.

Master standards and reference or control panels are characterized by manufacturers using color meters, so relating microscopic forensics color measurements to colorimeter values may supply examiners with additional information on questioned samples. These results provide an initial gauge of the variation in color differences between two samples as measured by color meter and MSP in reflectance and transmission modes. Since spectroscopy is predominantly employed for chemical characterization of paints in the forensics community, transmission FTIR and Raman microspectroscopic methods were investigated with a focus on improving sample preparation ease and repeatability. This was accomplished by microtoming cross sections of the embedded samples so the all layers could be analyzed with a single preparation of consistent thickness. Finally, auto-

motive original equipment manufacturer (OEM) paints were artificially weathered and their clear coats characterized with microscopic instrumentation methods to 
gauge the utility for forensic examinations aimed at obtaining vehicle history information. The chemical data available from microscopic clear coat analyses such as MSP for UVA loss may be useful for determining specific vehicle of origin when a proper control is unavailable. In general, these results should improve the colorimetric and chemical forensic characterizations of paints by the forensics community for specific vehicle identification. 


\section{REFERENCES}

(1) Coleman, J.; Barrett, R.; Rector, S. Handbook of Forensic Servies. FBI Laboratory: Quantico, Virginia 2013, p 63.

(2) Federal Bureau of Investigation. Quick Reference Guide for Laboratory Examinations. 2016, p 9.

(3) Wright, D. Forensic Paint Examination: Using Automotive Paint Sourcing to Solve Crimes. FBI Law Enforcement Bulletin. September 3, 2013.

(4) Greenaway, K. Comments to the National Commission on Forensic Science Submitted By Keith Greenaway; 2014.

(5) Justice, D. of. NATIONAL COMMISSION ON FORENSIC SCIENCE https://www.justice.gov/archives/ncfs.

(6) Gillerman, G.; Merkel, W.; Reczek, K. Standards Training \& Discussion; 2015.

(7) Scientific Working Group for Materials Analysis (SWGMAT). Forensic Paint Analysis and Comparison Guidelines; 2000.

(8) Causin, V. Polymers on the crime scene: Forensic analysis of polymeric trace evidence; Springer, 2015.

(9) Covestro. PU automotive OEM coatings deliver aesthetics and performance http://www.coatings.covestro.com/Applications/AutomotiveTransportation/OEM-Metal.aspx.

(10) Ryland, S. G. Infrared Microspectroscopy of Forensic Paint Evidence. In Practical Guide to Infrared Microscpectroscopy; Marcel Dekker, Inc., 1995; pp 185-189.

(11) ASTM International. Standard Guide for Forensic Paint Analysis and Comparison. E1610-14. 2014.

(12) X-Rite Incorporated. Ways to Measure Color; 2007. 
(13) Klein, G. A. Industrial Color Difference. In Industrial Color Physics; Springer, 2010; pp 133-163.

(14) ASTM International. Standard Practice for Identification of Instrumental Methods of Color or Color Difference Measurement of Materials. E805-12a. 2012.

(15) ASTM International. Standard Practice for Obtaining Spectrometric Data for Object-Color Evaluation. E1164-12. 2012.

(16) ASTM International. Standard Practice for Establishing Color and Gloss Tolerances. D3134-15. 2015.

(17) Scientific Working Group for Materials Analysis (SWGMAT). Standard Guide for Microspectrophotometry and Color Measurement in Forensic Paint Analysis; 2007.

(18) Trzcińska, B.; Zieba-Palus, J.; Kościelniak, P. Application of microspectrometry in the visible range to differentiation of car paints for forensic purposes. J. Mol. Struct. 2009, 924-926 (C), 393-399 DOI: 10.1016/j.molstruc.2008.10.037.

(19) Zięba-Palus, J.; Trzcińska, B.; Kościelniak, P. Comparative Analysis of Car Paint Traces in Terms of Color by VIS Microspectrometry for Forensic Needs. Anal. Lett. 2010, 43 (3), 436-445 DOI: $10.1080 / 00032710903402358$.

(20) Zięba-Palus, J.; Trzcińska, B. M. Comparing the Color of Forensic Traces. Anal. Lett. 2012, 45 (11), 1333-1346 DOI: $10.1080 / 00032719.2012 .675483$.

(21) International Commission on Illumination. Improvement To Industrial Colour-Difference Evaluation; 2001.

(22) Simon, R. Chemical Analysis and Databases in the Paint Industry. Valspar 2016.

(23) BYK-Gardner Inc. Total Color Impression of Effect Coatings Measurement of Fluorescence. 2013, pp 2-25. 
(24) ASTM International. Standard Test Method for Multiangle Color Measurement of Metal Flake Pigmented Materials. E2194-14. 2014.

(25) ASTM International. Standard Test Method for Multiangle Color Measurement of Interference Pigments. E2539-14. 2014.

(26) Kopchick, K. A.; Bommarito, C. R. Color analysis of apparently achromatic automotive paints by visible microspectrophotometry. J. Forensic Sci. 2006, 51 (2), 340-343 DOI: 10.1111/j.1556-4029.2006.00050.x.

(27) ASTM International. Examination of Paint Review: 2010 to 2013; 2013.

(28) Trzcinska, B.; Zieba-Palus, J.; Koscielniak, P. Examination of Car Paint Samples Using Visible Microspectrometry for Forensic Purposes. Anal. Lett. 2013, 46 (February), 1267-1277 DOI: Doi 10.1080/00032719.2012.760099.

(29) BYK-Gardner Inc. spectro-guide. 2013, p 79.

(30) ASTM International. Standard Practice for Computing the Colors of Objects by Using the CIE System. E308-15. 2015.

(31) Nichols, M. Paint Materials and Processes from an Automotive OEM Perspective. Ford 2016.

(32) Vik, M.; Cejka, V.; Vikova, M.; Maltseva, A. Color Appearance of Decorative Coatings. Proc. 21st Int. Conf. Light SVËTLO 2015 2015, 281284 DOI: 10.13164/conf.light.2015.281.

(33) Chen, R.; Lv, J.; Feng, J. Characterization of Paint by Fourier-Transform Infrared Spectroscopy, Raman Microscopy, and Scanning Electron Microscopy-Energy Dispersive X-ray Spectroscopy. Anal. Lett. 2014, 48 (9), 1502-1510 DOI: 10.1080/00032719.2014.984190.

(34) ASTM International. Infrared Spectra of U.S. Automobile Original Finishes (Post - 1989). VIII: In Situ Identification of Bismuth Vanadate Using Extended Range FT-IR Spectroscopy, Raman Spectroscopy, and X-Ray Fluorescence Spectrometry. J. Forensic Sci. 2014, 59 (2), 344-363 DOI: 10.1111/1556-4029.12318. 
(35) Society of Dyers and Colourists. Definitions of a dye and a pigment http://www.colour-index.com/definitions-of-a-dye-and-a-pigment.

(36) Zięba-Palus, J.; Borusiewicz, R. Examination of multilayer paint coats by the use of infrared, Raman and XRF spectroscopy for forensic purposes. $J$. Mol. Struct. 2006, 792-793, 286-292 DOI:

10.1016/j.molstruc.2006.03.072.

(37) Suzuki, E. M. Infrared spectra of U.S. automobile original topcoats (19741989): II. Identification of some topcoat inorganic pigments using an extended range $(4000-220 \mathrm{~cm}-1)$ fourier transform spectrometer. J. Forensic Sci. 1996, 41 (3), 393-406.

(38) Suzuki, E. M. Infrared Spectra of U . S . Automobile Original Topcoats ( 1974-1989 )" I1 . Identification of Some Topcoat Inorganic Pigments Using an Extended Range ( 4000-220 cm -1 ) Fourier Transform Spectrometer. $J$ Forensic Sci 1996, 393-406 DOI: 10.1520/JFS13924J.

(39) Lv, J. G.; Liu, S.; Feng, J. M.; Liu, Y.; Zhou, S. D.; Chen, R. Effective identification of paints pigments in hit-and-run cases with confocal Raman microscope. Pigment Resin Technol. 2016, 45 (4), 294-300 DOI: 10.1108/PRT-05-2015-0044.

(40) Lv, J.; Zhang, W.; Liu, S.; Chen, R.; Feng, J.; Zhou, S.; Liu, Y. Analysis of 52 automotive coating samples for forensic purposes with Fourier transform infrared spectroscopy (FTIR) and Raman microscopy. Environ. Forensics 2016, 17(1), 59-67 DOI: 10.1080/15275922.2015.1091403.

(41) Zieba-Palus, J.; Trzcińska, B. M. Application of infrared and raman spectroscopy in paint trace examination. J. Forensic Sci. 2013, 58 (5), 1359-1363 DOI: 10.1111/1556-4029.12183.

(42) Lv, J.; Feng, J.; Liu, Y.; Wang, Z.; Zhao, M.; Cai, Y.; Shi, R. Discriminating paints with different clay additives in forensic analysis of automotive coatings by FT-IR and Raman spectroscopy. Spectroscopy 2012, 27 (4), 36-43.

(43) Zięba-Palus, J.; Michalska, A. Characterization of blue pigments used in automotive paints by raman spectroscopy. J. Forensic Sci. 2014, 59 (4), 943-949 DOI: 10.1111/1556-4029.12499. 
(44) Skenderovska, M.; Minčeva-Šukarova, B.; Andreeva, L. Application of Micro-Raman and Ft-Ir Spectroscopy in Forensic Analysis of. Maced. J. Chem. Chem. Eng. 2008, 27 (1), 9-17.

(45) De Gelder, J.; Vandenabeele, P.; Govaert, F.; Moens, L. Forensic analysis of automotive paints by Raman spectroscopy. J. Raman Spectrosc. 2005, 36 (11), 1059-1067 DOI: 10.1002/jrs.1408.

(46) Maric, M.; van Bronswijk, W.; Lewis, S. W.; Pitts, K. Rapid characterisation and classification of automotive clear coats by attenuated total reflectance infrared spectroscopy. Anal. Methods 2012, 4 (9), 2687 DOI: 10.1039/c2ay25419k.

(47) Sauzier, G.; Maric, M.; van Bronswijk, W.; Lewis, S. W. Preliminary studies into the effect of environmental degradation on the characterisation of automotive clear coats by attenuated total reflectance infrared spectroscopy. Anal. Methods 2013, 5, 4984-4990.

(48) Lavine, B. K.; Fasasi, A.; Mirjankar, N.; Nishikida, K.; Campbell, J. Simulation of attenuated total reflection infrared absorbance spectra: applications to automotive clear coat forensic analysis. Appl. Spectrosc. 2014, 68 (5), 608-615 DOI: 10.1366/13-07266.

(49) Scientific Working Group for Materials Analysis (SWGMAT). Washington State Crime Laboratory Infrared Spectral Library of Automotive Paint Pigments.

(50) ThermoFisher Scientific. Coatings Technology FT-IR Spectral Library https://www.thermofisher.com/order/catalog/product/834-010901M.

(51) Royal Canadian Mounted Police. Paint Data Query (PDQ) http://www.rcmp-grc.gc.ca/fsis-ssji/paint-peinture-eng.htm.

(52) Royal Canadian Mounted Police. Paint Data Query (PDQ). 2016, p 1.

(53) Fasasi, A.; Mirjankar, N.; Stoian, R. I.; White, C.; Allen, M.; Sandercock, M. P.; Lavine, B. K. Pattern recognition-assisted infrared library searching of automotive clear coats. Appl. Spectrosc. 2015, 69 (1), 84-94 DOI: 10.1366/14-07578. 
(54) Bradley, M. Analyzing Automotive Paints With Extended Range ATR is No Mystery https://www.thermofisher.com/blog/polymers2plastics/analyzingautomotive-paints-with-extended-range-atr-is-no-mystery/.

(55) Ryland, S.; Bishea, G.; Brun-Conti, L.; Eyring, M.; Flanagan, B.; Jergovich, T.; MacDougall, D.; Suzuki, E. Discrimination of 1990s original automotive paint systems: a collaborative study of black nonmetallic base coat/clear coat finishes using infrared spectroscopy. J. Forensic Sci. 2001, 46 (1), $31-45$.

(56) HORIBA. What analysis spot size is used for a Raman microscope? http://www.horiba.com/scientific/products/raman-spectroscopy/ramanacademy/raman-faqs/what-analysis-spot-or-laser-spot-size-is-used-for-araman-microscope/.

(57) Bruker Corporation. SENTERRA User Manual. p 13.

(58) Palenik, C. S.; Palenik, S.; Groves, E. Fundamentals of Forensic Pigment Identification by Raman Microspectroscopy: A Practical Identification Guide and Spectral Library for Forensic Science Laboratories; 2011.

(59) Maric, M.; van Bronswijk, W.; Pitts, K.; Lewis, S. W. Characterisation and classification of automotive clear coats with Raman spectroscopy and chemometrics for forensic purposes. J. Raman Spectrosc. 2016, No. April DOI: $10.1002 / j r s .4925$.

(60) Coleman, M. M.; Painter, P. C. Fundamentals of Polymer Science: An Introductory Text, 2nd ed.; CRC Press, 1998.

(61) HORIBA. Raman Spectroscopy for Analysis and Monitoring. HORIBA Jobin Yvon Raman Application Note.

(62) AnalyzingIT Inc. RAMAN Band Correlation Table. 2010.

(63) Nguyen, T.; Martin, J. W.; Byrd, E.; Embree, E.; Embree, E. Effects of Spectral UV on Degradation of Acrylic-Urethane Coatings. Proc. 80th Annu. Meet. Progr. Fed. Soc. Coatings Technol. LA, New Orleans 2002.

(64) Chmela, Š. Photo degradation of polymers. POLYFRIEND: Advanced Bio- 
Friendly Polymers 2013, p 6.

(65) Nechifor, M. Factors Influencing the Photochemical Behavior of Multicomponent Polymeric Materials. In Photochemical Behavior of Multicomponent Polymeric-based Materials; 2016; pp 21-65.

(66) Adema, K. N. S.; Makki, H.; Peters, E. A. J. F.; Laven, J.; Van Der Ven, L. G. J.; Van Benthem, R. A. T. M.; De With, G. The influence of the exposure conditions on the chemical and physical changes of polyester-urethane coatings during photodegradation. Polym. Degrad. Stab. 2016, 123, 13-25 DOI: 10.1016/j.polymdegradstab.2015.09.026.

(67) Nguyen, T.; Martin, J.; Byrd, E.; Embree, N. Relating laboratory and outdoor exposure of coatings III. Effect of relative humidity on moistureenhanced photolysis of acrylic-melamine coatings. Polym. Degrad. Stab. 2002, 77 (1), 1-16 DOI: 10.1016/S0141-3910(02)00070-8.

(68) Bauer, D. R.; Mielewski, D. F. The role of humidity in the photooxidation of acrylic melamine coatings. Polym. Degrad. Stab. 1993, 40 (3), 349-355 DOI: 10.1016/0141-3910(93)90141-5.

(69) Bauer, D. R. Global exposure models for automotive coating photooxidation. Polym. Degrad. Stab. 2000, 69 (3), 297-306 DOI: 10.1016/S0141-3910(00)00073-2.

(70) Bauer, D. R. Interpreting weathering acceleration factors for automotive coatings using exposure models. Polym. Degrad. Stab. 2000, 69 (3), 307316 DOI: 10.1016/S0141-3910(00)00074-4.

(71) Adema, K. N. S. Photodegradation of polyester-urethane coatings, Eindhoven University of Technology, 2015.

(72) Lemaire, J.; Siampiringue, N. Prediction of Coating Lifetime Based on FTIR Microspectrophotometric Analysis of Chemical Evolutions. In Service Life Prediction of Organic Coatings; 1999; pp 246-256.

(73) Gerlock, J. L.; Kucherov, A. V.; Nichols, M. E. On the Combined Use of UVA, HALS, Photooxidation, and Fracture Energy Measurements to Anticipate The Long-Term Weathering. J. Coatings Technol. 2001, 73 (918), 45-54. 
(74) Watson, S.; Tseng, I.; Forster, A.; Chin, J.; Sung, L. Investigating Pigment Photoreactivity for Coatings Applications: Methods Development. In Service Life Prediction of Polymeric Materials; 2009; pp 423-456.

(75) Adema, K. N. S.; Makki, H.; Peters, E. A. J. F.; Laven, J.; Van Der Ven, L. G. J.; Van Benthem, R. A. T. M.; De With, G. Depth-resolved infrared microscopy and UV-VIS spectroscopy analysis of an artificially degraded polyester-urethane clearcoat. Polym. Degrad. Stab. 2014, 110, 422-434 DOI: 10.1016/j.polymdegradstab.2014.10.004.

(76) Liszewski, E. A.; Lewis, S. W.; Siegel, J. A.; Goodpaster, J. V. Characterization of automotive paint clear coats by ultraviolet absorption microspectrophotometry with subsequent chemometric analysis. Appl. Spectrosc. 2010, 64 (10), 1122-1125 DOI: 10.1366/000370210792973631.

(77) Smith, C. A.; Gerlock, J. L.; Carter, R. O. Determination of ultraviolet light absorber longevity and distribution in automotive paint systems using ultraviolet micro-spectroscopy. Polym. Degrad. Stab. 2001, 72 (1), 89-97 DOI: 10.1016/S0141-3910(00)00207-X.

(78) Nichols, M.; Boisseau, J.; Pattinson, L. An improved accelerated weathering protocol to anticipate Florida exposure behavior of coatings. $J$. Coatings Technol. Res. 2013, 10 (2), 153-173 DOI: 10.1007/s11998-0129467-x.

(79) Gu, X.; Stanley, D.; Byrd, W. E.; Dickens, B.; Vaca-Trigo, I.; Meeker, W. Q.; Nguyen, T.; Chin, J. W.; Martin, J. W. Linking Accelerated Laboratory Test with Outdoor Performance Results for a Model Epoxy Coating System. In Service Life Prediction of Polymeric Materials; 2009; pp 3-28.

(80) ASTM International. Standard Practice for Calculating Yellowness and Whiteness Indices from Instrumentally Measured Color Coordinates. E31315. 2015.

(81) Nguyen, T.; Martin, J.; Byrd, E. Relating laboratory and outdoor exposure of coatings: IV. Mode and mechanism for hydrolytic degradation of acrylicmelamine coatings exposed to water vapor in the absence of UV light. $J$. Coatings Technol. 2003, 75 (941), 37-50 DOI: 10.1007/BF02720521.

(82) PPG Industries Inc. Clearcoats http://www.ppgautocoatings.com/Products/Clearcoats.aspx. 
(83) Chin, J.; Byrd, E.; Embree, N.; Garver, J.; Dickens, B.; Finn, T.; Martin, J. Accelerated UV weathering device based on integrating sphere technology. Rev. Sci. Instrum. 2004, 75 (11), 4951-4959 DOI: 10.1063/1.1808916.

(84) BYK-Gardner Inc. micro-gloss Manual. 2010, p 48.

(85) PerkinElmer. Atomic Spectroscopy: A Guide to Selecting the Appropriate Technique and System; 2013. 


\section{APPENDIX}

Table A.1 Average DE* (from Top to Bottom) Determined by Color Meter, R-MSP, and T-MSP

\begin{tabular}{|c|c|c|c|c|c|c|c|c|c|c|c|c|c|c|c|}
\hline & 12 & 13 & $18 \mathrm{~A}$ & $19 \mathrm{~A}$ & 34 & 35 & 34PS & 34PW & $10 P$ & 11 & 15 & 17 & $2 A$ & $58 \mathrm{P}$ & 59 \\
\hline \multirow[t]{3}{*}{12} & & 5 & 56 & 56 & 57 & 57 & 57 & 58 & 48 & 48 & 48 & 47 & 64 & 67 & 67 \\
\hline & & 2 & 39 & 35 & 32 & 31 & 30 & 34 & 21 & 20 & 21 & 18 & 49 & 32 & 48 \\
\hline & & 9 & 65 & 69 & 66 & 67 & 61 & 62 & 16 & 36 & 18 & 45 & 16 & 15 & 17 \\
\hline \multirow[t]{3}{*}{13} & & & 51 & 52 & 52 & 52 & 53 & 53 & 43 & 42 & 43 & 42 & 62 & 66 & 66 \\
\hline & & & 38 & 33 & 31 & 30 & 29 & 32 & 19 & 18 & 19 & 16 & 48 & 31 & 48 \\
\hline & & & 66 & 70 & 67 & 69 & 62 & 63 & 25 & 42 & 27 & 50 & 24 & 23 & 24 \\
\hline \multirow[t]{3}{*}{$18 \mathrm{~A}$} & & & & 0 & 1 & 2 & 3 & 3 & 19 & 19 & 18 & 18 & 57 & 64 & 66 \\
\hline & & & & 5 & 8 & 9 & 12 & 11 & 28 & 28 & 28 & 29 & 62 & 46 & 62 \\
\hline & & & & 6 & 6 & 7 & 10 & 12 & 65 & 55 & 64 & 54 & 58 & 62 & 65 \\
\hline \multirow[t]{3}{*}{$19 A$} & & & & & 1 & 2 & 3 & 3 & 19 & 19 & 18 & 18 & 58 & 64 & 67 \\
\hline & & & & & 4 & 4 & 9 & 9 & 23 & 23 & 23 & 24 & 59 & 42 & 59 \\
\hline & & & & & 5 & 6 & 10 & 10 & 68 & 60 & 67 & 59 & 61 & 65 & 68 \\
\hline \multirow[t]{3}{*}{34} & & & & & & $\overline{1}$ & 2 & 3 & 19 & 19 & 19 & 19 & 57 & 64 & 66 \\
\hline & & & & & & 4 & 6 & 7 & 21 & 21 & 21 & 22 & 57 & 40 & 57 \\
\hline & & & & & & 8 & 7 & 7 & 66 & 59 & 65 & 59 & 59 & 62 & 65 \\
\hline \multirow[t]{3}{*}{35} & & & & & & & 1 & 2 & 20 & 20 & 20 & 20 & 57 & 64 & 66 \\
\hline & & & & & & & 8 & 9 & 20 & 20 & 20 & 22 & 58 & 40 & 58 \\
\hline & & & & & & & 10 & 12 & 65 & 56 & 64 & 56 & 58 & 62 & 65 \\
\hline \multirow[t]{3}{*}{ 34PS } & & & & & & & & 1 & 21 & 21 & 21 & 21 & 57 & 64 & 67 \\
\hline & & & & & & & & 5 & 21 & 20 & 21 & 20 & 52 & 35 & 52 \\
\hline & & & & & & & & 4 & 60 & 55 & 60 & 56 & 54 & 56 & 59 \\
\hline \multirow[t]{3}{*}{ 34PW } & & & & & & & & & 22 & 22 & 22 & 22 & 57 & 64 & 67 \\
\hline & & & & & & & & & 25 & 25 & 25 & 24 & 54 & 38 & 54 \\
\hline & & & & & & & & & 63 & 58 & 62 & 59 & 56 & 58 & 61 \\
\hline \multirow[t]{3}{*}{ 10P } & & & & & & & & & & 2 & 2 & 3 & 58 & 64 & 65 \\
\hline & & & & & & & & & & 1 & 1 & 8 & 54 & 34 & 53 \\
\hline & & & & & & & & & & 28 & 4 & 38 & 8 & 10 & 13 \\
\hline \multirow[t]{3}{*}{11} & & & & & & & & & & & 1 & 1 & 56 & 62 & 64 \\
\hline & & & & & & & & & & & 1 & 7 & 53 & 33 & 52 \\
\hline & & & & & & & & & & & 25 & 10 & 27 & 36 & 40 \\
\hline \multirow[t]{3}{*}{15} & & & & & & & & & & & & 0 & 55 & 62 & 63 \\
\hline & & & & & & & & & & & & 8 & 53 & 34 & 53 \\
\hline & & & & & & & & & & & & 34 & 9 & 14 & 17 \\
\hline \multirow[t]{3}{*}{17} & & & & & & & & & & & & & 55 & 61 & 63 \\
\hline & & & & & & & & & & & & & 46 & 26 & 45 \\
\hline & & & & & & & & & & & & & 36 & 45 & 49 \\
\hline \multirow[t]{3}{*}{$2 A$} & & & & & & & & & & & & & & 7 & 10 \\
\hline & & & & & & & & & & & & & & 19 & 4 \\
\hline & & & & & & & & & & & & & & 9 & 13 \\
\hline \multirow[t]{2}{*}{$58 \mathrm{P}$} & & & & & & & & & & & & & & & 4 \\
\hline & & & & & & & & & & & & & & & $\begin{array}{c}19 \\
4\end{array}$ \\
\hline
\end{tabular}


Table A.2 Standard Deviation of DE* (from Top to Bottom) Determined by Color Meter, R-MSP, and T-MSP

\begin{tabular}{|c|c|c|c|c|c|c|c|c|c|c|c|c|c|c|c|}
\hline & 12 & 13 & $18 \mathrm{~A}$ & $19 A$ & 34 & 35 & 34PS & $34 \mathrm{PW}$ & $10 \mathrm{P}$ & 11 & 15 & 17 & $2 \mathrm{~A}$ & $58 \mathrm{P}$ & 59 \\
\hline \multirow[t]{3}{*}{12} & & 0.4 & 0.2 & 0.2 & 0.1 & 0.2 & 0.1 & 0.2 & 0.1 & 0.2 & 0.1 & 0.1 & 0.2 & 0.1 & 0.3 \\
\hline & & 0.3 & 2.1 & 2.2 & 2.1 & 1.7 & 2.1 & 3.0 & 0.3 & 0.5 & 0.4 & 0.3 & 1.7 & 0.5 & 2.7 \\
\hline & & 1.5 & 0.7 & 1.1 & 0.7 & 1.0 & 1.6 & 1.0 & 1.0 & 0.9 & 0.9 & 1.1 & 1.1 & 1.2 & 1.1 \\
\hline \multirow[t]{3}{*}{13} & & & 0.4 & 0.4 & 0.4 & 0.3 & 0.4 & 0.3 & 0.4 & 0.4 & 0.4 & 0.4 & 0.2 & 0.1 & 0.3 \\
\hline & & & 2.1 & 2.4 & 2.2 & 1.6 & 2.0 & 3.0 & 0.2 & 0.3 & 0.4 & 0.2 & 1.2 & 0.4 & 2.4 \\
\hline & & & 1.8 & 1.5 & 1.3 & 1.9 & 1.3 & 1.8 & 1.6 & 1.7 & 1.4 & 1.7 & 2.6 & 1.7 & 1.7 \\
\hline \multirow[t]{3}{*}{$18 \mathrm{~A}$} & & & & 0.1 & 0.1 & 0.1 & 0.1 & 0.1 & 0.0 & 0.1 & 0.0 & 0.0 & 0.2 & 0.1 & 0.2 \\
\hline & & & & 3.7 & 4.1 & 2.1 & 2.4 & 1.7 & 3.0 & 2.9 & 3.0 & 2.6 & 1.5 & 1.6 & 2.0 \\
\hline & & & & 1.2 & 2.9 & 1.1 & 1.6 & 1.3 & 1.1 & 1.7 & 1.7 & 1.8 & 1.7 & 1.3 & 1.0 \\
\hline \multirow[t]{3}{*}{$19 \mathrm{~A}$} & & & & & 0.2 & 0.2 & 0.2 & 0.1 & 0.2 & 0.2 & 0.1 & 0.1 & 0.2 & 0.1 & 0.2 \\
\hline & & & & & 4.0 & 2.3 & 1.6 & 3.2 & 3.1 & 3.1 & 3.0 & 3.1 & 2.7 & 1.8 & 2.3 \\
\hline & & & & & 1.2 & 1.5 & 1.2 & 0.9 & 0.8 & 1.7 & 1.2 & 2.1 & 1.9 & 0.4 & 0.6 \\
\hline \multirow[t]{3}{*}{34} & & & & & & 0.1 & 0.1 & 0.1 & 0.1 & 0.1 & 0.1 & 0.1 & 0.2 & 0.1 & 0.2 \\
\hline & & & & & & 1.9 & 1.2 & 3.5 & 3.0 & 3.0 & 3.0 & 2.7 & 1.6 & 1.9 & 1.8 \\
\hline & & & & & & 1.6 & 1.1 & 2.1 & 0.7 & 1.3 & 1.5 & 1.7 & 2.0 & 0.8 & 0.5 \\
\hline \multirow[t]{3}{*}{35} & & & & & & & 0.2 & 0.1 & 0.1 & 0.1 & 0.1 & 0.1 & 0.2 & 0.0 & 0.1 \\
\hline & & & & & & & 1.1 & 4.0 & 1.0 & 1.0 & 1.0 & 0.8 & 1.6 & 0.7 & 2.1 \\
\hline & & & & & & & 1.5 & 0.7 & 1.0 & 1.0 & 2.0 & 0.9 & 1.7 & 1.2 & 0.7 \\
\hline \multirow[t]{3}{*}{ 34PS } & & & & & & & & 0.1 & 0.1 & 0.1 & 0.1 & 0.1 & 0.1 & 0.0 & 0.1 \\
\hline & & & & & & & & 2.6 & 2.5 & 2.4 & 2.5 & 1.9 & 2.1 & 1.0 & 1.3 \\
\hline & & & & & & & & 1.1 & 1.8 & 1.6 & 2.4 & 1.5 & 2.4 & 1.8 & 1.7 \\
\hline \multirow[t]{3}{*}{ 34PW } & & & & & & & & & 0.1 & 0.1 & 0.1 & 0.1 & 0.2 & 0.0 & 0.2 \\
\hline & & & & & & & & & 4.1 & 4.0 & 4.1 & 3.7 & 2.3 & 1.8 & 1.8 \\
\hline & & & & & & & & & 0.8 & 1.2 & 1.7 & 1.1 & 0.9 & 0.6 & 0.6 \\
\hline \multirow[t]{3}{*}{ 10P } & & & & & & & & & & 0.1 & 0.0 & 0.1 & 0.2 & 0.0 & 0.2 \\
\hline & & & & & & & & & & 0.7 & 0.6 & 0.8 & 1.6 & 0.5 & 2.4 \\
\hline & & & & & & & & & & 1.1 & 1.2 & 1.2 & 0.7 & 1.3 & 0.8 \\
\hline \multirow[t]{3}{*}{11} & & & & & & & & & & & 0.1 & 0.2 & 0.1 & 0.1 & 0.2 \\
\hline & & & & & & & & & & & 0.4 & 0.5 & 1.2 & 0.5 & 2.3 \\
\hline & & & & & & & & & & & 1.7 & 0.8 & 3.2 & 0.8 & 0.8 \\
\hline \multirow[t]{3}{*}{15} & & & & & & & & & & & & 0.1 & 0.2 & 0.1 & 0.2 \\
\hline & & & & & & & & & & & & 1.2 & 2.0 & 0.8 & 2.8 \\
\hline & & & & & & & & & & & & 1.9 & 1.6 & 1.7 & 0.9 \\
\hline \multirow[t]{3}{*}{17} & & & & & & & & & & & & & 0.3 & 0.1 & 0.2 \\
\hline & & & & & & & & & & & & & 1.2 & 0.5 & 2.2 \\
\hline & & & & & & & & & & & & & 3.1 & 1.3 & 0.7 \\
\hline \multirow[t]{3}{*}{$2 A$} & & & & & & & & & & & & & & 0.2 & 0.1 \\
\hline & & & & & & & & & & & & & & 1.5 & 1.8 \\
\hline & & & & & & & & & & & & & & 3.7 & 3.6 \\
\hline \multirow[t]{3}{*}{$58 \mathrm{P}$} & & & & & & & & & & & & & & & 0.2 \\
\hline & & & & & & & & & & & & & & & 2.4 \\
\hline & & & & & & & & & & & & & & & 1.2 \\
\hline
\end{tabular}



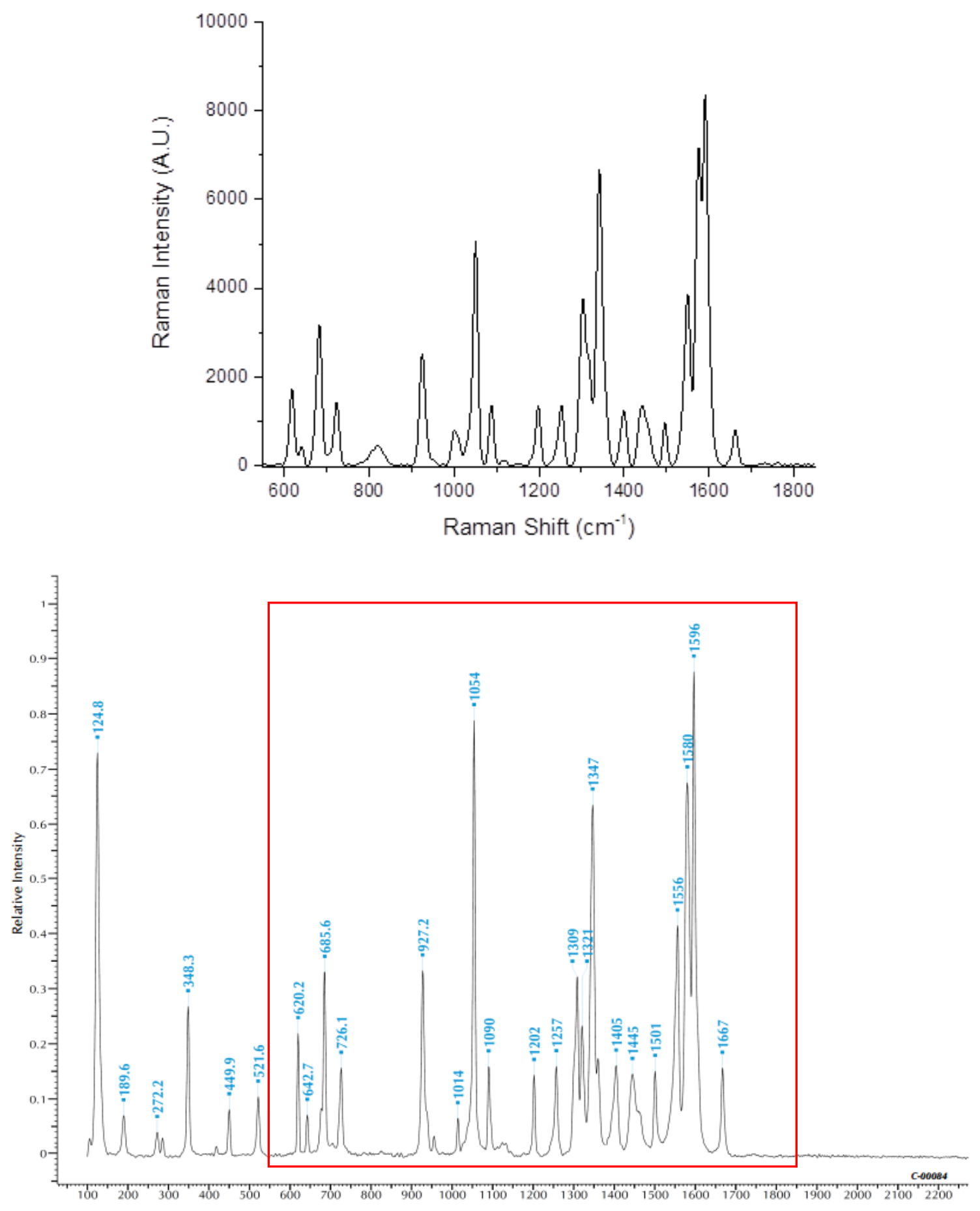

Figure A.1 Raman spectrum of the refinish base coat of sample 12 taken with the $785 \mathrm{~nm}$ laser (top), and a reference spectrum of PR254( $\alpha$ ) (bottom). Red box represents the spectral range of the sample spectrum. 

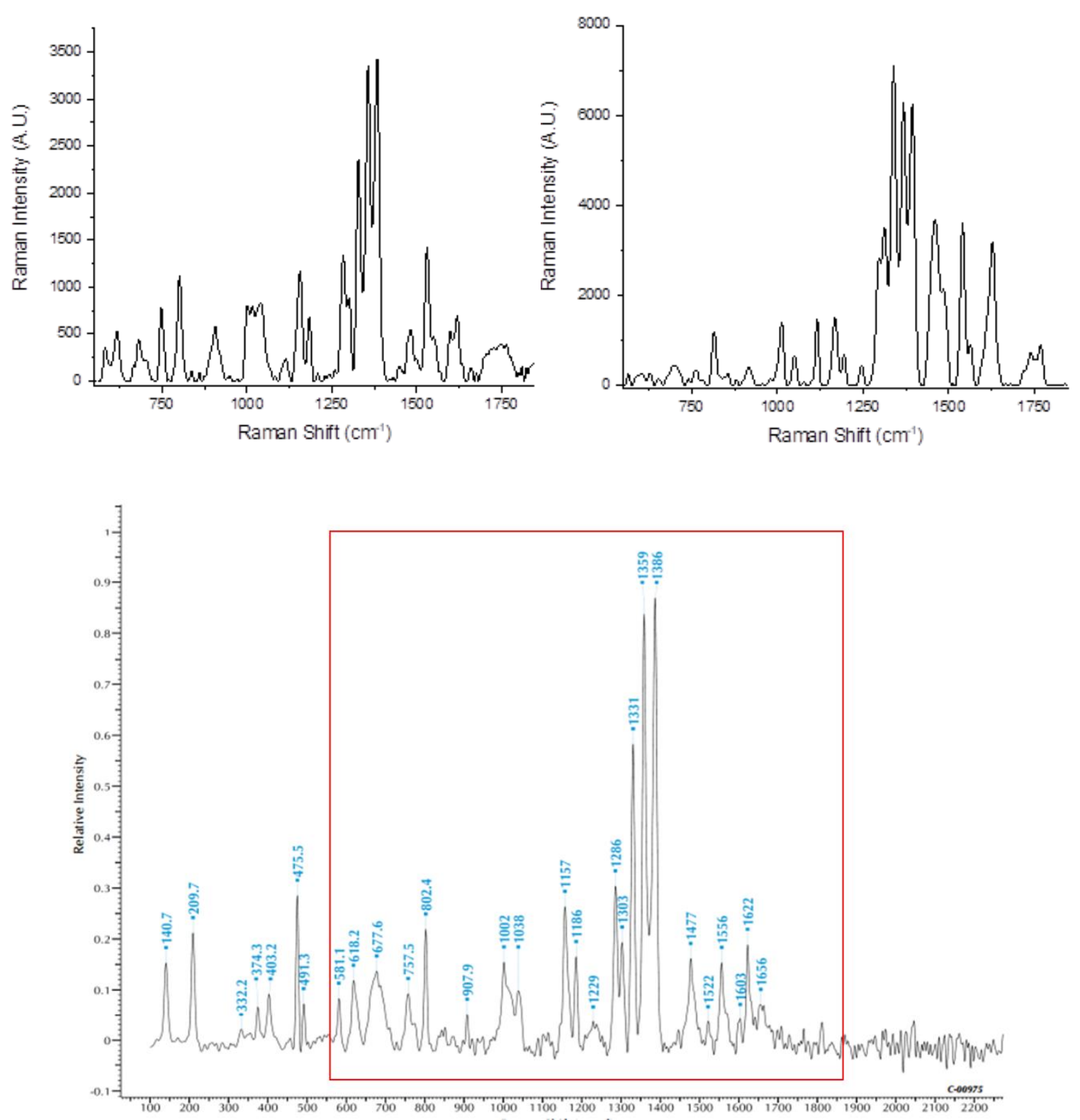

Figure A.2 Raman spectra of the original base coat of sample $18 \mathrm{~A}$ taken with the $785 \mathrm{~nm}$ laser (top left), the second effect layer of sample 1A taken with the $532 \mathrm{~nm}$ laser (top right), and the reference spectrum of PB60 (bottom). Red box represents the spectral range of the sample spectrum. 

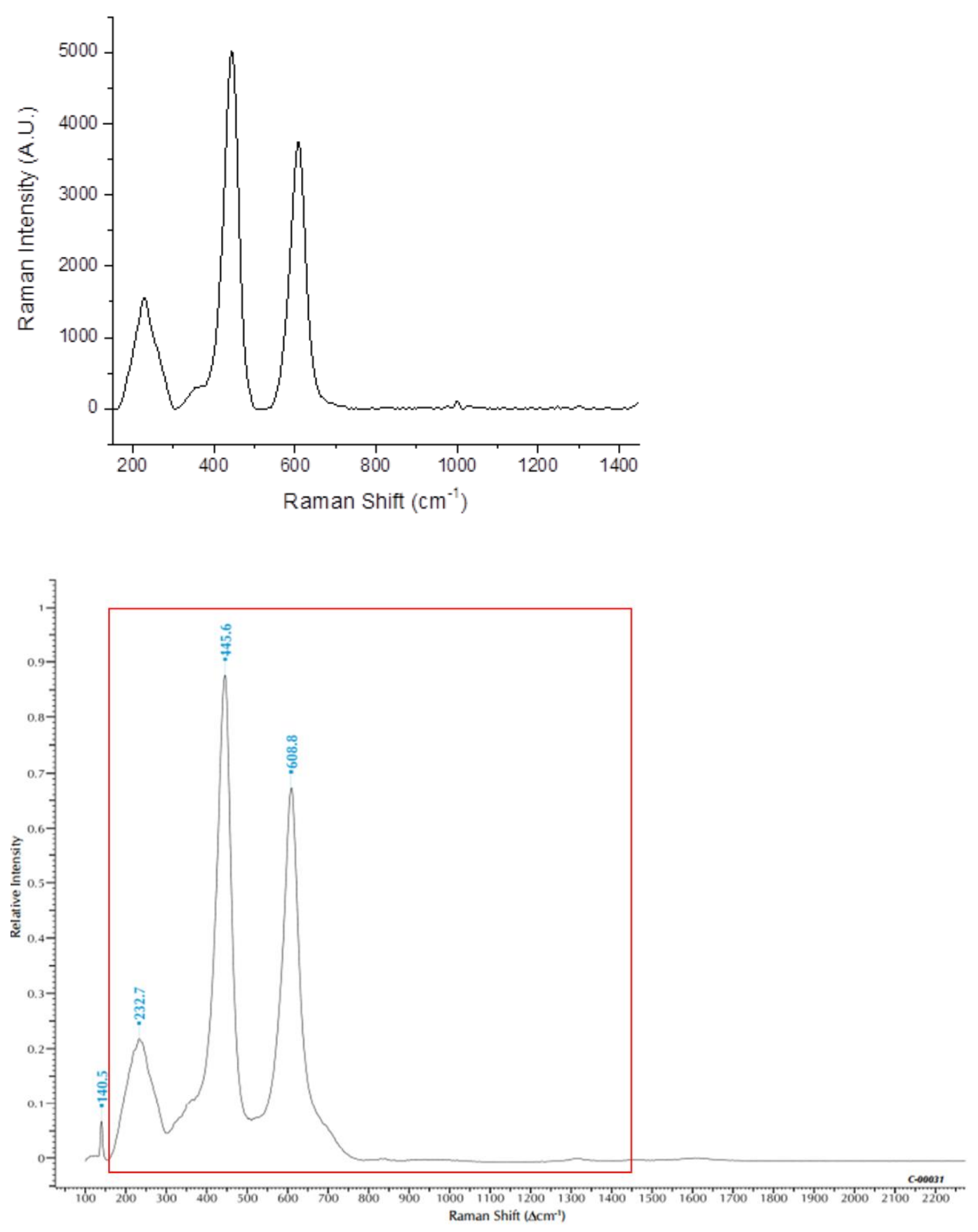

Figure A.3 Raman spectrum of the original base coat of sample 58 taken with the $785 \mathrm{~nm}$ laser (top), and a reference spectrum of PW6 (bottom). Red box represents the spectral range of the sample spectrum. 

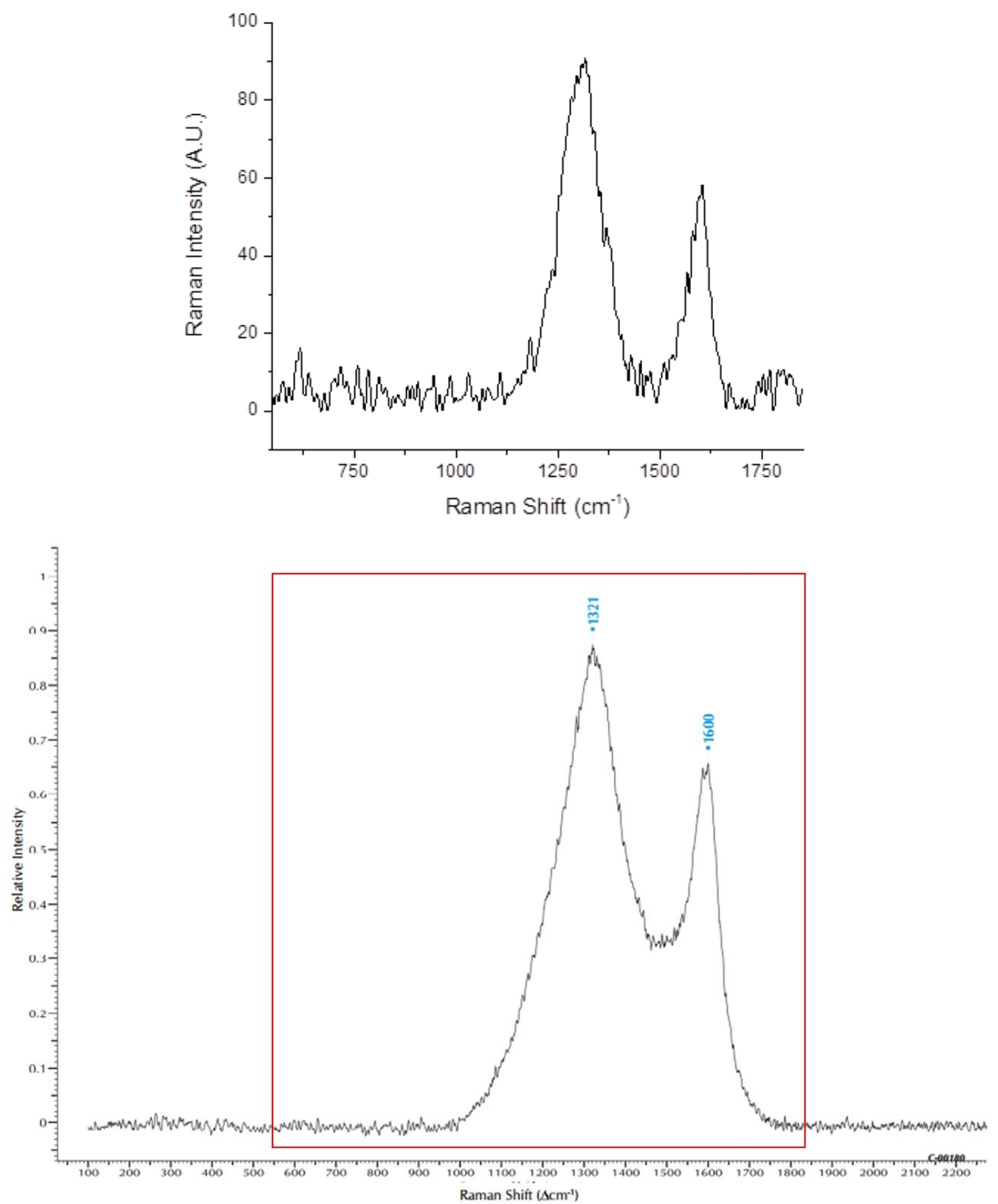

Figure A.4 Raman spectrum of the base coat of sample 10P taken with the 785 nm laser (top), and a reference spectrum of PBK7 (bottom). Red box represents the spectral range of the sample spectrum. 

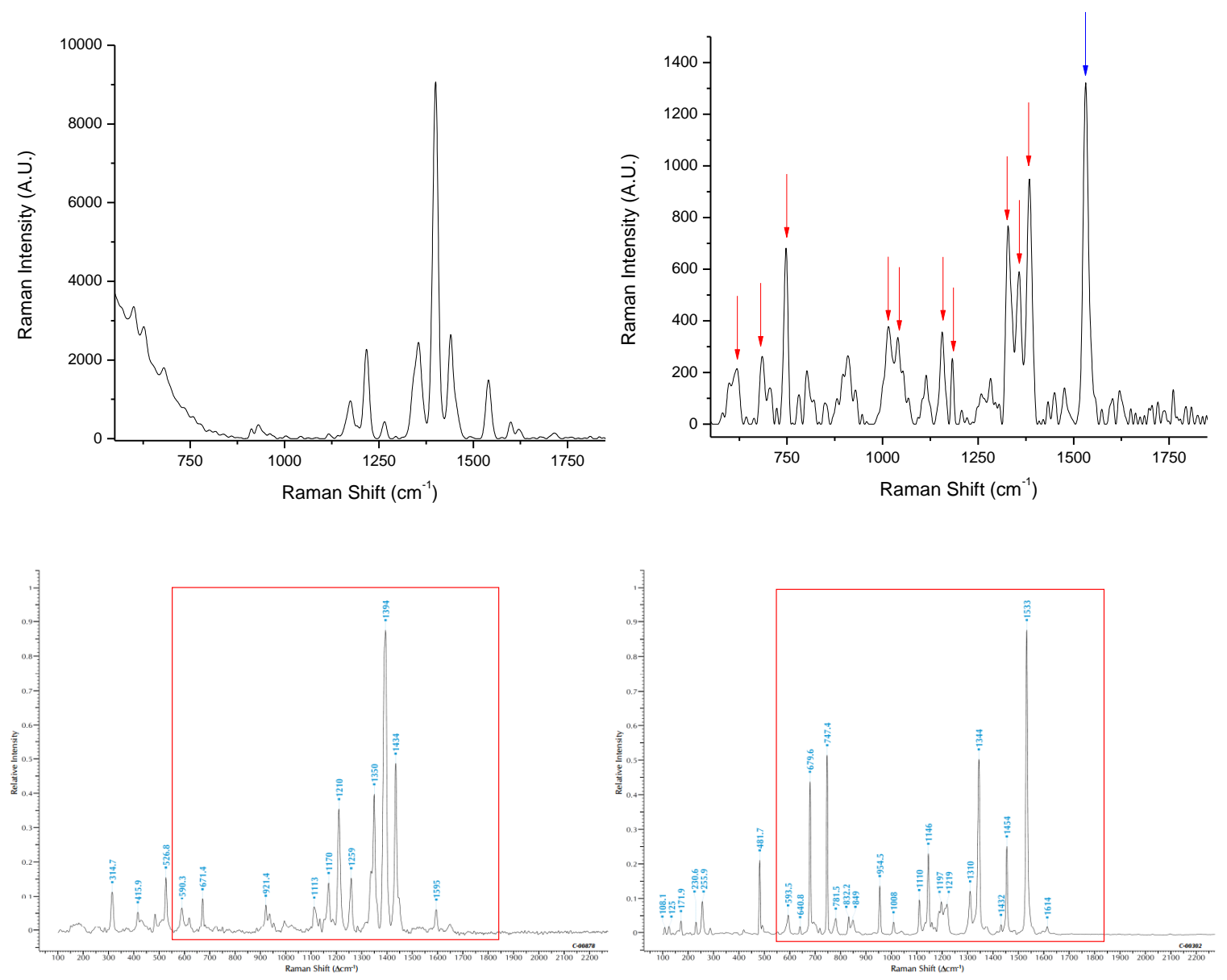

Figure A.5 Raman spectra of the base coat of sample 19A (top) taken with the 532 $\mathrm{nm}$ (left) and $785 \mathrm{~nm}$ (right) lasers, and reference spectra (bottom) of PV23( $\beta$ ) (left) and PB15 (right). Red box represents the spectral range of the sample spectrum. Red and blue arrows represent the peaks in the sample spectrum corresponding to PB60 and PB15, respectively. 

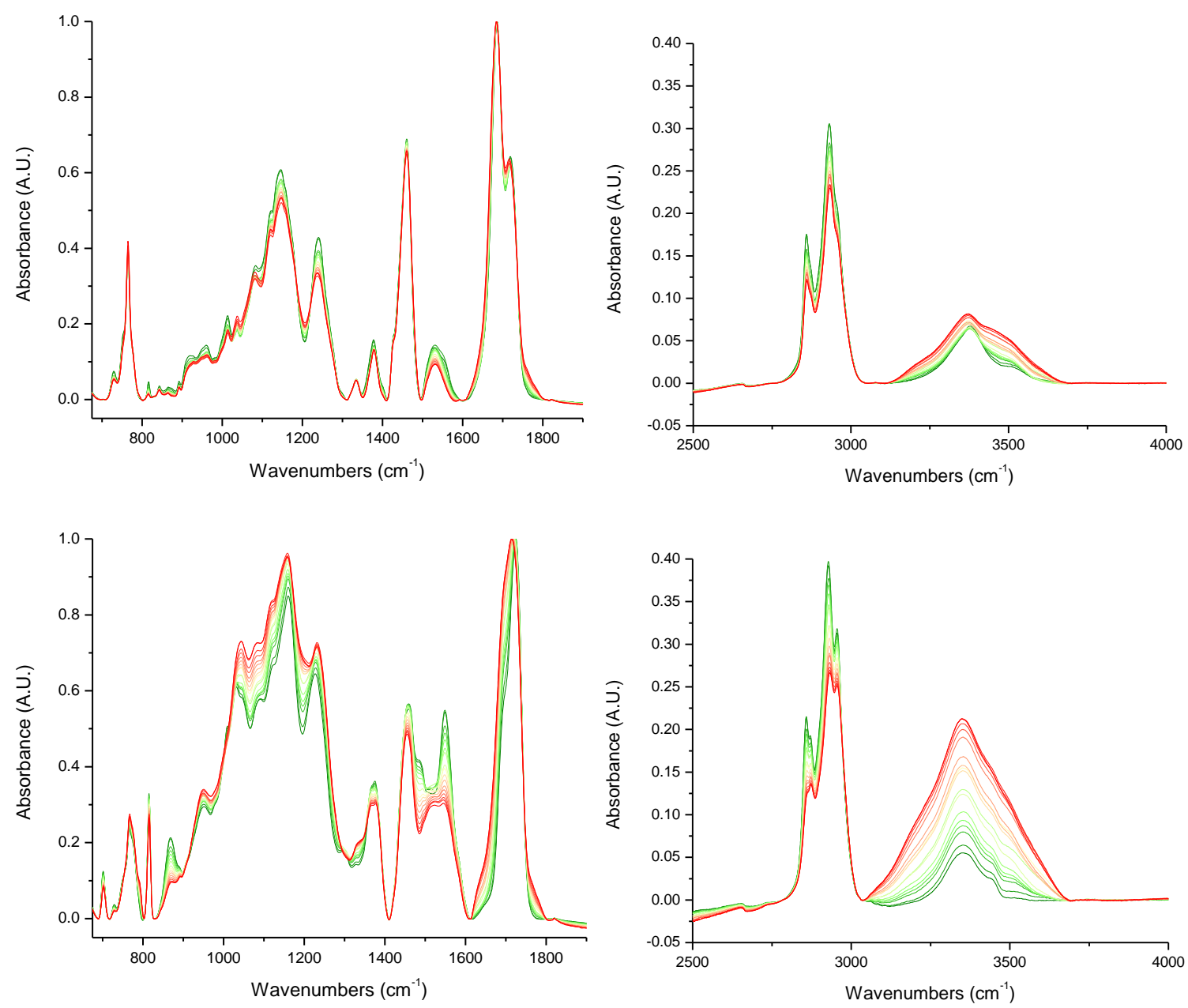

Figure A.6 ATR FTIR spectra of BASF E10CG081G (top) and R10CG392D (bottom) clear coats on black base coats collected with the iS50 instrument. Each spectrum represents a dose with increasing exposure from green to red. 

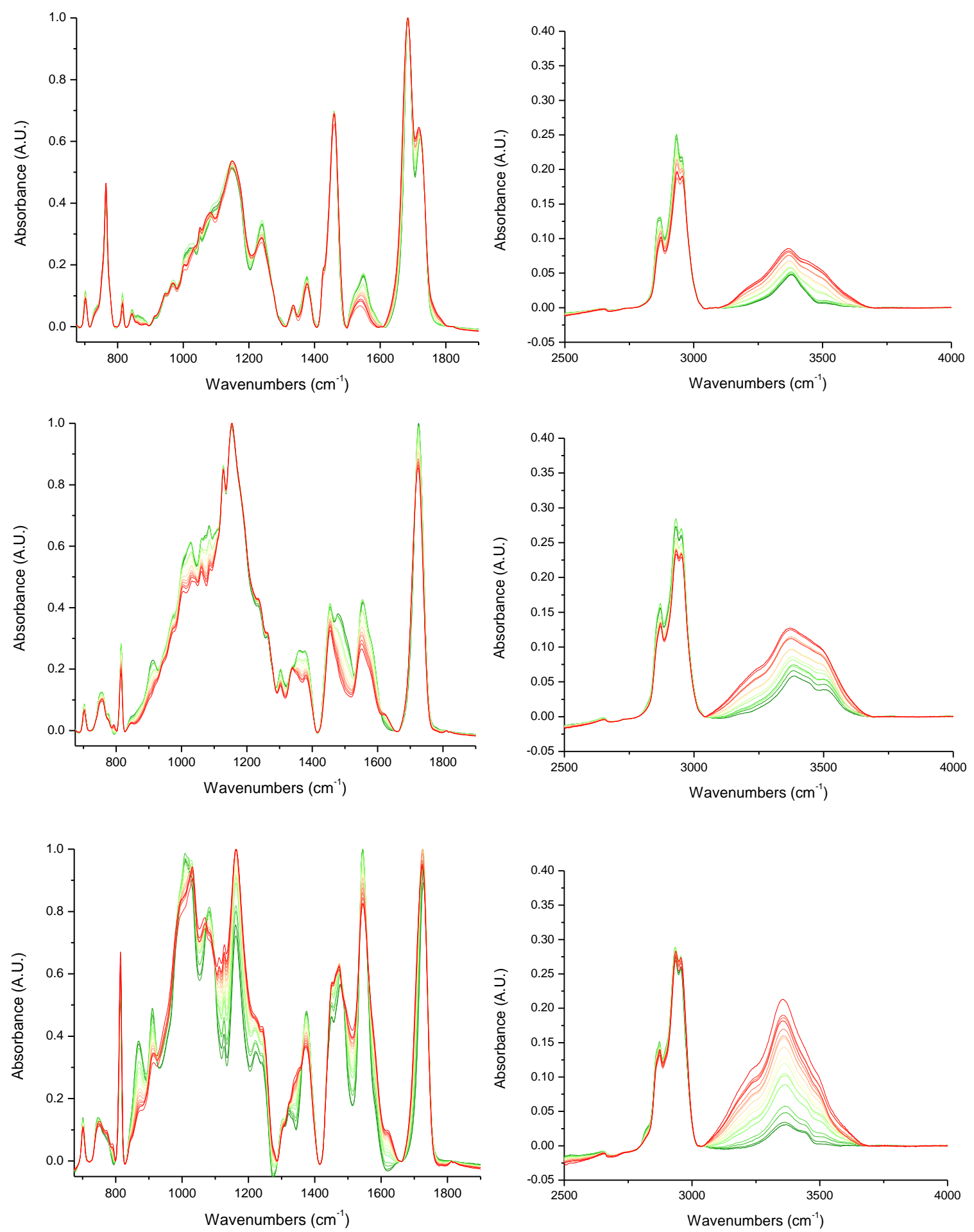

Figure A.7 ATR FTIR spectra of PPG (top to bottom) APO, NCT, and TMAC clear coats on black base coats collected with the iS50 instrument. Each spectrum represents a dose with increasing exposure from green to red. 